\title{
Nanoscale
}

Cite this: Nanoscale, 2013, 5, 7098

\section{Supramolecular self-assemblies as functional nanomaterials}

\author{
Eric Busseron, Yves Ruff, Emilie Moulin and Nicolas Giuseppone* \\ In this review, we survey the diversity of structures and functions which are encountered in advanced self- \\ assembled nanomaterials. We highlight their flourishing implementations in three active domains of \\ applications: biomedical sciences, information technologies, and environmental sciences. Our main \\ objective is to provide the reader with a concise and straightforward entry to this broad field by \\ selecting the most recent and important research articles, supported by some more comprehensive \\ reviews to introduce each topic. Overall, this compilation illustrates how, based on the rules of \\ supramolecular chemistry, the bottom-up approach to design functional objects at the nanoscale is \\ currently producing highly sophisticated materials oriented towards a growing number of applications \\ with high societal impact.
}

Received 30th April 2013

Accepted 24th May 2013

DOI: $10.1039 / c 3 n r 02176 a$

www.rsc.org/nanoscale could be the next challenges of this fascinating research area. We hope that this review will constitute a useful tool for the non-specialized readers who want to get an overview of the current trends related to self-assembled nanomaterials, or for experts who want to look for a precise entry in a particular domain of application.

\section{Self-assembled nanomaterials for biomedical applications}

Very recently, the physical and chemical properties of nanomaterials have inspired scientists to create new devices that are able to interact with biological systems at the nanoscale. While still in its early stages of development, the field of nanotechnology has already made a tremendous impact in medicine and biology in general. ${ }^{4,5}$ In particular, the field of nanobiotechnology - which refers to the use of biological systems and molecules to build nanoscale materials - is currently leading to promising applications in diagnostics and therapeutics. ${ }^{6,7}$ In this chapter we will illustrate the biomedical applications of various types of nanomaterials obtained by selfassembly (or which requires a self-assembly event with a substrate for producing the targeted function). In the first section, we will give a selection of the most recent advances in the field of diagnostics and sensing ${ }^{8,9}$ using self-assembled nanomaterials and including sensors based on optical, magnetic, and electrochemical detection. The second section will focus on recent examples of self-assembled nanomaterials for therapeutic applications. We will first highlight the use of nanomaterials for gene and drug delivery, ${ }^{9,10}$ in particular viruses, analogs of viruses, micellar systems, carbon nanotubes, inorganic/organic hybrid nanostructures, nanomaterials based on lipids or amphiphiles, and DNA. We will then describe
SAMS Research Group, University of Strasbourg, Institut Charles Sadron, CNRS, 23 rue du Loess, BP 84087, 67034 Strasbourg Cedex 2, France; Web: http://www-ics.u-strasbg. fr/sams.E-mail: giuseppone@unistra.fr 
recent applications of nanomaterials for regenerative medicine and tissue engineering. ${ }^{10}$

\subsection{Diagnostics and sensing}

Sensing of molecules for biomedical purposes requires the highest specificity and sensitivity to detect markers of diseases or active species such as drugs. Reduction of the cost and time of analysis is also an important consideration leading to increasingly miniaturized, non-destructive, and easy-to-read assays. In the following sections, we will illustrate the most recent advances in nanomaterials for biosensing applications, with detection modes based on optical, magnetic, or electrochemical assays.

\subsubsection{Optical sensors}

Gold nanoparticles. Among the various nanomaterials used for biosensing, nanoparticles are of high interest due to their inherent properties, i.e. size, high loading of signal tags, high surface area, and dynamic character for signal amplification. $^{11,12}$ A popular approach rests on the self-assembly of metals, and especially gold nanoparticles, ${ }^{13-16}$ decorated by ligands interacting with biomolecules. The optical properties of gold nanoparticles are ruled by their absorption at the surface plasmon resonance wavelength. This absorption is highly dependent on the chemistry taking place at the surface of the nanoparticles (e.g. in interaction with analytes) and on particleparticle interactions. ${ }^{17}$ For example, the aggregation of gold nanoparticles results in a visible color change from red to blue due to inter-particle coupling. ${ }^{13,15,17}$

Mirkin et al. pioneered this field with the introduction of single stranded DNA (ssDNA) decorated gold nanoparticles which can be used to detect extremely low concentrations of oligonucleotides through specific DNA sequence hybridization inducing aggregation. ${ }^{18}$ When $13 \mathrm{~nm}$ gold nanoparticles are functionalized with DNA sequences complementary to a target DNA molecule, base pairing induces aggregation of the particle, resulting in a visible color change due to interacting particle surface plasmons and aggregate scattering properties (Fig. 1a). These complexes also have extremely sharp melting transition temperatures allowing detection of even a single base pair mismatch of the complementary ssDNA. ${ }^{18}$ The highly selective linking of nanoparticles bearing complementary ssDNA sequences can even lead in some cases to highly ordered crystalline lattices where gold nanoparticles replace the atoms in traditional molecular crystals, following a new set of rules for the self-assembly of nano-objects. ${ }^{19}$

The self-assembly of nanoparticles mediated by biomolecules is not limited to nucleic acid detection and has also been extended to the sensing of proteins along the formation of antibody-antigen complexes. In particular, nanoparticle probes that are reversibly encoded with a DNA tag can reach a limit of detection for a cancer marker at a concentration as low as 30 attomolars. ${ }^{20}$ In this assay, nanoparticles decorated with antibodies and heavily functionalized with double stranded DNA barcodes are used to detect immunocomplexes formed by the prostate specific antigen (PSA) and PSA monoclonal antibody functionalized magnetic microparticles, after isolation of the complexes with a magnet and dehybridization of the DNA marker. Therefore a single binding event between an antibody and the target molecules translates into the release of multiple copies of the barcode DNA. Scanometric detection of this barcode can then be achieved at a concentration higher than that of the initial analyte. Indeed, an advantage of using DNA as a barcode is that further amplification of the signal can be achieved using polymerase chain reaction, effectively lowering the detection limit to 3 attomolar. ${ }^{20}$ Recently a similar bio-barcode assay was applied to the detection of amyloid diffusible ligands, which are biomarkers for Alzheimer's disease, in the cerebrospinal fluid of 30 patients at femtomolar (fM) concentration. ${ }^{21}$

The self-assembly of gold nanoparticles has also been used for the optical detection of enzymes because their activity can either promote or suppress the interparticle supramolecular interactions. ${ }^{22}$ For example, Stevens et al. have shown that protease activity can be detected through the breaking down of peptides that maintain gold nanoparticles in an aggregated state, resulting in a visible red shift of the sample color due to the dispersion of individual nanoparticles. ${ }^{23}$ The colorimetric detection of proteins was also achieved by Liedberg et al. using specific interactions between a model protein, the human carbonic anhydrase, and a synthetic receptor present at the surface of gold nanoparticles to hinder their aggregation by selfassembly. ${ }^{24} \mathrm{~A}$ similar strategy using the interaction of a peptide derived from a viral protein and an antibody to prevent the aggregation of gold nanoparticles allowed the detection of the viral peptide with a detection limit of $10 \mathrm{nM} .^{24}$

The reverse approach using aggregation of gold nanoparticles to detect enzymatic activity was reported by Stevens et al., who used the immuno-aggregation of nanoparticles after kinase mediated phosphorylation of tyrosine residues in peptides. ${ }^{25}$ Indirect detection of enzyme activity has also been reported..$^{26}$ In this system, treatment of liposomes with phospholipase triggers the release of a peptide specifically designed to recognize a second polypeptide immobilized on gold nanoparticles. Again, this event results in a visible color change due to the aggregation of nanoparticles. ${ }^{26}$

As an alternative to colorimetric assays, the detection of cancer biomarkers using dynamic light scattering has been proposed by Huo et al..$^{27}$ with the advantages that the reaction takes place directly in solution and that it requires smaller amount of samples. The quantitative determination by DLS of the ratio between antibody decorated gold nanoparticles and larger immunocomplexes obtained after addition of the prostate specific antigen (PSA) allows the detection of the antigen at very low concentrations $\left(0.5 \mathrm{ng} \mathrm{ml}^{-1}\right) .^{27}$

Besides assays that are based on the self-assembly of nanoparticles, Rotello et al. have used the reversible character of supramolecular complexes between charged nanoparticles and oppositely charged fluorescent conjugated polymers to elaborate "chemical noses" for the detection and identification of proteins (Fig. 1b). ${ }^{28,29}$ The principle here consists in mixing a protein of interest separately with six different gold nanoparticles/conjugated polymer complexes, and in recording on a plate reader the individual fluorescence of each of the six samples. The polymers that have been displaced by the 

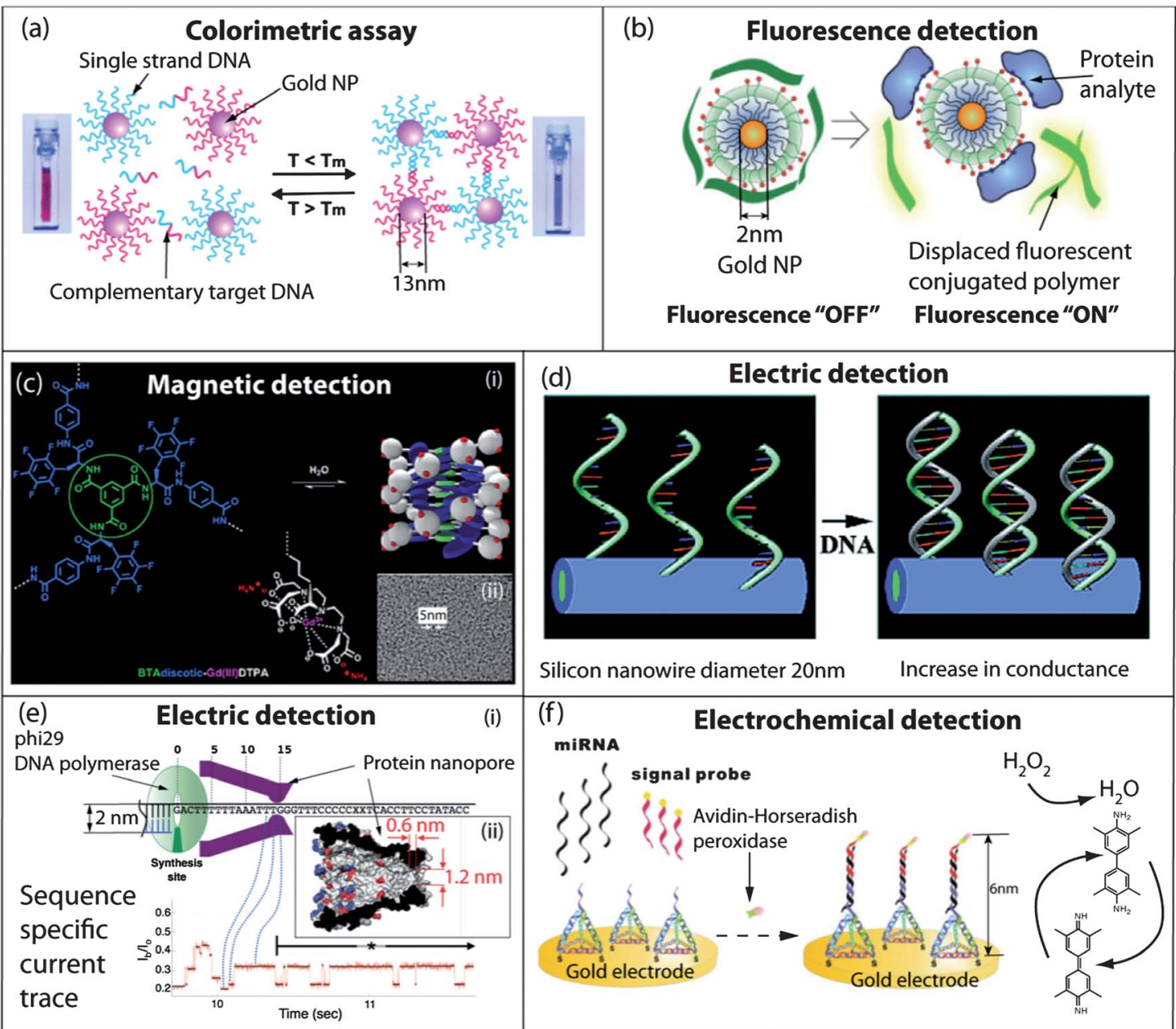

Fig. 1 Self-assembled nanomaterials for diagnostics and sensing. (a) Colorimetric detection of single stranded DNA by hybridization with gold nanoparticles decorated with complementary single stranded DNA. The resulting aggregates, which display very sharp melting transitions, are easily observed by a visible color change or by UV-Vis spectroscopy. (b) Fluorescence detection of proteins by displacement of quenched conjugated polymers bound at the surface of a gold nanoparticle. The extent of the polymer release in solution and the resulting fluorescence are dependent on the nature of the interaction occurring between the protein and the cationic and hydrophobic groups in the nanoparticles' corona. (c) (i) Self-assembly of a fluorinated benzene-1,3,5-tricarboxamide in well-defined water soluble paramagnetic nanostructures as efficient MRI contrast agents; (ii) cryo-TEM micrograph of the self-assembled amphiphile in a serum albumin PBS buffered solution. (d) Conductance increase of silicon nanowires decorated with ssDNA upon hybridization with complementary DNA fragments. (e) (i) Self-assembled MspA protein nanopore used for the sequencing of single DNA molecules; (ii) crystal structure of the MspA protein. Reading of the sequence of the DNA template is achieved through the change in ionic current upon the translocation of the nucleobases through the pore, across a membrane separating two compartments. (f) Ultrasensitive electrochemical assay for the detection of miRNA using a self-assembled DNA tetrahedron for the spatial and accessibility control of an electrochemical probe, horseradish peroxidase. Panel (a): reproduced with permission from ref. 14; panel (b): reproduced with permission from ref. 28; panel (c): reproduced with permission from ref. 41; panel (d): reproduced with permission from ref. 47; panel (e): reproduced with permission from ref. 58; panel (f): reproduced with permission from ref. 65.

electrostatic interactions between the protein and the charged nanoparticles are responsible for the increase of fluorescence. As for the six different complexes the dissociation occurs to different extents and the resulting fluorescence pattern is specific to each protein. This assay was successfully used to identify 52 unknown protein samples with an accuracy of $94.2 \% .^{28}$

Supramolecular gels. As for enzyme-responsive nanoparticle aggregation-dissociation assays, enzyme triggered assemblydisassembly of small molecules can also provide a readout for the sensing of glucose oxidase ${ }^{30}$ or proteases. ${ }^{31}$ This can be achieved through the formation of a macroscopic gel using a peptide precursor containing cysteine residues and 2-cyanobenzothiazole units. This gelation occurs by the condensation of these two functions after treatment with a protease or by disulfide reduction. The gel formation can take place in vitro but also inside living cells, ${ }^{31}$ enabling for instance the imaging of the proteolytic activity of furin. A similar system was used by Liang et al. to design a sensor for caspase-3 based on the fluorescence enhancement of fluorescein-streptavidin conjugates upon mixing with self-assembled biotinylated nanospheres. ${ }^{32}$ Sensing of enzymatic activity can also be achieved indirectly upon a $\mathrm{pH}$ change induced by glucose oxidase, resulting in the assembly or disassembly of $\mathrm{pH}$-sensitive peptide amphiphiles. ${ }^{30}$ Arrays. The adaptative change in optical properties of dynamic self-assembled small molecules upon the introduction 
of an analyte can also be used for detection. Arrays of selfassembled complexes have been used in colorimetric assays for the detection of carbohydrates. ${ }^{33}$ Reversible covalent bonds between carbohydrates and boronic acids allow for the dynamic rearrangement of boronic esters in carbohydrate-chromophore conjugates. For example, the addition of a polyol indicator to a library of solid-supported boronic esters of carbohydrates results in the displacement of the sugar. The rate and extent of the displacement are functions of the stability of the carbohydrate boronic ester and colorimetric arrays can be obtained to identify specific carbohydrates. ${ }^{34}$ More examples of arrays based on dynamic supramolecular complexes can be found in the following section on nanomaterials for environmental sciences.

DNA nanostructures. Self-assembled DNA nanostructures loaded with intercalator dyes have also been used as nanotags with potential use for tracking and labelling biomolecules (proteins, antibodies, nucleic acids, etc.). Indeed, when chromophores are protected and concentrated within a small volume, they display enhanced resistance to nuclease digestion and improved photostability. ${ }^{35}$ Using this approach, the coassembly of donor and acceptor dyes has led to efficient energy transfer systems with tunable emission wavelength. ${ }^{35}$ More recently Shih et al. have described fluorescent barcodes obtained by the positioning of fluorophores on the surface of a stiff self-assembled DNA nanorod or on non-linear nanostructures. ${ }^{36}$ These nanoscale barcodes are observable by epifluorescence or total internal reflection fluorescence microscopy and can be used to tag yeast surface receptors, which suggests their potential applications as in situ imaging probes. ${ }^{36}$ Self-assembled molecular logic gates based on DNA hybridization can also be used for the optical detection of M. tuberculosis DNA. ${ }^{37}$ More examples of DNA-based logic gates are described in the following section on nanomaterials for information technology.

\subsubsection{Magnetic sensors}

Iron nanoparticles. The formation of large supramolecular aggregates of super-paramagnetic iron oxide nanoparticles has a direct effect on the spin-spin relaxation time of billions of neighboring water molecules, resulting in signal amplification. ${ }^{38}$ Immuno-aggregation of super-paramagnetic iron oxide nanoparticles, decorated with specific antibodies, and multivalent bacteria or cancer cell surfaces resulted in the formation of $300 \mathrm{~nm}$ clusters of magnetic nanoparticles that can be used in miniaturized NMR assays to detect bacteria or cancer markers in biological fluids. ${ }^{39}$

Self-assembling paramagnetic gadolinium complexes. The incorporation of paramagnetic metal complexes into selfassembled structures is also of great interest as it allows the preparation of NMR contrast agents with well-defined morphology and dimensions. ${ }^{\mathbf{4 0 4 1}}$ This strategy combines the benefits from low molecular weight imaging agents with the ones of macromolecular contrast agents, as self-assembled nanostructures are degradable through their ability to disassemble into their individual components, while still showing enhanced relaxivity in their assembled states. Specifically, selfassembled dendritic-like nanoparticles have been prepared by the self-assembly of a $C_{3}$-symmetrical benzene-1,3,5- tricarboxamide (BTA) core that directs the self-assembly into triple hydrogen-bonded helices decorated by Gd(III)-DTPA (diethylenetriaminepentaacetic acid) chelates (Fig. 1c). ${ }^{40} \mathrm{~A}$ high degree of control on the size and shape of these supramolecular polymers was achieved in water by tuning the electrostatic repulsion between the monomers. ${ }^{40}$ The stability and potency for contrast-enhanced MRI have been demonstrated both in vivo and in vitro. ${ }^{41}$ Controlled intracellular self-assembly of paramagnetic gadolinium complexes has also been described recently by Liang et al. In this system, the condensation between 2-cyanobenzothiazole and N-terminal cysteine has been used to detect the degradation of paramagnetic peptidic precursors by furin, a protease overexpressed in tumors. The increase in relaxivity upon condensation and subsequent self-assembly were used to detect tumors in vivo (mouse model). ${ }^{42}$

\subsubsection{Electrochemical and amperometric sensors}

Gold nanoparticles. Mirkin et al. have developed an elegant process to take advantage of the selective hybridization of a target DNA with complementary ssDNAs grafted both on a surface between electrodes and on the surface of gold nanoparticles. This leads to the localization of the gold nanoparticles between electrodes which facilitates silver deposition, thus resulting in a measurable increase in conductivity. Target DNA concentrations as low as $500 \mathrm{fM}$ were detected with a point mutation selectivity factor of $100000: 1^{43}$

Semiconducting nanowires. The small diameter of the nanowires allows a large change in conductance upon binding of charged biomolecules at their surface. This strong effect rests on the charge carrier depletion/accumulation within the entire diameter of the wire. ${ }^{\mathbf{1 2}}$ Therefore, field-effect transistors based on such nanostructures have been extensively used for the detection of biomolecules. ${ }^{4-46}$ Lieber et al. pioneered the use of semiconducting silicon nanowires for the design of DNA sensors. They took advantage of the change of conductivity upon binding of the negatively charged SsDNA to silicon nanowires decorated with neutral peptide nucleic acid sequences (Fig. 1d). ${ }^{47}$ Concentration of ssDNA as low as $10 \mathrm{fM}$ can be detected. More recently similar devices have been used for the detection of ssDNA under physiological buffer and salt concentration conditions. ${ }^{48}$

Carbon nanotubes. The conducting properties and high aspect ratio of carbon nanotubes have been used to design fieldeffect transistors for the sensing of biomolecules, in particular DNA, antibody-antigen complexes, and sugars. ${ }^{49,50}$ The selfassembly of the carbon nanotubes themselves represents another strategy to build electrochemical sensors. ${ }^{51}$ The selective single-walled carbon nanotubes (SWNTs) assembly onto 16mercaptohexadecanoic acid self-assembled monolayers deposited on gold electrodes facilitates electron transfer between the electroactive species and the electrode. Sensing was achieved through the binding of the protein of interest (a tumor biomarker folate receptor) with folate molecules attached to the extremity of ssDNA. This binding prevents the degradation of the DNA strand coated carbon nanotube by the nuclease Exo1. Therefore in the presence of the protein of interest, the coated nanotube does not interact with the working surface of the gold electrode. In the absence of the supramolecular protection 
provided by the protein, the ssDNA coating of the nanotube is degraded by Exo1 resulting in the absorption of the SWNT on the working surface of the electrode. A detection limit of $3 \mathrm{pM}$ in the folate receptor was reached using this quantitative analysis for its interaction with folate. ${ }^{51}$

Self-assembled peptide nanowires. We have seen above that electrochemical activity of electrodes can be modified by the adsorption of nanostructures like carbon nanotubes on their surfaces. The nanostructures themselves can be obtained by self-assembly. For example, Gazit et al. deposited dense arrays of well-ordered self-assembled peptide nanotubes or nanospheres, obtained from Boc-protected phenylalanine dipeptides, at the surface of gold electrodes, and characterized by chronoamperometry. ${ }^{52}$ A 17 -fold improvement in the sensing performance for phenol, with respect to the uncoated electrode, was observed. More recently, a conducting peptide/polypyrrole making use of vertically aligned diphenylalanine peptide nanowires was obtained by chemical polymerization and characterized by cyclic voltammetry. ${ }^{53}$ The resulting peptide/polypyrrole coated electrodes were used as dopamine sensors with a detection limit of $3.1 \mu \mathrm{M}$. Interestingly, cell growth experiments proved that the modified electrodes are suitable as cell culturing platform materials.

Self-assembled nanochannels. Self-assembled protein channels with nanometric dimensions are often found in nature to control the transport of ions or molecules across membranes. As crystallisable self-assembled proteins, the structures of such nanopores can be determined with atomic precision in some cases, and can also be expressed, modified, and optimized by genetic engineering, making them ideal candidates for reproducible use in sensing devices. In particular, the application of protein or solid-state nanopores as electrosensors has a tremendous potential to lower the cost of sequencing technologies. ${ }^{\mathbf{5 4 5 5}}$ Indeed, if a voltage is applied between two compartments separated by a lipid bilayer, and permeated only by a single pore, the residual ionic current is going to be affected by the electrophoretic transport of molecules, or macromolecules, through the nanopore. For example, the channel created by $\alpha$-hemolysin incorporated in a lipid bilayer is $2.6 \mathrm{~nm}$ wide, $5 \mathrm{~nm}$ long, and with a $1.4 \mathrm{~nm}$ bottleneck which means that ssDNA can pass through the nanopore while the double-stranded one cannot. This allows the threading of single ssDNA molecules through the pore. At any given time, the residual ionic current will be dependent on which nucleotide is passing through the pore. The covalent attachment through the disulfide bond of a cyclodextrin into the cavity of $\alpha$-hemolysin allows for even better discrimination between molecules transported across the pores and to identify nucleosides with $99.8 \%$ accuracy. ${ }^{56}$ In principle, it is therefore possible to determine the sequences of bases of the ssDNA molecule. However, under electrophoretic conditions, ssDNA moves through the nanopores at very high velocities (one nucleotide per $\mu \mathrm{s}$ ), which make correct identification of a given nucleotide difficult. ${ }^{54,55}$ Recently, two groups circumvented these limitations by slowing down the translocation of the ssDNA with enzymatic motors responsible for DNA replication. Akeson et al. described the forward and reverse ratcheting of DNA templates through hemolysin at 2.5-40 nucleotides per second. ${ }^{57}$ Their approach consisted in complexing a DNA template bearing a blocking oligomer with phi29 DNA polymerase (DNAP). A similar approach was independently reported by Gundlach et al. using DNAP to pull the ssDNA template through Mycobacterium smegmatis porin A (MspA) (Fig. 1e). ${ }^{58}$ They demonstrated that the residual current levels are related to the sequence of the individual ssDNA traversing the MspA nanopore. These impressive achievements demonstrate the preparation of nanoscale self-assembled sensors incorporating molecular motors to manipulate and control the translocation of ssDNA through a self-assembled nanoscale reading unit.

DNA-based nanostructures. The self-assembly properties of DNA have nowadays reached a level of refinement allowing the predictive self-assembly of $2 \mathrm{D}$ or $3 \mathrm{D}$ hierarchical structures with a high level of complexity. ${ }^{59-62}$ In particular, the ability to use the DNA scaffold to precisely position different molecular components in three dimensions can be used to build electrochemical sensing devices. ${ }^{\mathbf{6 3 , 6 4}}$

Fan et al. have described the use of the self-assembled DNA tetrahedron to engineer the working surface of gold electrodes for ultrasensitive detection of miRNA. ${ }^{65}$ In this example, the hybridization of the target miRNA acts like a linker between the rigid DNA tetrahedron spacer and an electrochemical probe, namely the avidin-horse radish peroxidase conjugate (avidinHRP). This results in the localization of the probe at a certain distance from the electrode surface, avoiding the crowding effect and improving mass transport at the water-electrode interface (Fig. 1f). The authors have demonstrated enhanced binding recognition at the gold electrode surface and drastically improved detection sensitivity up to attomolar amounts of miRNA with single base discrimination ability. Linear constructs involving the formation of sandwich-type complexes of DNA molecules to introduce a spacer between the working electrode and avidin-HRP were also described, allowing for a detection limit of 10 copies of the target DNA strand. ${ }^{66}$

The DNA scaffold itself can also serve as self-assembled binding pockets for the detection of analytes. For example, aptamers have been used to build electrochemical sensing devices for small hydrophobic molecules of biological interest like drugs. ${ }^{67,68}$

\subsection{Therapy and delivery}

It is clear that recent progress in nanotechnology has deeply impacted the field of drug delivery. ${ }^{69}$ In particular, self-assembled nanostructures are highly valuable for therapeutic and delivery applications. Their size, high loading capacity, and reversible character are major assets for the controlled release of therapeutics. In addition, biomolecules and biopolymers can be efficiently used to design such nanostructured materials that are of high interest to confer them on biocompatibility and

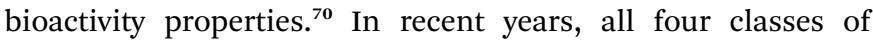
biomolecules (peptides, ${ }^{70-73}$ lipids, ${ }^{74-76}$ nucleic acids ${ }^{59,77-80}$ and sugars $^{\mathbf{8 1 - 8 5}}$ ) have been studied as building blocks for selfassembled nanostructures, yielding increasingly complex and refined therapeutic agents and nanoscale vectors. 


\subsubsection{Gene and drug delivery}

Viruses. The archetype of a highly efficient nanoscale delivery agent is a virus, as its life cycle requires specific mechanisms for cell targeting, endocytosis, and hijacking of the cellular machinery through the intracellular release of the viral genome. These organisms have been optimized by billions of years of Darwinian evolution, yielding the most efficient vectors of nucleic acid into living organisms. Their diameters are in the range of 25-30 nm for icosahedral viruses, and in the range of 6-20 $\mathrm{nm}$ in diameter and 300-900 $\mathrm{nm}$ in length for filamentous viruses. ${ }^{86}$ If native viruses aim at carrying the viral genome into healthy cells for ensuring their reproductive cycle, the genetic manipulation of these natural viruses such as lentiviruses ${ }^{87}$ has led to human therapies ${ }^{88}$ for cancer or HIV (Fig. 2a). ${ }^{89}$

Viruses can also be inactivated by the removal of their genetic information. Viral capsid proteins can be expressed in bacteria, isolated and self-assembled into homogeneous nanocarriers that have been used to develop novel drug and gene delivery agents. ${ }^{86}$ The viral capsids, self-assembled with or without a template, can be used to encapsulate enzymes, nucleic acids, or negatively charged polymers. ${ }^{90}$ Viral proteins can also be modified chemically by protein engineering and possibly by incorporation of unnatural aminoacids, to confer novel properties to these nanocontainers. ${ }^{86}$ Covalent modification of viruses with polyethylene glycol (PEG) residues and with targeting ligands for site-specific delivery is of particular interest for in vivo therapeutic applications. ${ }^{91}$ Viral particles are therefore a modular platform yielding precisely defined selfassembled icosahedral or filamentous nanoparticles that can be chemically tailored for specific biomedical applications. ${ }^{92}$

Self-assembled virus-like structures. Viral particles have several drawbacks such as their pathogenic and immunogenic properties. Expression of viruses or viral proteins also requires specialized techniques and synthetic analogs accessible by the traditional tools of organic synthesis are of high interest. What makes a nanostructure so-called "virus-like" is subject to interpretation, but very elegant approaches have been described to create self-assembled nanostructures with dimensions and architectures reminiscent of viruses..$^{\mathbf{9 3}, 94}$ The highly complex icosahedral viral capsids are in reality composed of a limited number of $C_{3}$-symmetric ${ }^{95}$ hexagonal or pentagonal protein building blocks. ${ }^{96}$ The first rational design of a $C_{3}$-symmetric self-assembling $\beta$-sheet peptide to mimic the self-assembly of spherical viruses has led to the preparation of trigonal-(FKFE) peptides self-assembling into $20 \mathrm{~nm}$ nanospheres through antiparallel $\beta$-sheet formation. ${ }^{95}$ More recently, the same group reported the self-assembly of "tailor-made" hollow 30-50 nm virus-like nanocapsules, from 24-mer peptides able to selfassemble into a $C_{3}$-symmetric $\beta$-annulus structure. ${ }^{97}$ In another study, regular polyhedral peptidic nanoparticles with a diameter of $16 \mathrm{~nm}$ were prepared by covalently linking coiled-coil peptides with $C_{3}$ - and $C_{5}$-symmetry and applied to immunization experiments in a mouse model (Fig. 2b). ${ }^{\mathbf{9 8}, 99}$ Homogeneous virus-like micelles with a diameter of $20 \mathrm{~nm}$ were also prepared from the self-assembly of coiled-coil lipopeptides. ${ }^{100}$ The design of this peptide was optimized for the display of multiple copies of an antigen and used for the adjuvant-free stimulation of antigen-specific humoral immune responses for potential applications in vaccine development. ${ }^{\mathbf{1 0 0}}$

Along their continuous efforts to build functional nanocages from synthetic organic ligands and metal ions (see Section 4.4), Fujita et al. have described, for the first time, the encapsulation of a native protein into a giant self-assembled nanocage with a diameter of $6.3 \mathrm{~nm}$ (Fig. 2c).${ }^{101}$ When compared with viruses, these nanocages are smaller and lack several of their key functions. However, one can envision that, by an optimized design of the organic ligands, modifications will take place within or outside the nanocage in order to allow encapsulation of nucleic acid or targeted delivery.

To go one step further and design particles capable of binding nucleic acids, synthetic functional analogs of filamentous viruses have been developed by Lee et al. who designed $\beta$-sheet peptides which self-assemble into nanoribbons and form filamentous complexes with siRNA (Fig. 3a). ${ }^{\mathbf{1 0 2}}$ These structures can be loaded with a hydrophobic dye and decorated with sugars for enhanced endocytosis in cancer cells. Another original strategy for the self-assembly of synthetic glycoviruses was described by Aoyama. ${ }^{103}$ Here, macrocyclic calixarene scaffolds were selectively decorated with sugars on one side and a hydrophobic tail on the other. These amphiphiles self-assemble irreversibly in $5 \mathrm{~nm}$ micelles, which can then be agglutinated by phosphate ions. The authors used this affinity for phosphate ions to complex plasmid DNA and obtained virus-like particles with a diameter of $50 \mathrm{~nm}$ that were able to achieve high transfection efficiency in vitro. ${ }^{103}$

Liposomes. Liposomes are closed spherical lipid bilayers obtained by the aqueous self-assembly of lipids. Their loading by therapeutic agents can be achieved by physical entrapment into the intra-liposomal aqueous phase or by diffusion within the hydrophobic section of the lipid bilayers. They were first described in 1965 (ref. 104) and are still under active clinical development today. Due to their ease of preparation, their fine tunable properties, and their functionalization through the coassembly of different lipids, liposomes are amongst the most common self-assembled nanoscale vectors for a wide range of therapeutic agents such as drugs or nucleic acids. ${ }^{\mathbf{1 0 5 - 1 0 7}}$ Liposomal delivery systems have been the first self-assembled nanomaterial approved for human clinical treatment against cancer in particular. ${ }^{\mathbf{1 0 8 , 1 0 9}}$ For example, Doxil ${ }^{\circledR}$ was approved in 2007 by the FDA and consists of a formulation of the anticancer drug doxorubicin in PEGylated "stealth" liposomes with a diameter around $100 \mathrm{~nm} .^{\mathbf{1 1 0}}$

Lipoplexes. While most lipids used for liposomal drug delivery are anionic or neutral, e.g. phosphatidylcholine, the use of cationic lipids is of particular interest for the delivery of nucleic acids by liposomes, namely lipofection. ${ }^{\mathbf{1 1 1}-\mathbf{1 1 4}}$ The principle behind the design of these vectors is simple as it aims to take advantage of electrostatic interactions between cationic lipids and negatively charged therapeutic agents that are nucleic acids. Strong electrostatic interactions between nucleic acids and the cationic head of lipids yield complexes, i.e. lipolexes, having a wide range of architectures and liquid crystalline phases. ${ }^{\mathbf{1 1 5 - 1 1 7}}$ The bioactivity of these various lipoplex 

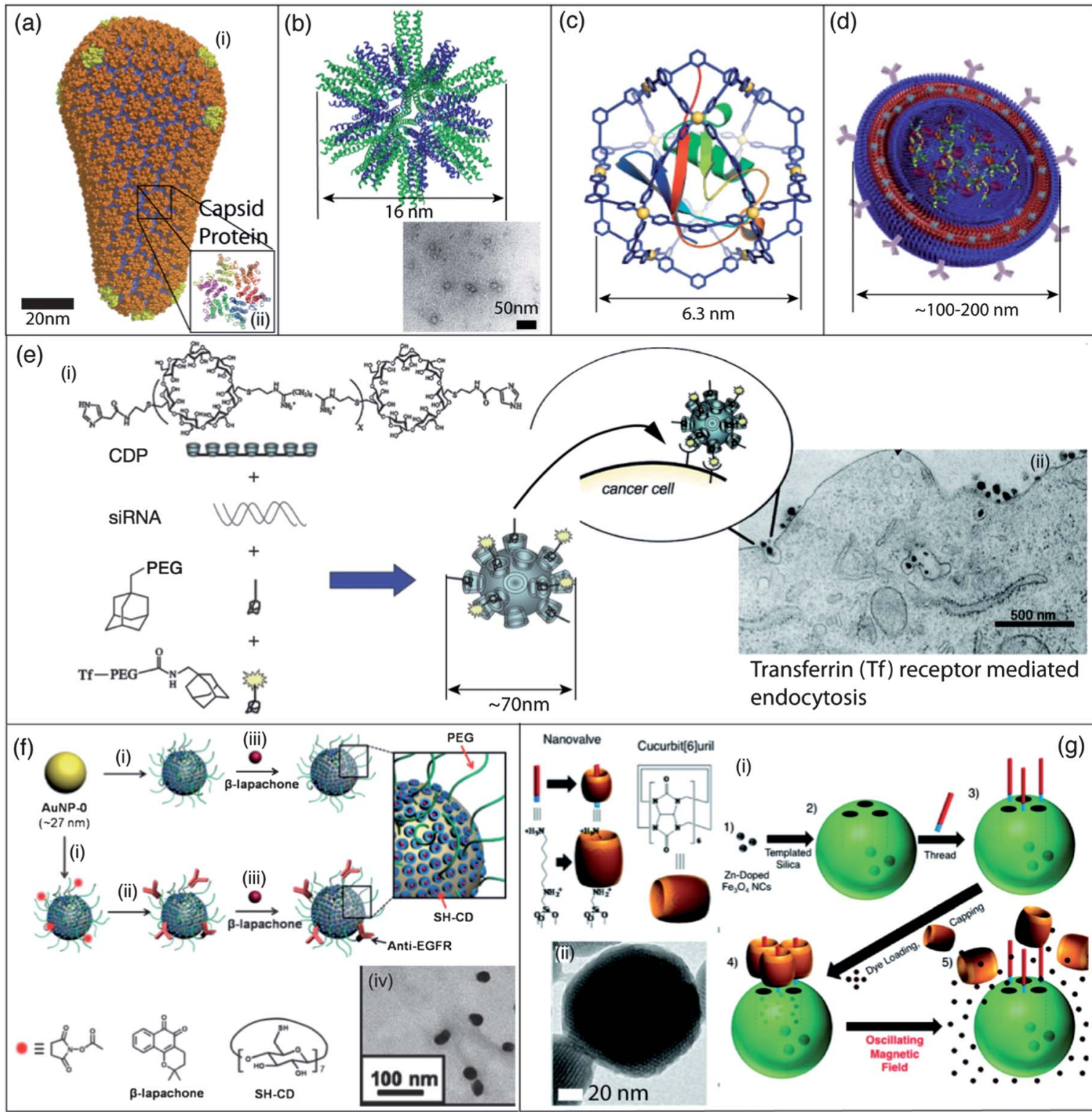

(g)

Fig. 2 Self-assembled nanomaterials for gene and drug delivery. (a) (i) Atomic level molecular modeling of a type-1 HIV viral particle, a member of the lentivirus family; ${ }^{87}$ (ii) crystal structure in the hexameric form of disulfide type-1 HIV CA protein, one of the building blocks in the viral capsid. Self-inactivating mutants of the type1 HIV virus are currently used for clinical gene therapies. (b) Molecular model of a peptide nanoparticle with dodecahedral symmetry formed by the self-assembly of a coiled-coil peptide with a pentameric domain (green) and a trimeric domain (blue) covalently linked to the same molecule and TEM micrograph of these peptide nanoparticles. (c) Molecular model of a discrete synthetic M12L24 nanocage containing a ubiquitin covalent conjugate. Self-assembly of Pd(I) ions (M) and additional ligands (L) results in the encapsulation of the protein into a well-defined coordination cage. (d) Schematic illustration of a polymersome decorated with antibodies for targeted delivery, and containing multiple cargos through incorporation into the hydrophobic portion of its membrane or through physical entrapment into its core. (e) (i) Schematic representation of the one-step self-assembly of four components into a targeted nanoparticle by the electrostatic interaction between siRNA and a cationic cyclodextrin containing polymer (CDP) and their inclusion complexes with adamantane (AD) derivatives on the surface of the nanoparticles; (ii) TEM micrograph of nanoparticles entering a cancer cell by receptor-mediated endocytosis. (f) (i) Functionalization of the surface of gold nanoparticles (AuNPs) with a cyclodextrin thiol derivative (SH-CD) and PEG-thiol (mPEG-SH or NHS-PEG-SH) chains; (ii) targeting was achieved through covalent decoration with anti-EGFR antibodies; (iii) a hydrophobic anti-cancer drug, $\beta$-lapachone can then be encapsulated into the hydrophobic cavity of the cyclodextrin; (iv) TEM micrograph of nanoparticles covered with cyclodextrin and PEG chains and loaded with $\beta$-lapachone. (g) (i) Preparation of mechanized silica nanoparticles loaded with a dye and zinc-doped iron oxide nanocrystals (ZnNCs). After attachment of the base of the molecular machine, the dye is loaded into the particle and the pores are blocked with the cucurbit(6)uril cap. Local heating upon the application of an oscillating magnetic field results in the dissociation of the nanovalve and release of the dye; (ii) TEM micrograph of ZnNCs encapsulated within mesoporous silica. Panel (a): reproduced with permission from ref. 87; panel (b): reproduced with permission from ref. 98; panel (c): reproduced with permission from ref. 101; panel (d): reproduced with permission from ref. 138; panel (e): reproduced with permission from ref. 136; panel (f): reproduced with permission from ref. 144; panel (g): reproduced with permission from ref. 148.

structures has been studied in detail. ${ }^{115,116}$ Among the different nucleic acids which can be used therapeutically, siRNA delivery is nowadays one of the most promising therapeutic approaches for a wide range of diseases and therefore the delivery of siRNA by cationic liposomes is under intense investigation, ${ }^{118}$ in particular to develop anti-cancer treatments. ${ }^{119,120}$ Because safe 


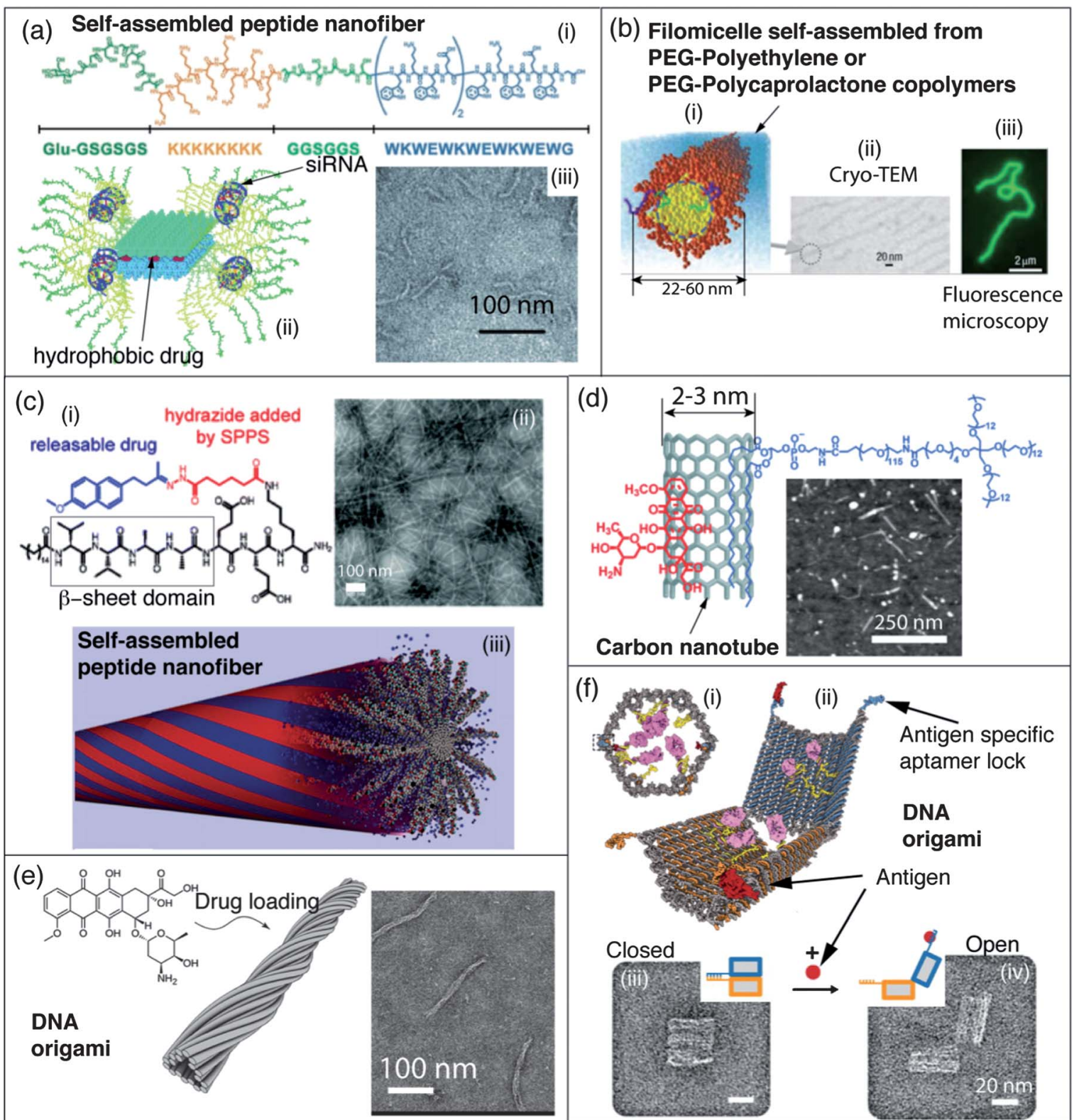

Fig. 3 Self-assembled nanomaterials for gene and drug delivery. (a) (i) Structure of self-assembling peptide Glu-KW with a $\beta$-sheet segment (blue), flexible linker (light green), siRNA binding domain (orange) and hydrophilic targeting domain (dark green); (ii) schematic representation of artificial filamentous nanostructures used for siRNA delivery; (iii) TEM micrograph of filamentous nanostructures obtained with peptide Glu-KW. (b) (i) Schematic representation of filomicelles obtained by selfassembly of block copolymers used to probe the effect of nanostructures' shape for drug delivery in vivo; (ii) cryo-TEM micrograph of such a filomicelle, (iii) these filomicelles can be labelled with a hydrophobic fluorescent dye and observed by fluorescence microscopy. (c) (i) Molecular structure of a peptide amphiphile conjugated to a drug through a reversible covalent bond; (ii) TEM micrograph of self-assembled peptide nanofibers; (iii) this peptide amphiphile forms self-assembled peptide nanofibers in water. (d) Molecular structure of an anticancer drug (doxorubicin (DOX)) bound through stacking and hydrophobic interactions to a carbon nanotube (SWNT) dispersed by a PEGylated amphiphile, and an AFM micrograph of SWNT-DOX complexes. (e) Molecular structure of the anticancer drug doxorubicin, and schematic representation of a filamentous DNA origami (T-Nano) able to store doxorubicin, presumably via intercalation between nucleobases, and deliver it into cancer cells. A TEM micrograph of DOX loaded T-Nano DNA origami is given. (f) (i) Front view of a closed DNA origami nanorobot for the target transport of molecular payloads (antibody Fab' fragments in magenta, and gold nanoparticles in yellow); (ii) schematic representation of the open form of the nanorobot in which the aptamer lock is stabilized in a dissociated state by its antigen key (red), TEM micrographs of the corresponding (iii) closed and (iv) open states of the nanorobot. Panel (a): reproduced with permission from ref. 102; panel (b): reproduced with permission from ref. 157; panel (c): reproduced with permission from ref. 159 and 182 ; panel (d): reproduced with permission from ref. 168; panel (e): reproduced with permission from ref. 175; panel (f): reproduced with permission from ref. 177.

and effective siRNA delivery agents are needed, the group of Langer described combinatorial approaches to identify highly active cationic lipids for lipofection by screening a library of more than 1200 lipids obtained by Michael addition of polyamines on lipid-acrylates or acrylamides. ${ }^{121}$ The lipoplexes with a diameter around $50 \mathrm{~nm}$ were screened both in vitro and in vivo in rat, mouse, and non-human primates to identify promising compounds for local and systemic delivery of siRNA 
and miRNA. Clinically relevant siRNA-mediated gene silencing was achieved in non-human primates by the delivery of specific siRNAs against apolipoprotein B (a protein associated with increased risk of coronary artery disease) using cationic PEGylated liposomes with a diameter around 77-83 nm. ${ }^{122}$ Gene silencing through the receptor specific delivery of siRNA against gp46 to hepatic stellate cells by targeted PEGylated cationic liposomes with a diameter around 120-150 $\mathrm{nm}$ has also been used as an antifibrotic therapy for liver cirrhosis in a rat model. $^{123}$

Cationic peptides are another particular type of cationic amphiphile that is used for transfection. Short cationic amphipathic peptides, ${ }^{\mathbf{1 2 4 , 1 2 5}}$ obtained by linking hydrophobic cell penetrating sequences ${ }^{\mathbf{1 2 6}}$ derived from the fusion sequence of the HIV gp41 together with a nuclear localization sequence (KKKRKV) for nucleic acid binding, were shown to be efficient DNA, siRNA, and protein delivery agents. ${ }^{\mathbf{1 2 4}}$ While the morphology of the complexes is not well defined, their size can be controlled by the nucleic acid/peptide ratio to obtain particles with an average diameter of $100 \mathrm{~nm} .{ }^{\mathbf{1 2 4}}$ These cationic peptides were also used for the intracellular delivery of proteins. $^{\mathbf{1 2 4}}$

Polyplexes. Alongside cationic lipids, cationic polymers have also been widely used to prepare nanoscale self-assembled complexes, namely polyplexes, with nucleic acids. ${ }^{127-129}$ Cationic polymers, such as polyethylene imine, are polyelectrolytes and have the advantage of interacting with polyanionic nucleic acids in a multivalent manner yielding stable complexes. The effective block copolymers commonly used thus contain a nucleic acid binding block such as polylysine, together with a neutral hydrophilic block such as PEG to prevent the aggregation of the polyplexes. ${ }^{\text {129-131 }}$

Polyplexes in the form of stable and defined $45 \mathrm{~nm}$ polymeric micelles were prepared by Kataoka et al. by spontaneous selfassembly between poly(ethylene glycol)-block-poly(L-lysine) and siRNAs. ${ }^{132}$ Targeting was achieved by the introduction of a cycloArg-Gly-Asp (cRGD) peptide on the PEG terminus. Increased gene silencing ability, improved cell uptake, and broader subcellular distribution for the complexed siRNA were demonstrated in vitro in a tumor model as well as in vivo in mice.

Recently several research groups paid particular attention to the morphology and the related mechanism of formation of polyplexes. Shinkai et al. have shown that helicoidal polysaccharides, such as $\beta$-1,3-glucans, form one dimensional hetero-triple helix complexes consisting of two chains and one polynucleotide chain with various types of nucleic acids. ${ }^{\mathbf{1 3 3}}$ These well-defined helicoidal complexes were used for the in vitro delivery of functional oligonucleotides to various cell types and were chemically modified with a cationic or hydrophobic group to further enhance cellular uptake. ${ }^{133}$ Kataoka et al. demonstrated the quantized folding of plasmid DNA upon complexation with poly lysine-PEG block copolymers yielding mixtures of rod-like complexes with quantized length and toroids. ${ }^{131}$ Rod-like polyplexes of individual plasmid DNA molecules were described by de Vries et al. ${ }^{\mathbf{1 3 4}}$ They used genetically engineered protein diblock copolymers composed of a DNA binding polylysine $\left(\mathrm{K}_{12}\right)$ segment and a 400 amino acid hydrophilic colloidal block for the stabilization of the complexes. Although these complexes did not show transfection properties, this type of study elaborates new rules for the design of homogeneous nanoscale carriers for nucleic acids.

A good control over the morphology of polyplexes was obtained by Luijten et al. ${ }^{135}$ They were able to go from spherical complexes to elongated rod-like complexes from the same composition of the complexes, simply by changing the polarity of the solvent medium used for the complexation. Interestingly, they have shown that elongated complexes are more efficient than their spherical counterparts for in vivo gene delivery in a mouse model. ${ }^{135}$

But the most advanced supramolecular system for gene delivery was developed by the group of Davis et al. who were the first to achieve targeted siRNA silencing in human clinical trials. ${ }^{136,137}$ Before giving the injection to the patient, the therapeutic nanoparticles can be readily obtained by the spontaneous self-assembly in one pot upon mixing a solution of the siRNA and a solution of the three other components of the system: a cyclodextrin-containing polymer (CDP), a polythethylene glycol-adamantane steric stabilization agent, and a human transferring-polyethylene glycol-adamantane conjugate as a targeting ligand (Fig. 2e). The authors optimized the complex formation between cationic polycyclodextrin polymers and siRNA for the formation of homogeneous nanoparticles with a diameter around 50-70 $\mathrm{nm}$ for optimal uptake by the tumors. ${ }^{136}$ The modularity and the ease of formulation of these complexes demonstrate the potential of self-assembled nanomaterials for the development of functional therapeutic agents.

Polymersomes and dendrimersomes. Polymersomes are vesicles formed by the self-assembly of amphiphilic block copolymers (Fig. 2d). ${ }^{\mathbf{1 3 8}}$ They share similar architectures and applications with their lipid counterparts, ${ }^{\mathbf{1 3 8}}$ but they are kinetically rather stable, as their dissociation would expose large hydrophobic macromolecular segments to the aqueous phase. As for liposomes, polymersomes can be loaded with hydrophilic or hydrophobic drugs, functionalized with PEG segments for prolonged circulation times, and decorated with targeting moieties on their surface. ${ }^{138}$ While a wide range of morphologies are accessible by the self-assembly of block copolymers, Percec et al. have also demonstrated the utility of dendritic structures to obtain impressive structural diversity by the self-assembly of easily accessible amphiphilic Janus dendrimers. ${ }^{139}$ They screened combinatorial libraries of Janus dendrimers by cryo-TEM and obtained dendrimersomes, cubosomes, disks, tubular vesicles and helical ribbons, making this system of high interest for drug delivery and other biomedical applications where shape plays a crucial role in the nanoparticle function.

Gold nanoparticles. Gold nanoparticles can serve as versatile scaffolds for the delivery of drugs and nucleic acids as they can bind molecular cargos via a wide range of non-covalent interactions. ${ }^{16,140-142}$ For example, gold nanoparticles with a diameter of $2.5 \mathrm{~nm}$ have been decorated with an amphiphilic monolayer composed of a hydrophobic alkanethiol linked to a hydrophilic tetra(ethylene glycol). ${ }^{\mathbf{1 4 3}}$ These constructs can transport hydrophobic drugs, such as tamoxifen, located in the dense 
hydrophobic corona at the gold surface, while the external PEG corona ensures the solubility of the drug loaded nanoparticles. The surface of gold nanoparticles ( $27 \mathrm{~nm}$ in diameter) can also be decorated by small receptors for hydrophobic drugs such as per-6-thio- $\beta$-cyclodextrin, by an antifouling PEG shell, and by targeting residues (Fig. 2f). ${ }^{\mathbf{1 4 4}}$ The cyclodextrin drug pocket can then be filled with an anticancer drug ( $\beta$-lapachone) and the release of the drug from the carrier surface is induced by goldthiol ligand exchange with the glutathione present in the intracellular matrix of cancer cells. ${ }^{\mathbf{1 4 4}}$ Gold nanoparticles are also extremely promising scaffolds for the delivery of functional nucleic acids immobilized at their surfaces by electrostatic interactions, hybridizations, or covalent thiol-gold bonds. ${ }^{\mathbf{1 4 5}}$ Mirkin et al. recently achieved a breakthrough in topical gene silencing using such spherical nucleic acid nanoparticle conjugates (SNA-NCs) prepared by the covalent immobilization of siRNA onto gold nanoparticles. ${ }^{\mathbf{1 4 6}}$ SNA-NCs penetrate keratinocytes with high efficiency with no associated cytotoxic effect. The application of these SNA-NCs, mixed with a commercial moisturizer, on skin leads to the knockdown of the epidermal growth factor receptor at very low concentrations in a mouse model, ${ }^{\mathbf{1 4 6}}$ demonstrating the enormous potential of SNA-NCs in treating skin diseases by siRNA mediated gene silencing.

Silica nanoparticles. Within the toolbox of supramolecular chemistry, the mechanical bond has been the latest one to be used for designing drug delivery platforms. Stoddart et al. described the use of mechanically interlocked molecules as stimuli responsive stoppers or nanovalves at the extremity of the pores in mesoporous silica nanoparticles. The resulting mechanized silica nanoparticles can respond to redox, light, base, or acid triggers in order to release drugs such as doxorubicin in vitro or in vivo. ${ }^{147} \mathrm{~A}$ particularly elegant approach for the stimuli responsive release of cargos was achieved by loading mechanized silica nanoparticles with magnetic zinc-doped iron oxide nanocrystals. This system enables the targeted release of doxorubicin in response to local heating upon exposure to an oscillating magnetic field (Fig. $2 \mathrm{~g}$ ). ${ }^{\mathbf{1 4 8}}$

Spherical micelles. Water soluble spherical micelles, obtained by the self-assembly of amphiphilic block copolymers, can serve as nanocontainers where hydrophobic drugs are loaded in their hydrophobic core. ${ }^{149}$ These self-assembled objects can be crosslinked by reversible or non-reversible covalent bonds to improve their stability. ${ }^{\mathbf{1 5 0}}$ The ability of micelles to release drugs by responding to stimuli such as temperature ${ }^{\mathbf{1 5 1}}$ has been extensively reviewed. ${ }^{\mathbf{1 5 2 , 1 5 3}}$ Recently, a new class of DNA diacyllipidmolecular-beacon conjugate amphiphile has been shown to form spherical micelles displaying DNA hairpins. ${ }^{154}$ Upon hybridization with a target mRNA, DNA hairpins undergo a conformation change which results in fluorescence emission. By scavenging intracellular mRNA, these sensing devices also modulate gene expression and are therefore applicable for gene therapy. ${ }^{154}$ In another example of polymeric DNA micelles, Gianneschi et al. showed that, as for the SNA-NCs described above, the dense array of nucleic acids displayed at the micellar surface confer interesting properties such as resistance to nucleases, ${ }^{155}$ suggesting their potential for gene delivery applications.
Cylindrical micelles. One dimensional nanostructures, ${ }^{\mathbf{1 5 6}}$ and in particular self-assembled cylindrical polymeric micelles, ${ }^{157}$ are effective carriers for drug delivery in vitro or in vivo. Discher et al. were the first to clearly show the advantage of using cylindrical micelles (or filomicelles), versus their spherical counterparts, to load and carry hydrophobic drugs to tumors in vivo (Fig. 3b). ${ }^{157}$ Other one dimensional platforms have been used for drug delivery and in particular, the Stupp group developed particularly interesting peptide nanofibers formed by the self-assembly of peptide amphiphiles (PAs). ${ }^{71}$ Upon charge screening, these PAs self-assemble into cylindrical micelles stabilized by extended $\beta$-sheet hydrogen bond networks. The PA nanofibers are degradable by cells and not immunogenic; they have been used for the controlled release of therapeutic carbon monoxide ${ }^{158}$ and the anti-inflammatory drug nabumetone ${ }^{159}$ in vitro by taking advantage of reversible covalent or coordination bonds between drugs and PAs (Fig. 3c). The anticancer drug camptothecin has also been loaded into the hydrophobic core of the PA cylindrical micelles by non-covalent interactions. ${ }^{160}$ Enzyme responsive PA nanofibers were designed by incorporating a consensus substrate sequence for protein kinase A, a potential cancer biomarker. Upon phosphorylation, electrostatic repulsion between peptide monomers triggers the disassembly of the nanofibers demonstrating their dynamic and stimuli responsive character. These fibers were shown to encapsulate a hydrophobic anticancer drug, doxorubicin, and release it upon protein kinase A treatment. This property was responsible for enhanced toxicity against cancer cells known to release large amounts of protein kinase A into their media. ${ }^{\mathbf{1 6 1}}$ The co-assembly of a cationic membrane lytic PA with a serum protective PEGylated PA helped in stabilizing the therapeutic cytotoxic peptide against protease degradation, therefore enhancing stability of the nanostructures in vitro. ${ }^{\mathbf{1 6 2}}$ These cytotoxic nanofibers were then shown to significantly reduce tumor cell proliferation in vivo in a mouse model. Dilution by co-assembly also allows reducing the proportion of cytotoxic PAs while retaining similar tumoricidal activity in vitro.

Recently, an original approach to increase the loading of drugs into self-assembled nanocarriers was discussed by Cui et al. ${ }^{163}$ They proposed to make the drug itself an integral part of the self-assembling core of the molecules. As a proof of principle, a hydrophobic drug, camptothecin, was used to replace the hydrophobic tail in the design of a PA to obtain a drugamphiphile (DA), allowing for higher drug content in the final self-assembled nanostructure. Cui et al. convincingly demonstrated this concept for the preparation of well-defined nanostructures from dendritic DA including nanofibers and nanotubes with different camptothecin contents. They also demonstrated the efficiency of these nanostructures against a number of cancer cell lines. ${ }^{\mathbf{1 6 3}}$

Carbon nanotubes. Carbon nanotubes have been used as onedimensional nanocarriers for gene ${ }^{\mathbf{1 6 4 , 1 6 5}}$ and drug delivery. ${ }^{\mathbf{1 6 6 - 1 6 8}}$ The use of carbon nanotubes requires dispersion in water by using covalent modification or supramolecular interaction with large amphiphiles, such as phospholipid PEG conjugates, to ensure sufficient solubility and stability. The extended aromatic 
surface of single wall carbon nanotubes (SWNTs) is also ideal for supramolecular $\pi-\pi$ stacking interactions with aromatic drugs such as doxorubicin (DOX). Higher loading capacities were obtained compared to other nanocarriers like liposomes or micelles, and DOX-loaded SWNTs display an enhanced therapeutic antitumoral effect and less toxicity than the free DOX (Fig. 3d) ${ }^{167,168}$ Dai et al. have also proposed a new concept of "functionalization partitioning"167 of SWNTs which consists in the co-assembly on the same nanotube of different lipid conjugates to confer multiple properties in the targeting delivery of drugs and genes. For example, SWNTs can be loaded via non-covalent interactions with radiolabels, antibodies, or siRNA conjugated with phospholipids. ${ }^{165}$ Covalent modification of carbon nanotubes with cationic chains allows the electrostatic binding of negatively charged nucleic acids. For example, Bianco et al. have demonstrated that complexes of plasmids and ammonium-functionalized carbon nanotubes enter cells by an endosome-independent mechanism, facilitate DNA uptake, and induce gene expression. ${ }^{164}$

Gels for drug delivery. Macroscopically stable gels are suitable for some delivery applications as their porous structures allow the controlled release of a therapeutic agent over extended periods of time. ${ }^{169,170}$ In particular, self-assembled gelators are of high interest because they can potentially be formed by in situ gelation of the building blocks. This could translate into clinical applications where an injection of soluble building blocks results in the gelation of the drug delivery materials by the formation of self-assembled nanostructures. In this context, gels formed by the self-assembly of peptides or polypeptides are very popular as they can be degraded and are biocompatible. ${ }^{171}$

Recent developments can be illustrated by the encapsulation of curcumin in self-assembling $\beta$-hairpin peptide hydrogels described by Pochan et al. ${ }^{172}$ The authors have demonstrated multiple modes of interactions between the hydrophobic curcumin and the valine rich $\beta$-hairpin MAX8. They were also able to control the release rate and toxicity against cancer cells of the bioactive guest by changing the concentration of the gelating peptide. Drug release from gels prepared by the network formation of self-assembled peptide nanofibers conjugated to drugs by reversible covalent bonds has also been described. ${ }^{159}$ In particular, physical gels have been prepared using charge screening by addition of $\mathrm{CaCl}_{2}$ and the release of nabumetone was observed over more than 27 days in vitro. ${ }^{159}$

For gene delivery, the localized and long-term delivery of siRNA against cyclin B1 by hydrogels was obtained by the gelation of thermoresponsive polyplexes. ${ }^{173}$ Complexes between siRNA and L-PEI-poly(organophosphazene) have shown a temperature-dependent sol-gel phase transition. The change in temperature upon injection into a tumor xenograft model in vivo induces the aggregation of $100 \mathrm{~nm}$ polyplexes via hydrophobic interactions. Polyplexes are then released from the hydrogel allowing the silencing of cyclin B1 for 4 weeks around the site of injection.

DNA-based nanomaterials. In recent years, nucleic acids selfassembly has emerged as the most powerful and predictable tool for the aqueous construction of complex nanostructures. This progress, together with the fact that nucleic acids are biocompatible, degradable, and in some cases bioactive as well, make them ideal building blocks for the development of nanoscale drug delivery vehicles. ${ }^{174}$ Because DNA self-assembly allows the preparation of self-assembled nanostructures with a perfect control over the size and shape of the constructs, it represents a crucial advantage when these parameters can affect the bioactivity of the nanostructures. For example, monodisperse nanofilaments were prepared by Högberg et al. using the principles of DNA origami and loaded with doxorubicin by intercalation between the nucleobases within the DNA nanostructure (Fig. 3e). ${ }^{175}$ These one dimensional nanostructures can penetrate breast cancer cells to promote apoptosis.

To design an ideal nanoscale drug carrier, one would imagine a tridimensional structure that could be filled with a therapeutic agent or solution, closed, and then selectively reopened at the targeted delivery site. The first prototype of such a DNA nanobox, that could potentially be used as a delivery vector, was described by Gothelf and Kjems who designed a DNA origami nanocube with a controllable lid. ${ }^{176}$ This lid could be held in a closed position by a set of two locks composed of DNA duplexes with stickyend extensions, allowing the opening of the lid by the dissociation of the duplex upon externally added "key" oligonucleotides. More recently, DNA aptamers have been used as logic gates to control the opening and closing of self-assembled targeted nanorobots (Fig. $3 \mathrm{f}){ }^{177}$ Here the keys that trigger the opening of a self-assembled DNA barrel are two orthogonal aptamers. Therefore, both aptamers have to bind to their target (a protein for example) in order to induce the opening of the DNA barrel and the exposure of the biologically active payload, such as antibodies. This construct, loaded with a combination of an antibody to human CD33 and an antibody to human CDw328 Fab' fragments, is able to induce growth arrest in NKL large granular lymphocytic leukemia cells in a dose-dependent fashion. ${ }^{177}$

2.2.2 Regenerative medicine and tissue engineering. Nanomaterials have been extensively used in tissue engineering. ${ }^{178,179}$ Due to their dimensions, they have unique abilities to mimic some of the extracellular matrix components, and to direct cell adhesion and morphology. ${ }^{180}$ In particular, selfassembled nanomaterials offer some additional advantages because of their kinetic lability, giving access to properties such as self-construction, degradability, and adaptivity. ${ }^{181,182}$ Selfconstruction implies that their assembly can be achieved in situ in the presence of cells or in vivo after injections of their molecular components at a given site. Their reversible nature facilitates their dissociation into their molecular constituents, allowing traceless degradation once they are no longer of use in vivo. Another property of dynamic systems is their ability to adapt to their environment; for example, a cell surface covered with receptors can potentially induce the clustering of its ligands on the surface of a self-assembled structure to maximize binding between the two surfaces.

Nanofibers. Among all examples of self-assembled nanomaterials for tissue engineering and regenerative medicine, self-assembled peptide nanofibers are the most common. ${ }^{71,72,171,183-186}$ They are built by the self-assembly of relatively simple peptidic building blocks, readily obtained by fast and reliable synthesis, which in turn facilitates the 
introduction of peptidic or non-peptidic epitope for specific applications. In addition, their peptidic constitution confers them unique biocompatibility and degradability as they can in principle be used as nutrients by cells. The design of selfassembling peptides usually involves the use of short peptide sequences known to facilitate the formation of secondary structures like $\beta$-sheets or $\alpha$-helices, and their conjugation to hydrophobic chains in order to promote their aggregation in water. The peptide amphiphiles (PAs) developed by the Stupp group are based on a self-assembling platform composed of an aliphatic hydrophobic chain conjugated to a peptide sequence with $\beta$-sheet propensity and rich in hydrophobic acids, such as valine, leucine and alanine. ${ }^{71}$ PAs self-assemble into high aspect ratio nanofibers that can mechanically support cells and interact with cell receptors through the display of peptidic or non-peptidic biological signals that are easily introduced at the other extremity of the molecule. PAs also form gels upon charge screening which has led to the preparation of self-assembled biomaterials for spinal cord,$^{187}$ bone, ${ }^{188}$ cartilage, ${ }^{189}$ blood vessels, ${ }^{190}$ heart, ${ }^{191}$ islets ${ }^{192}$ and enamel regeneration. ${ }^{193}$

More recently, a VEGF-mimetic PA has been used to prepare nanofibers that promote proangiogenic behavior in endothelial cells in vitro, and an angiogenic response in vivo both in a chicken embryo assay and in an ischemic hind limb mouse model (Fig. 4a). ${ }^{194}$ PA nanofibers can also form highly anisotropic materials, a feature potentially useful when designing biomaterials to regenerate tissues with architectures based on cell alignment such as spinal cord, blood vessels, and muscles. For example, shear forces applied to PA nanofiber liquid crystals obtained by heating and cooling a PA solution induced the formation of highly aligned arrays of nanofibers that can be mixed with cells and stabilized by gelation with $\mathrm{CaCl}_{2}$, resulting in macroscopic monodomain gel strings, in which the nanofibers and cells are aligned (Fig. 4b). ${ }^{\mathbf{1 9 5}}$ PA nanofibers based liquid crystals can also be organized radially in tubular constructs and used to align smooth muscle cells (SMCs), mimicking the circumferential alignment of contractile SMCs necessary for the design of artificial blood vessels. ${ }^{\mathbf{1 9 6}}$

Another important problem in tissue engineering and regenerative medicine is to protect grafts and cells upon transplantation. Therefore, it is of interest to design biomaterials that could encapsulate cells, protect them from the host immune system, while allowing nutrients to diffuse in and out of the capsule. Such architectures have been prepared by the Stupp group in the form of macroscopic sacs that can then be used to encapsulate cells. ${ }^{197}$ Sacs were obtained by the selfassembly of a negatively charged megadalton biopolymer and positively charged peptide amphiphiles. Upon mixing individual solutions of these two components, the formation of a diffusion layer prevents random mixing of the two solutions, and results in the formation of highly ordered membranes decorated with bundles of peptide nanofibers oriented at a $90^{\circ}$ angle to the membrane. Such highly ordered hierarchical membranes obtained by the diffusion controlled self-assembly between a mixture of hyaluronic acid, heparin, and a heparinbinding PA, have been used recently to promote angiogenesis in the chicken chorioallantic membrane assay. ${ }^{198}$ Another class of self-assembling peptide has been introduced by Zhang et al. ${ }^{199}$ The self-assembling core of this class of peptide amphiphile is composed of repetitive sequences such as -AEAEAKAKAEAEAKAK- or -RARADADARARADADADA- that are self-complementary due to the presence of both positive and negative side chains on one side of the $\beta$-sheet, and hydrophobic side chains on the other. This self-assembly platform can be decorated with bioactive epitopes and used as 3D cell culture media for a wide range of cell types for tissue engineering. ${ }^{186}$ The self-assembling peptide scaffolds RADA16 and PRG peptide significantly enhance mouse pre-osteoblast cell proliferation and differentiation as well as stimulate cell migration into the 3D scaffold (Fig. 4c). ${ }^{200}$ More recently, Hartgerink et al. introduced a new method to recreate collagen fibers and gels from the selfassembly of simple peptides with sequences derived from the collagen's proline-hydroxyproline-glycine repeating unit. These peptides self-assemble into triple helices having electrostatic sticky ends that promote the elongation of triple helices involving salt-bridged hydrogen bonds between lysine and aspartate (Fig. 4d). ${ }^{201}$ Owing to the major role of collagen matrices in tissue engineering, this strategy is promising for the preparation of novel collagen analogs in regenerative medicine.

\section{Self-assembled nanomaterials for information technologies}

Information technologies based on optics and electronics play a key role in our modern society. In particular, organic (opto)electronics is a promising field of research with a high potential for the fabrication of new devices with a low cost of production. To implement this field, the bottom-up construction of electroactive nanomaterials is becoming a very popular domain of investigation thanks to its potential for miniaturizing and improving the material characteristics. In this chapter we will describe a selection of the recent literature on self-assembled nanomaterials for information technologies along two main sections. In the first one, we will describe self-assembled nanomaterials for organic electronic devices ${ }^{\mathbf{2 0 2 - 2 0 4}}$ including conducting materials, organic field effect transistors, organic light emitting diodes, and organic solar cells. In the second section, we will focus on nanomaterials created for the storage and processing of information, ${ }^{\mathbf{2 0 5}, 206}$ including memory devices and Boolean logic circuits.

\subsection{Organic electronics}

Conducting and semiconducting organic materials have recently attracted a growing number of research investigations because of their potential for the production of low cost and flexible electronic devices. Among these materials, self-assembled architectures can improve electronic properties by affording long-range ordering of molecules and by providing additional tuning parameters in order to control the devices' properties. In the following sections, we discuss some recent examples of supramolecular electroactive nanostructures displaying various electronic properties as conductive materials, field-effect transistors, light-emitting diodes, and photovoltaic devices. 


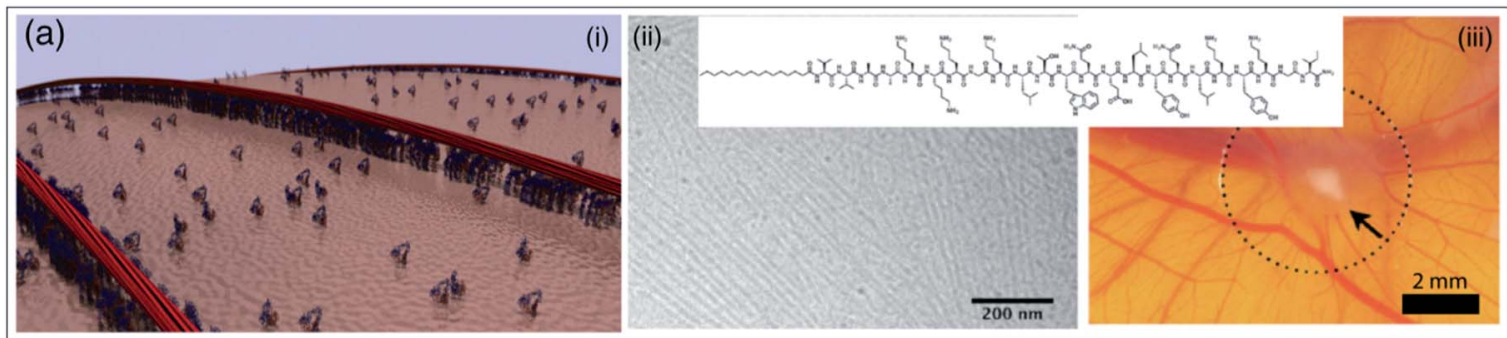

(b)

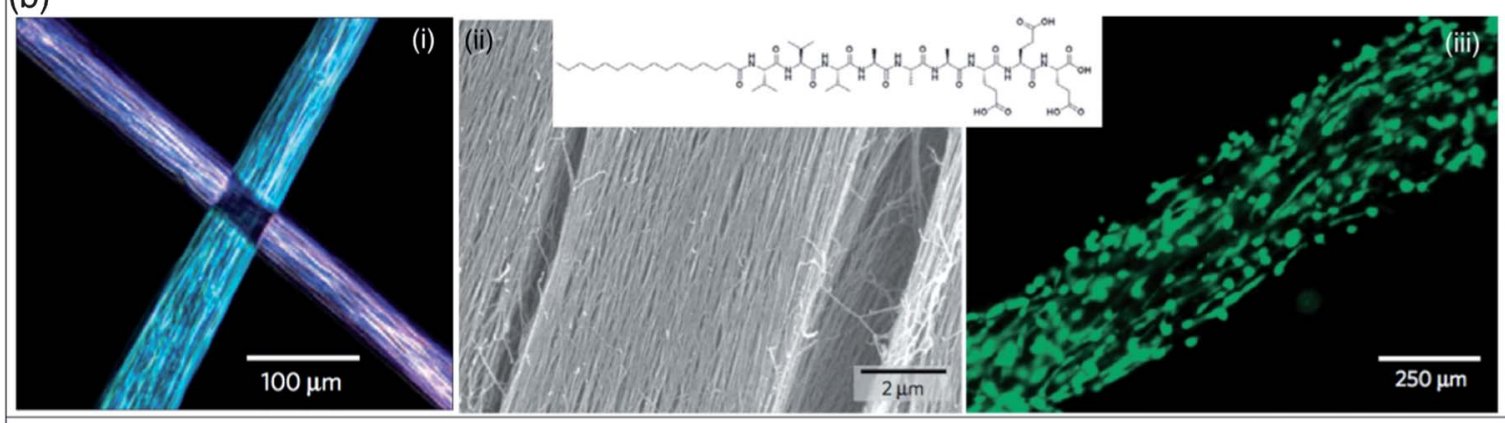

(c) (i) RADA 16

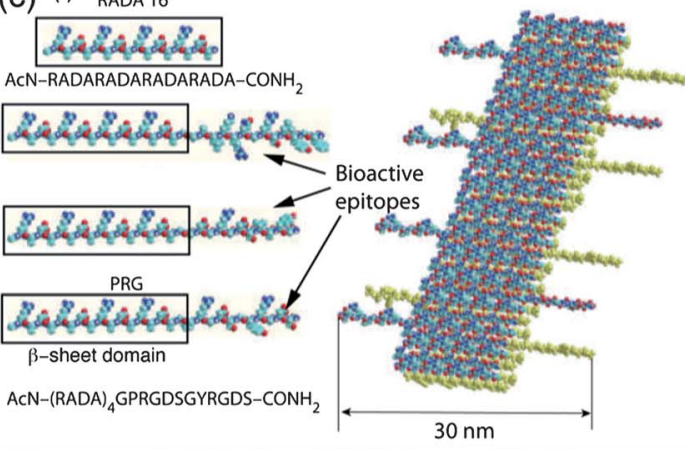

(ii)
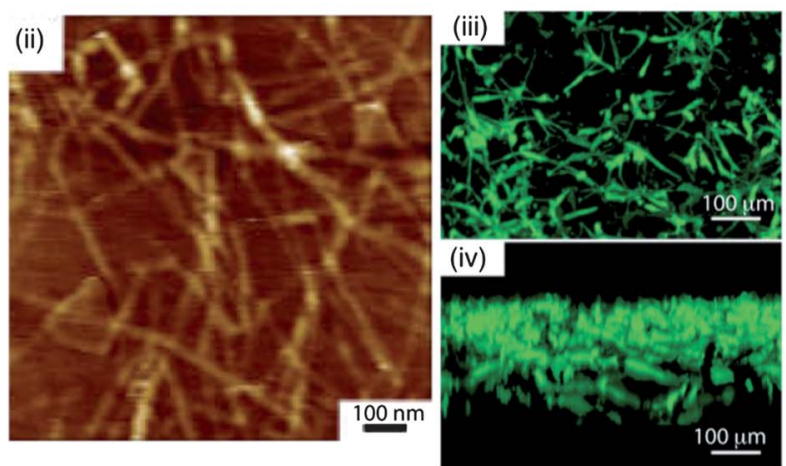

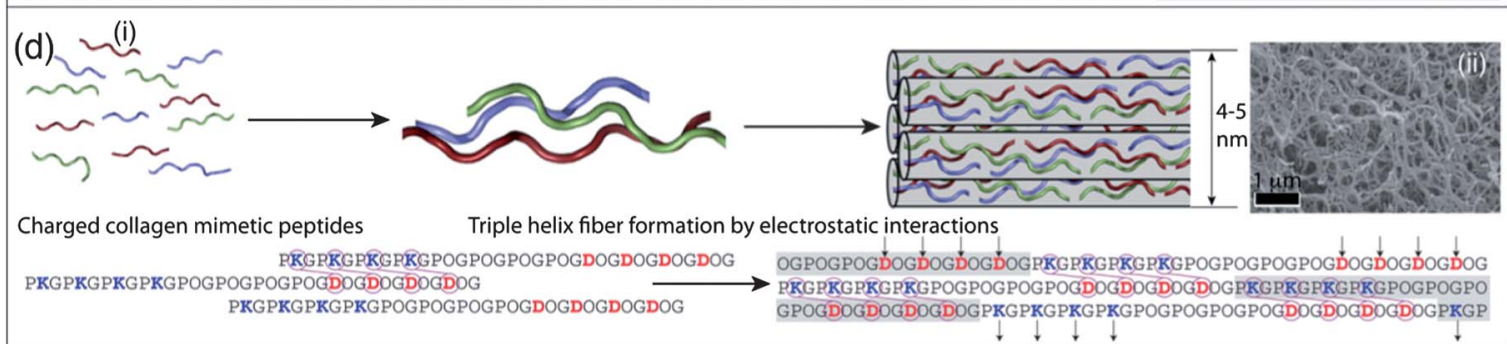

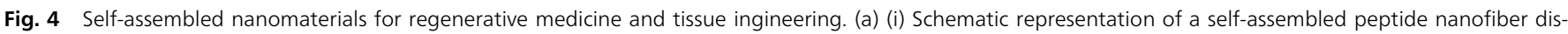

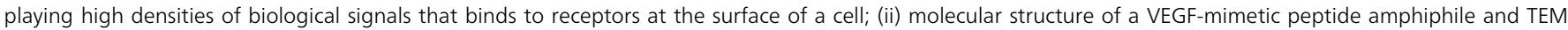

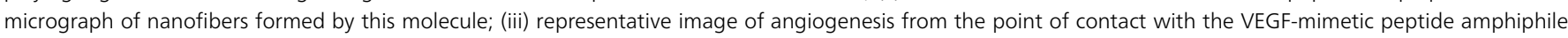

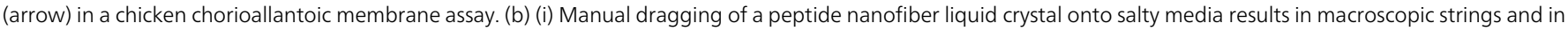

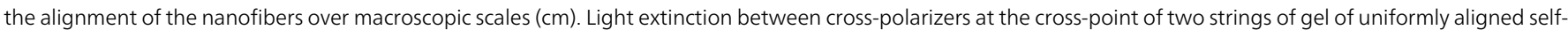

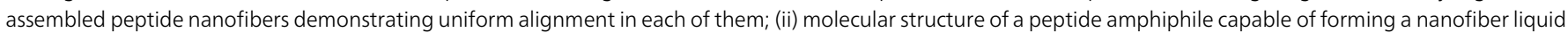

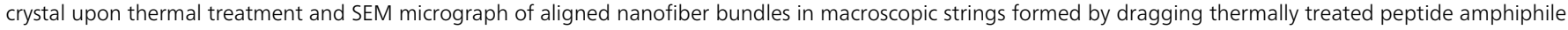

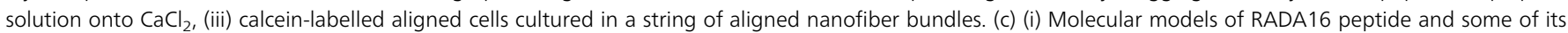

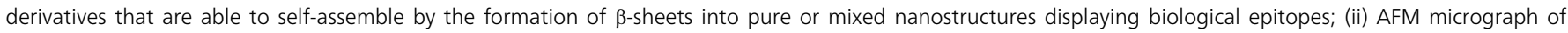

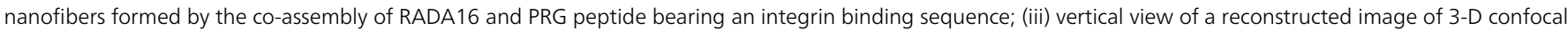

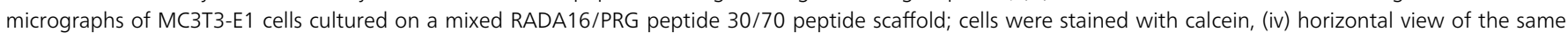

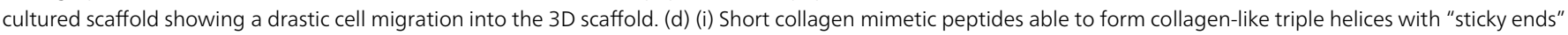

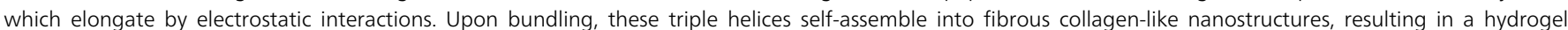

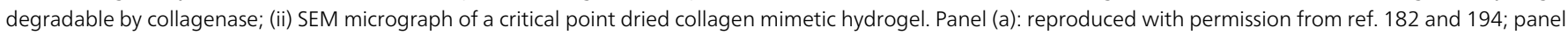
(b): reproduced with permission from ref. 195; panel (c): reproduced with permission from ref. 200; panel (d): reproduced with permission from ref. 201.

3.1.1 Supramolecular conducting materials. Much effort has been made toward the development of conducting organic materials during the past decades. ${ }^{207}$ In addition to films of conducting polymers (plastic electronic), and single molecules (molecular electronic), the emergence of self-assembled conductive materials (supramolecular electronics) represents a 
promising field of investigation which was reviewed recently. ${ }^{208-211}$

Nanofibers. Giuseppone and co-workers have reported the synthesis of an entirely new class of nanostructures based on triarylamine stacks, as well as their addressable selfconstruction between two electrodes, which represents a major progress to control supramolecular electroactive materials at the $100 \mathrm{~nm}$ length scale (Fig. 5a)..$^{212-214}$ These supramolecular triarylamine nanowires (STANWs) are generated by a simple light irradiation allowing the production of a catalytic amount of triarylammonium radicals which then piles with their neutral counterparts along nucleation-growth and self-replication processes. Using these properties, the localization and directionality of the fibers' elongation can be spatially addressed by applying an electric field directly between electrodes. An exceptional channel conductivity exceeding $5 \times 10^{3} \mathrm{~S} \mathrm{~m}^{-1}$, and an interface resistance per unit of length below $2 \times 10^{-4} \Omega \mathrm{m}$ were measured for these self-constructing devices. ${ }^{215}$

Another important class of stacked molecules extensively studied as conductive materials are tetrathiafulvalene (TTF) derivatives. Iyoda $e t$ al. have synthesized a TTF hexamer which, after oxidation to its monocationic form in chloroform, readily gives a deep green fibrous material when self-assembled upon addition of hexane. ${ }^{216}$ These nanofibers of several micrometers in length and having a diameter of $50 \mathrm{~nm}$ revealed a hexagonally ordered lamellar structure with a stacking distance of $3.51 \AA$ and a conductivity of $0.11 \mathrm{~S} \mathrm{~m}^{-1}$. The conduction of the selfassembled structure is two orders of magnitude higher compared to thin-films made of the same molecule. Amabilino and co-workers studied a self-assembled organogelator composed of TTF-based nanofibers. ${ }^{217}$ Subsequent doping of these fibers with iodine leads to room-temperature conductivity of $0.5 \mathrm{~S} \mathrm{~m}^{-1}$ which could be improved by one order of magnitude after annealing. Finally, Park et al. reported a new TTFdiamine producing, after doping with perchlorate ions, mixed valence double and triple helices due to the spiral configuration of the doped nanofibers (Fig. 5b). ${ }^{218} \mathrm{~A}$ room temperature conductivity of $4.59 \times 10^{-3} \mathrm{~S} \mathrm{~m}^{-1}$ was obtained with these nanostrutures.

Nanotubes. Würthner and co-workers studied bacteriochlorophyll c (BChl c) derivatives equipped with hydrophilic wedges and which self-assemble in water to produce well-defined nanotubes having a diameter of $6 \mathrm{~nm}$ (Fig. $5 \mathrm{c}$ ). ${ }^{219}$ These nanostructures have shown conductivities up to $0.48 \mathrm{~S} \mathrm{~m}^{-1}$ and charge-carrier mobilities of $0.03 \mathrm{~cm}^{2} \mathrm{~V}^{-1} \mathrm{~s}^{-1}$. Aida et al. synthesised self-assembled nanotubes from covalent hexabenzocoronene ( $\mathrm{HBC})-\mathrm{C}_{60}$ dyads by using a heating-cooling process. ${ }^{220}$ This structure exhibits a photovoltaic response upon light irradiation and an ambipolar character has been determined with a hole mobility of $9.7 \times 10^{-7} \mathrm{~cm}^{2} \mathrm{~V}^{-1} \mathrm{~s}^{-1}$ and an electron mobility of $1.1 \times 10^{-5} \mathrm{~cm}^{2} \mathrm{~V}^{-1} \mathrm{~s}^{-1}$. Co-assembly of the previous $\mathrm{HBC}-\mathrm{C}_{60}$ dyad with another $\mathrm{HBC}$ derivative without fullerene also yields nanotubes. In this case, a flash-photolysis time-resolved microwave conductivity (FP-TRMC) technique ${ }^{221,222}$ was used to determine a hole mobility of $2 \mathrm{~cm}^{2} \mathrm{~V}^{-1} \mathrm{~s}^{-1}$ that is as large as the inter-sheet mobility in graphite.

Nanoribbons/nanorods. Yagai and co-workers have reported the interconvertion of oligothiophene nanorods and nanoribbons by using a supramolecular dynamic exchange. ${ }^{223}$ Highly intrinsic hole mobilities were measured by using the pulse radiolysis time-resolved microwave conductivity (PR-TRMC) technique ${ }^{224,225}$ with values of $1.3 \mathrm{~cm}^{2} \mathrm{~V}^{-1} \mathrm{~s}^{-1}$ for the nanorods

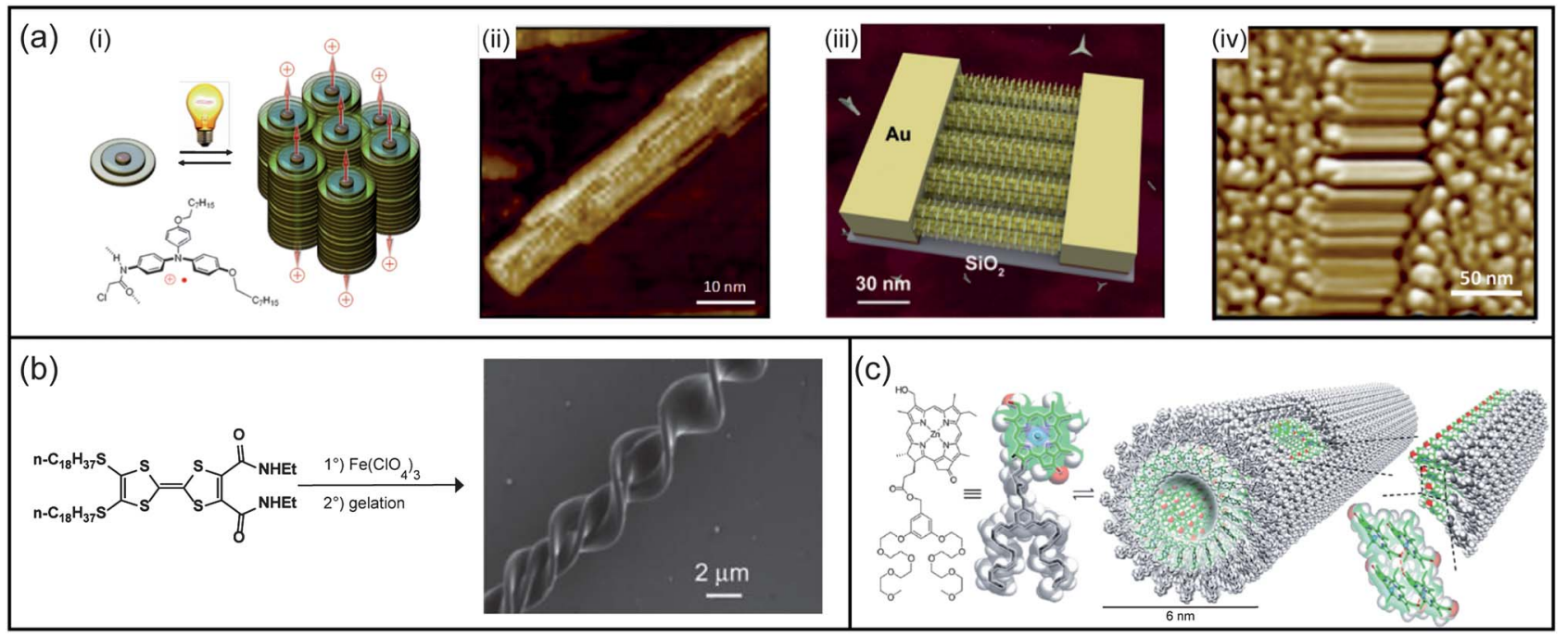

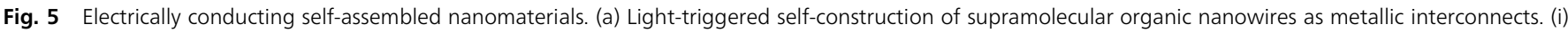

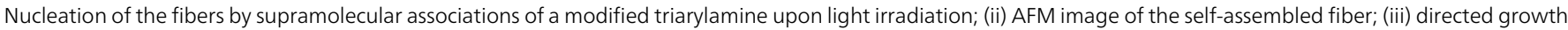

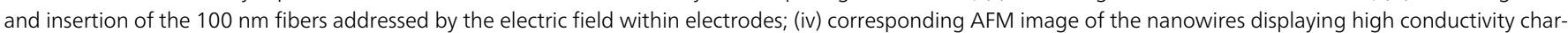

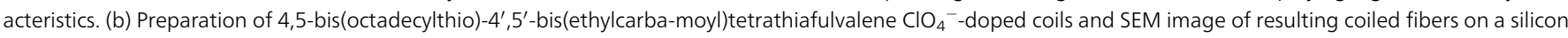

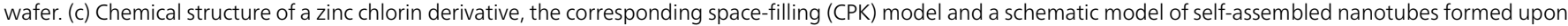

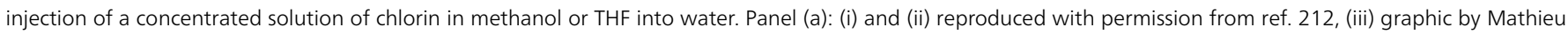
Lejeune. (iv) Reproduced with permission from ref. 213; panel (b): reproduced with permission from ref. 218 ; panel (c): reproduced with permission from ref. 219. 
and $0.57 \mathrm{~cm}^{2} \mathrm{~V}^{-1} \mathrm{~s}^{-1}$ for the nanoribbons. Zang et al. have studied other nanoribbons made from the self-assembly of perylene tetracarboxydiimide derivatives ${ }^{226}$ and which display a conductivity value of $1 \times 10^{-3} \mathrm{~S} \mathrm{~m}^{-1}$, whereas a supplementary treatment with saturated hydrazine vapours leads to an enhanced conductivity up to $0.5-1 \mathrm{~S} \mathrm{~m}^{-1}$.

3.1.2 Supramolecular organic field-effect transistors. The field-effect transistor is one of the most basic building blocks in electronics and nanoelectronics. In particular, organic fieldeffect transistors (OFETs) (227-229 are three-terminal components in which the current flow, through the active organic layer and between two of the terminals - known as the source and drain, is controlled by the bias applied to the third terminal - known as the gate. In addition, OFETs have an insulating layer between the semiconductor layer and the gate. The expected intrinsic properties of OFETs for practical use include high chargecarrier mobilities, large on-off current ratios, and threshold voltages close to zero, while maintaining good processability and low cost of the organic material. Many progresses have been made and several reviews were recently published regarding the implementation of functional supramolecular nanostructures in this type of device. ${ }^{208,210,230-233}$

Nanofibers. Fabrication of film-type and single-nanofiber OFETs from organogels based on anthracene derivatives has been described by Lee et al. (Fig. 6a). ${ }^{234}$ The supramolecular organization in fibers leads to higher performance (field effect mobility $0.48 \mathrm{~cm}^{2} \mathrm{~V}^{-1} \mathrm{~s}^{-1}$; on-off ratio $10^{5}$ ) compared to the film-casting versions. Samori and co-workers have shown that both processing techniques and side-chains have strong influences on the morphology of perylene tetracarboxydiimide (PDI) nanofibers and consequently, on the characteristic parameters of field-effect transistors. ${ }^{235}$ With another perylene tetracarboxylicdiimide derivative, Bao and co-workers have described the fabrication of single-crystalline wire OFETs. ${ }^{236}$ In this study, the average diameters and lengths of nanowires are respectively $519 \mathrm{~nm}$ and $340 \mu \mathrm{m}$, producing active layers with a mobility as high as $1.4 \mathrm{~cm}^{2} \mathrm{~V}^{-1} \mathrm{~s}^{-1}$. The same authors have also made use of a filtration-and-transfer method to align wires, leading to a maximum mobility of $0.24 \mathrm{~cm}^{-2} \mathrm{~V}^{-1} \mathrm{~s}^{-1}$ for multiple wire OFETs. Gong and co-workers have studied the self-assembly of a poly( $p$-phenylene ethynylene) derivative in crystalline nanowires with diameters comprised in the range of 5-15 $\mathrm{nm}$ and lengths up to hundreds of micrometers (Fig. 6b). ${ }^{237}$ Mobilities of these objects in OFET devices have shown values up to $0.1 \mathrm{~cm}^{2} \mathrm{~V}^{-1} \mathrm{~s}^{-1}$ which are several orders of magnitude higher than thin-film devices of the same polymer. Another example comes from Schenning et al. who have described the self-assembly of hydrogen-bonded oligo( $p$-phenylenevinylene) and perylene tetracarboxydiimide leading to thin-films with an internal rodlike morphology. ${ }^{238}$ These OFET devices have shown ambipolar transport, however with both low electron and hole mobilities $\left(10^{-7} \mathrm{~cm}^{2} \mathrm{~V}^{-1} \mathrm{~s}^{-1}\right)$.

Nanoribbons. Xia et al. have described the use of selfassembled nanoribbons from perylene tetracarboxydiimide in n-channel OFETs. ${ }^{239}$ In conjunction with p-channel hexathiapentacene nanowire OFETs, the authors have succeeded in producing a functional complementary inverter. Xia and co- workers have also reported long-term stable OFET devices based on nanobelt architectures prepared from poly(benzobisimidazobenzophenanthroline) polymers and with widths up to $1 \mu \mathrm{m}$ and lengths up to $150 \mu \mathrm{m}$ (Fig. 6c). ${ }^{240}$ Electron mobilities were measured for both single and networks of nanobelts with respective values of $7 \times 10^{-3} \mathrm{~cm}^{2} \mathrm{~V}^{-1} \mathrm{~s}^{-1}$ and $4 \times$ $10^{-3} \mathrm{~cm}^{2} \mathrm{~V}^{-1} \mathrm{~s}^{-1}$. Self-assembled oligoarene nanoribbons in single-wire OFETs were studied by Pei et al. ${ }^{\mathbf{2 4 1}}$ Mobilities up to $0.01 \mathrm{~cm}^{2} \mathrm{~V}^{-1} \mathrm{~s}^{-1}$ were obtained with an on-off ratio of $10^{3}$. An improved version of these OFETs was recently reported with a mobility as high as $2.1 \mathrm{~cm}^{2} \mathrm{~V}^{-1} \mathrm{~S}^{-1}$, an on-off ratio of $2 \times 10^{5}$, and a threshold of $-7 \mathrm{~V} \cdot{ }^{242}$ Self-assembled nanoribbons made of dithioperylene in OFET devices were reported by Pei et al. ${ }^{\mathbf{2 4 3}}$ Mobility as high as $2.13 \mathrm{~cm}^{2} \mathrm{~V}^{-1} \mathrm{~s}^{-1}$ and an on-off ratio of $10^{6}$ were obtained from a single-crystalline nanoribbon device.

Liquid crystals. Lee and co-workers reported a novel donoracceptor type semiconducting copolymer poly(didodecylquaterthiophene) forming highly crystalline thin films after thermal annealing. ${ }^{244}$ High field-effect mobilities of $0.33 \mathrm{~cm}^{2} \mathrm{~V}^{-1} \mathrm{~s}^{-1}$ were obtained as well as a good environmental stability and a bias-stress stability comparable to that of amorphous silicon. Discotic quaterrylene tetracarboxyldiimide was studied by Müllen et al. as an active layer leading to ambipolar OFETs. ${ }^{245}$ Electron and hole mobilities of respectively $1.5 \times$ $10^{-3} \mathrm{~cm}^{2} \mathrm{~V}^{-1} \mathrm{~s}^{-1}$ and $1 \times 10^{-3} \mathrm{~cm}^{2} \mathrm{~V}^{-1} \mathrm{~s}^{-1}$ were obtained. After annealing, the authors showed that only the electron transport behaviour remains with a mobility of $2 \times 10^{-4} \mathrm{~cm}^{2} \mathrm{~V}^{-1} \mathrm{~s}^{-1}$. Geng et al. have synthesised a discotic liquid crystalline phthalocyanato derivative which was used to make OFETs with mobility up to $0.017 \mathrm{~cm}^{2} \mathrm{~V}^{-1} \mathrm{~s}^{-1}$ after annealing. ${ }^{246}$ Imahori and co-workers have prepared donor-acceptor dyads from zinc phthalocyanine which self-assemble in columnar liquid crystals (Fig. 6d). ${ }^{247}$ The time-of-flight method (TOF) ${ }^{248}$ was used to determine efficient ambipolar charge-transport properties with an electron mobility of $0.26 \mathrm{~cm}^{2} \mathrm{~V}^{-1} \mathrm{~s}^{-1}$ and a hole mobility of $0.11 \mathrm{~cm}^{2} \mathrm{~V}^{-1} \mathrm{~s}^{-1}$.

Coordination complexes. Ma et al. have reported the use of two supramolecular triple decker complexes composed of tris(phthalocyanato) europium with crown ether heads and hydrophobic tails; both of them show good carrier mobilities (0.28$\left.0.33 \mathrm{~cm}^{2} \mathrm{~V}^{-1} \mathrm{~s}^{-1}\right) .{ }^{249}$ However, a noticeable difference between them concerns the direction of the current: in one case, it flows parallel to the aromatic phthalocyanine due to a J-aggregate arrangement whereas, in the second case, it flows parallel to the long axis of the complexes due to a $\mathrm{H}$-aggregate arrangement.

3.1.3 Supramolecular organic light-emitting devices. Organic light-emitting devices are promising candidates for next-generation display technology and solid-state lighting applications. ${ }^{250-254}$ These optoelectronic devices are currently divided between two classes. The first one is composed of organic light-emitting diodes (OLEDs) which are constituted of several semiconducting organic layers sandwiched between two electrodes. The second one makes use of light-emitting electrochemical cells (LECs) which consist of a single-layer of ionic luminescent material. Recently, self-assembled supramolecular materials for the construction of OLEDs and LECs have attracted a lot of attention and have been extensively reviewed. ${ }^{230,233,255-259}$ 


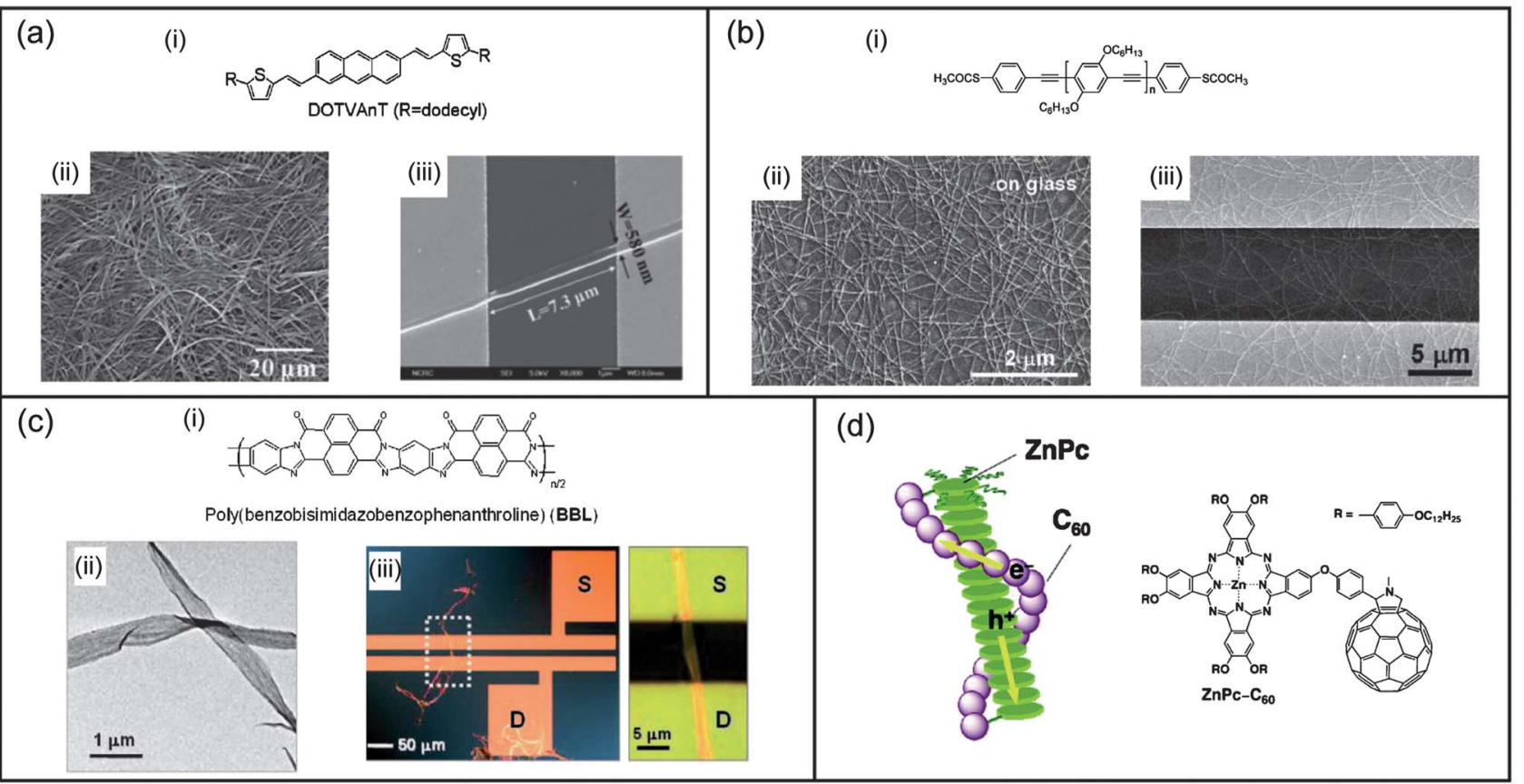

Fig. 6 Self-assembled nanomaterials for field-effect transistors. (a) (i) Structure of a 2,6-bis(2-thienylvinyl)anthracene derivative and SEM images of (ii) its xerogel and (iii) the bottom-contact single-nanofiber transistor of the considered molecule. (b) (i) Structure of the poly(para-phenylene ethynylene) polymer with thioacetate end groups; (ii) SEM images of the self-assembled nanowires on glass substrates from THF solution and (iii) the transistor device with nanowires between the source and drain electrodes. (c) (i) Structure of poly(benzobisimidazobenzophenanthroline); (ii) TEM image of the self-assembled nanoribbons; (iii) typical single nanoribbon transistor and a close-up showing the nanoribbon bridging the source-drain electrodes. (d) Structure and proposed schematic model of the liquid crystal arrangement of the considered phthalocyanine-fullerene dyad. Panel (a) reproduced with permission from ref. 234; (b) reproduced with permission from ref. 237; (c) reproduced with permission from ref. 240; (d) reproduced with permission from ref. 247

Nanofibers. Del Guerzo et al. have described the self-assembly of blends of tetracene derivatives leading to color-tunable organogels constituted of fluorescent nanofibers (Fig. 7a). ${ }^{260}$ Under near UV light excitation, both organogels and dry films of nanofibers emit white light, which is polarized in the blue spectral region. Additional confocal fluorescence microspectroscopy experiments showed that white light is emitted by each individual nanofiber. De Cola and co-workers have reported the use of a platinium(II) complex which self-assembles in bright gelating nanofibers as a dopant for solution-processed OLEDs. ${ }^{261}$ The highest efficiency with this device reached $15.6 \mathrm{~cd} \mathrm{~A}^{-1}$ at a brightness of $203 \mathrm{~cd} \mathrm{~m}^{-2}$. The self-assembly of a rigid hexaacid compound into supramolecular polymeric nanowires with diameters between 100 and $200 \mathrm{~nm}$ and lengths up to hundreds of micrometers was studied by Pei et al. (Fig. 7b). ${ }^{262}$ They showed a solid-state absolute photoluminescence (PL) efficiency of $22 \%$ representing only a loss of $10 \%$ against their solvent efficiency.

Supramolecular polymers. Schubert and co-workers have reported the study of $\pi$-conjugated bis(terpyridine) macroligands with poly( $\varepsilon$-caprolactone) on their side chains. ${ }^{263}$ The formation of supramolecular $\mathrm{Zn}$ (II) metallo-polymers with these macroligands leads to blue photoluminescence with high quantum yields both in diluted solution and solid state, demonstrating the suppression of the aggregation that usually occurs with metallo-polymers. Meijer et al. have also described a supramolecular copolymer resulting from the self-assembly in films of blue, green and red $\pi$-conjugated oligomers, each of them bearing complementary quadrupole hydrogen bonding ureidopyrimidone units (Fig. 7c) ${ }^{264}$ White electroluminescence was achieved with CIE (Commission Internationale de l'Energie) coordinates $(-0.31 ; 0.45)$ by using an appropriate ratio of the oligomers. Other examples of supramolecular polymers using hydrogen-bond interactions instead of metal complexation were also described for the fabrication of OLED devices. Chang et al. have reported the synthesis of $\pi$-conjugated poly(triphenylamine-carbazole) which exhibits amorphous nature, high thermal stability, good hole injection, and electron-blocking ability in the solid state owing to the supramolecular cross-linking of uracil units between polymer chains. ${ }^{265}$ Trilayer OLED devices based on tris(8-hydroxyquinolinato) aluminium $\mathrm{Alq}_{3}$ and the previous polymer as a hole injection/ transport layer have shown improved performance compared to PEDOT-PSS-based devices. Moreau and co-workers studied the capping of an oligophenylenevinylene imide derivative with 2,4-diamino-1,3,5-triazine derivatives by hydrogen bond interactions. $^{266}$ The resulting supramolecular complex led to J-aggregates showing increased luminance compared to the non-capped oligophenylenevinylene imide derivative.

Coordination complexes. Huang and co-workers have reported a novel spirometal platinium(II) complex which was applied for OLED devices. ${ }^{267}$ It exhibits a maximum luminance of $3335 \mathrm{~cd} \mathrm{~m}^{-2}$ and a maximum external quantum efficiency of $5.2 \%$. Iridium complexes have also attracted a lot of interest in this field and have been recently reviewed. ${ }^{256,258}$ Two examples of 
(a)

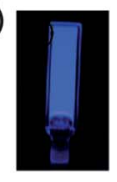

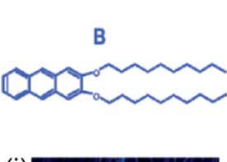

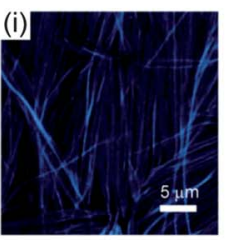

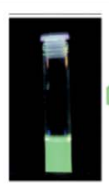
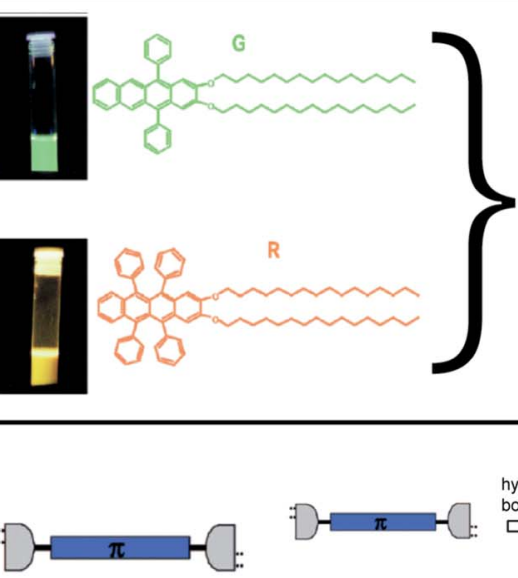

(c)

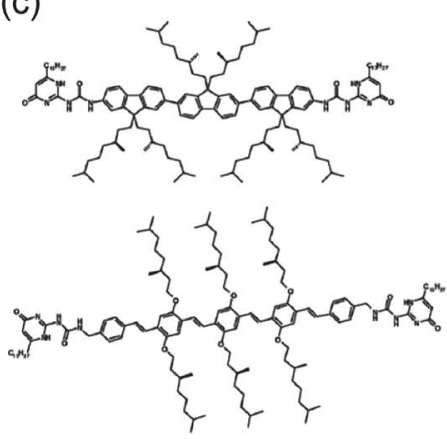
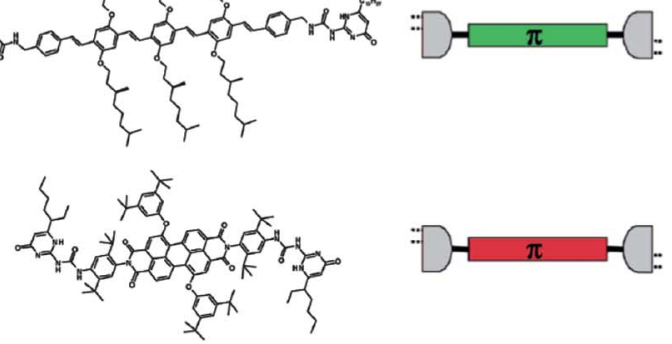
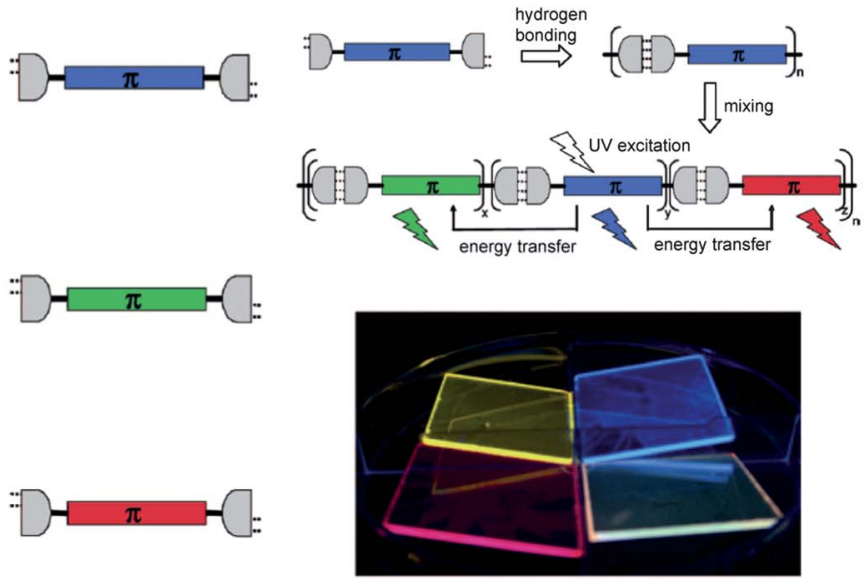

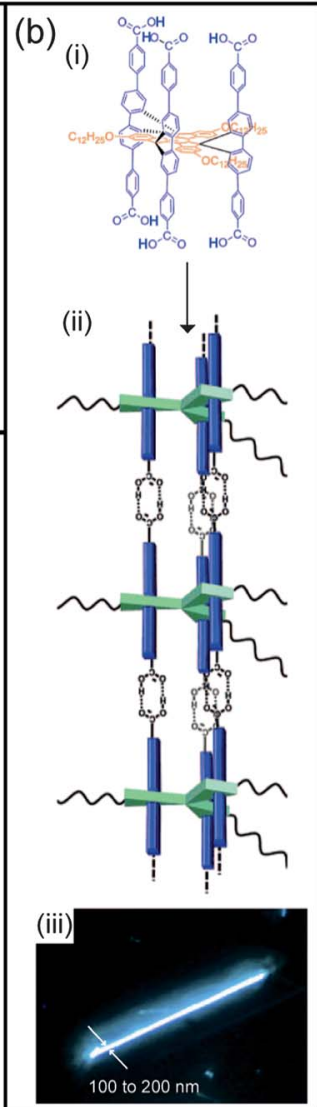

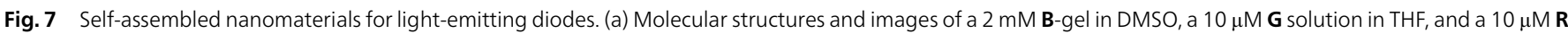

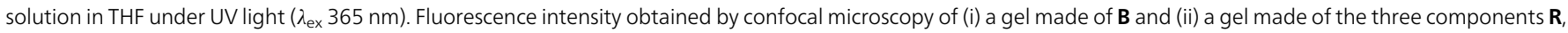

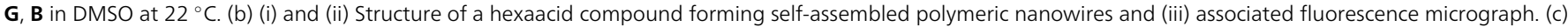

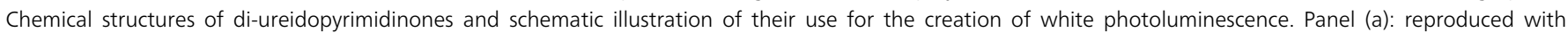
permission from ref. 260; (b) adapted with permission from ref. 262; (c) reproduced with permission from ref. 264.

the state of the art iridium-based devices are shown here. Bolink et al. reported an LEC device showing an extrapolated lifetime to half the initial luminance $\left(t_{1 / 2}\right)$ of 4000 hours (initial luminance $670 \mathrm{~cd} \mathrm{~m}^{-2}$ ), and sub-second turn-on times in applying a pulsed current driving method. ${ }^{268} \mathrm{Su}$ and co-workers described one of the white LEC devices showing the best performance to date with an external quantum efficiency (EQE) of $7.4 \%$ and a power efficiency of $15 \mathrm{~lm} \mathrm{~W}^{-1}$. $^{269}$

Inclusion complexes. Cacialli and co-workers have reported that energy transfer, from a blue emitting cyclodextrin threaded polymer to a green emitting one, is strongly reduced by controlling the minimum intermolecular distance between emitting moieties through non-covalent encapsulation of the blue component in the cyclodextrin macrocycles. ${ }^{270}$ The measured CIE coordinates $(0.282,0.336)$ correspond to warm light and a power efficiency of $0.39 \mathrm{~cd} \mathrm{~A}^{-1}$.

3.1.4 Supramolecular organic photovoltaic devices. Intensive research is currently being carried out in the field of organic photovoltaic devices, ${ }^{271-278}$ because of their lower cost compared to silicon-based materials and easy processing in devices. Morphology of the active layer plays a critical role in the device's performance and a lot of attention was devoted to its ordering. One way to achieve this organization is to use supramolecular materials capable of self-assembling in defined nanostructures. ${ }^{208,230-233,255,279-281}$

Nanofibers. Aida and co-workers have reported different selfassembly modes between enantiopure and racemate forms of porphyrin-fullerene dyads. ${ }^{282}$ The racemate generates nanometric spheres with a mean diameter of $300 \mathrm{~nm}$, whereas the enantiopure compound gives nanofibers up to $10 \mu \mathrm{m}$ in length. A cast film of the spherical assemblies shows only a small hole mobility of $1.5 \times 10^{-4} \mathrm{~cm}^{2} \mathrm{~V}^{-1} \mathrm{~s}^{-1}$ and no electron mobility by the time-of-flight method. ${ }^{248}$ Meanwhile, nanofibers exhibit hole and electron mobilities of 0.10 and $0.14 \mathrm{~cm}^{2} \mathrm{~V}^{-1} \mathrm{~s}^{-1}$, respectively. Shinkai et al. have described the self-assembly of electron rich quaterthiophene and electron poor perylene bisimide in order to obtain n-p heterojunction nodes (Fig. 8a). ${ }^{283}$ Interestingly, sorted formation of pure oligothiophene nanofibers and of pure perylene bismide nanofibers was observed from a $1: 1$ mixture of both compounds, and a bulk heterojunction solar cell was made using a cast film of this organogel. Nuckolls et al. have reported solar cell devices based on fullerene $\mathrm{C}_{60}$ and dibenzotetrathienocoronene (6-DBTTC), which self-assembles in a network of cables after thermal annealing. ${ }^{284}$ Devices, where the donor layer was annealed followed by vapor deposition of fullerene, show a power 


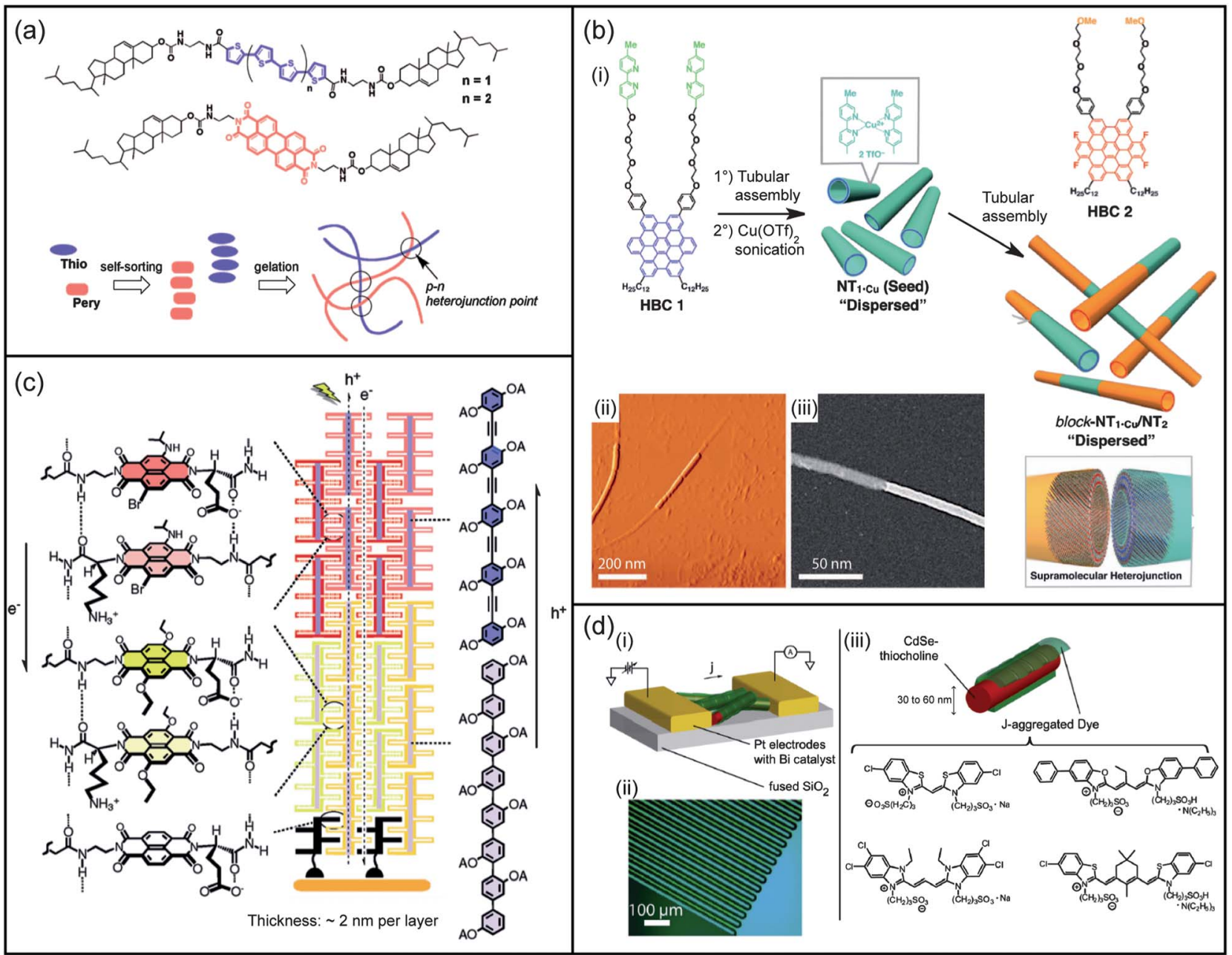

Fig. 8 Self-assembled nanomaterials for photovoltaic devices. (a) Chemical structures of the oligothiophene and perylene organogelators with a schematic representation of their self-sorting organogel formation yielding p-n heterojunction points. (b) (i) Molecular structures of two HBCs and schematic illustrations of the preparation of a supramolecular heterojunction made of semiconducting nanotubular segments; (ii) tapping-mode AFM and (iii) scanning TEM micrograph of the nanotubular segments. (c) Molecular structures of $p$-oligophenyl, $p$-oligophenylethynyl chromophores, and naphthalenediimides, together with their corresponding supramolecular heterojunction architectures resulting in oriented multicolored antiparallel redox gradients (OMARG). (d) Overview of a J-aggregate/CdSe nanowire color-selective photodetector; (i) schematic of J-aggregate-coated nanowires contacted between electrodes; (ii) optical micrograph, showing interdigitated electrodes used to grow and test devices. The faint brown shading between electrodes is the sparse network of CdSe nanowires; (iii) detail of J-aggregate-templated CdSe nanowires with the structure of J-aggregating dyes. Panel (a): reproduced with permission from ref. 283; panel (b): adapted with permission from ref. 290; panel (c): reproduced with permission from ref. 298; panel (d): reproduced with permission from ref. 301.

conversion efficiency of $1.9 \%\left(J_{\mathrm{SC}} 6.7 \mathrm{~mA} \mathrm{~cm}{ }^{-2} ; V_{\mathrm{OC}} 0.6 \mathrm{~V}\right)$. The same group has also reported solar devices based on blended films of 6-DBTTC and PC70BM. ${ }^{285}$ Supramolecular complexes are formed here in the bulk, and a $1: 2$ ratio of these two components gives the highest power conversion efficiency (PCE) of $2.7 \%$. Würthner and co-workers have described the use of triaminotriazine-sustituted oligo(phenylene vinylenes) to bind perylene bisimides in order to form hydrogen bonded trimers. ${ }^{286}$ The latter then self-assemble into right-handed supercoils. Organic photovoltaic (OPV) devices show only poor diode behavior due to the lateral organization of the supercoils at the surface. Holmes et al. have reported a polypeptide generating an alpha helix structure after gelation in an organic solvent. ${ }^{287}$ In solar cell devices, blends of the polypeptide and PCBM show an improvement of PCE compared to reference compounds which do not produce any helical arrangement of chromophores.

Nanotubes. Kamat and co-workers have used stacked-cup carbon nanotubes (SCCNTs) in a photoelectrochemical cell. ${ }^{288} \mathrm{~A}$ maximum photoconversion efficicency of $17 \%$ was reached that is lower than that of dye-sensitized solar cells but comparable to that of nanostructured semiconductor films $\left(\mathrm{TiO}_{2}, \mathrm{ZnO}\right.$, etc.). Aida et al. have synthesized photoconductive nanotubes by a controlled self-assembly of the HBC-trinitrofluorenone (TNF) covalent donor-acceptor dyad. ${ }^{289}$ Those nanotubes have a coaxial configuration in which a molecular layer of electronaccepting TNF laminates an electron-donating graphitic bilayer of $\pi$-stacked HBC. Such a structure creates an extremely wide interface for the spatially segregated redox couple, providing the electrical conduction an on-off ratio greater than $10^{4}$ upon 
photoirradiation. The same group has also reported a supramolecular linear $\mathrm{p}-\mathrm{n}$ junction composed of two different graphitic nanotubes by a stepwise self-assembly of two kinds of HBCs having different electronic structures (Fig. 8b). ${ }^{290}$ The lifetime of the charge carriers generated in the heterojunction nanotubes is five times longer than those measured for the nanotubes composed of a single component, demonstrating that the probability of the charge recombination is reduced. Hasobe and co-workers have also reported the use of a zinc meso-tetra(4-pyridyl)porphyrin to form hexagonal hollow nanotubes which were filled with $\mathrm{C}_{60}$ and $\mathrm{C}_{70}$ derivatives. ${ }^{291}$ Regenerative $\mathrm{I}^{-} / \mathrm{I}^{3-}$ photoelectrochemical cells made with these nanotubes show, at an input power of $20 \mathrm{~mW} \mathrm{~cm}^{-2}$, a PCE of $0.63 \%$ when the nanotubes are filled with a $\mathrm{C}_{60}$ derivative, and $0.03 \%$ when kept empty.

Liquid crystals. Wong and co-workers described the use of a difluorenyl-substituted HBC which self-assembled into columnar discotic liquid crystals. ${ }^{292}$ Solar cell devices based on the blend of the previous compounds with PC60BM $(1: 2)$ generated a short circuit current $\left(J_{\mathrm{SC}}\right)$ of $2.68 \mathrm{~mA} \mathrm{~cm}^{-2}$ and PCE of $1.46 \%$, after thermal annealing $\left(150{ }^{\circ} \mathrm{C}\right)$. The same authors also modified the latter compounds by attaching oligothiophenes. ${ }^{293}$ New devices based on blends of this compound and PC70BM $(1: 2)$ gave a $J_{\mathrm{SC}}$ of $2.53 \mathrm{~mA} \mathrm{~cm}^{-2}$ and PCE of $1 \%$. More recently, diketopyrrolopyrrole units were appended and blends with PC70BM showed a $J_{\mathrm{SC}}$ of $3.96 \mathrm{~mA} \mathrm{~cm}^{-2}$, a $V_{\text {OC }}$ of $0.86 \mathrm{~V}$ and PCE of 1.59\% ${ }^{294}$ A nematic liquid-crystal gel constituted of both a polymerizable electron donor and a non-polymerizable component was used by Kelly and co-workers. ${ }^{295}$ After photopolymerization and subsequent washing, an electron acceptor molecule layer was deposited to form a vertically separated distributed interface between donors and acceptors. Photovoltaic devices built from this method showed an open circuit voltage $V_{\mathrm{OC}}$ of $1.1 \mathrm{~V}$ and a power conversion efficiency of $0.6 \%$ for a monochromatic irradiance of $45 \mathrm{~mW} \mathrm{~cm}^{-2}$. Li et al. reported the incorporation of PCBM to liquid crystalline porphyrins which form columns perpendicular to the substrate. ${ }^{296,297}$ The resulting doped blend retained the liquid crystal phase of the porphyrin derivative, allowing the formation of highly ordered nanostructured thin films which are promising for OPV devices.

Zipper. Matile et al. have introduced the programmed assembly of interdigitating intra- and interlayer recognition motifs on conducting surfaces (Fig. 8c). ${ }^{298,299}$ These selfassembled zippers allow access to supramolecular coaxial $\mathrm{n}$-p-heterojunctions ${ }^{\mathbf{3 0 0}}$ with good photocurrents and high fill factors (0.50-0.60).

In addition to their interest for energy purpose, OPV devices could also be used as photodetectors by converting a light signal to an electrical one. Bawendi and co-workers reported the conception of photodetector devices based on the self-assembly of J-aggregate dyes onto crystalline inorganic nanowires, allowing the distribution of both light harvesting and charge transport functions between two materials optimized for each role (Fig. 8d). ${ }^{301}$ The deposition of strongly negative cyanine dyes by spin coating onto thiocholine-treated CdSe nanowires resulted in formation of J-aggregates at the nanowire surface. This process was shown to be reversible using exclusively solution phase processing and thus, a switchable color sensitization of the device from blue to red to green was explored. These colorsensitized devices demonstrated three specific and narrow absorption bands with a resulting photocurrent enhancement of up to 2.8-fold greater than the bare CdSe nanowire device. Samori and co-workers have also studied organic phototransistors based on perylene diimide (PDI) nanofibers. ${ }^{\mathbf{3 0 2}}$ The experiments show that the photoresponse can be modulated by tuning the device geometry and the morphology of the semiconducting layer. An optimized PDI multifiber-based device was then built from these considerations and a strong responsivity value of $4.08 \pm 1.65 \times 10^{5} \mathrm{~A} \mathrm{~W}^{-1}$ was obtained.

\subsection{Information storage and processing}

Extensive on-going research in molecular and supramolecular computing has been motivated by potential applications for information storage and/or processing which are of tremendous importance in our digital society. The following section has been divided into two parts: one focusing on self-assembled materials for non-linear optic applications, and the other on self-assembled Boolean logic gate materials.

3.2.1 Supramolecular materials for non-linear optics. Nonlinear optic (NLO) materials have applications in laser technology, optical communication, three-dimensional data storage, microscopy/imaging and microfabrication. ${ }^{303-307}$ Second-order NLO effects (e.g. for second harmonic generation) are usually observed in non-centrosymmetric materials either due to the molecular components or their arrangement in the condensed phase $;^{308}$ whereas third-order NLO effects (e.g. for two-photon absorption) ${ }^{309}$ are generally observed in centrosymmetric materials. Only few supramolecular self-assembled materials are described in the literature for non-linear optic applications and are reviewed hereafter.

Nanofibers. Ishi-i et al. have reported nanofibers based on hexaazatriphenylene-triphenylamine conjugates which show moderate improvement of the two-photon absorption crosssection compared to a non-aggregative analog. ${ }^{310}$

Inclusion complexes. The inclusion of (dimethylamino)stilbazolium-type chromophores in amylose helices was studied by Kuzyk and co-workers. ${ }^{311}$ Enhancement of the second-order nonlinear optical response was clearly observed through the inclusion compared to the free chromophores.

Coordination complexes. Burke et al. have described a dicopper(I) metallocyclophane with a partial $C_{2}$-symmetric structure in solution, allowing the observation of a strong second harmonic frequency response $\left(\beta=(3000 \pm 600) \times 10^{-30}\right.$ esu for a fundamental frequency at $800 \mathrm{~nm}) .{ }^{312}$ Kamada and co-workers reported the self-organization of two porphyrin-dimers through complexation allowing a strong two-photon absorption in the near-IR wavelength region. ${ }^{313}$ Finally, Kim et al. have shown that the formation of a double strand through coordination of bidendate ligands enhances the two-photon absorption crosssection of a conjugated porphyrin trimer, whereas the formation of a triple strand, by using a tridentate ligand, has the opposite effect. ${ }^{314}$ 
3.2.2 Supramolecular materials for information storage and computation. In this part, only examples of supramolecular self-assembled materials used as Boolean logic gates will be presented. Logic gate systems using supramolecular recognition only as inputs have not been included and recent reviews can be found elsewhere. ${ }^{315-317}$

Based on rotaxanes. Many rotaxanes in the literature could play the role of logic gates ${ }^{317-320}$ and we only review here those which were included in functional memory storage devices. Stoddart and co-workers have constructed a molecular memory circuit incorporating bistable [2] rotaxane molecules as the data storage element (Fig. 9a). ${ }^{321}$ This 160 000-bit molecular electronic memory circuit was fabricated at a density of 1011 bits per $\mathrm{cm}^{2}$ (pitch $33 \mathrm{~nm}$; memory cell size $0.0011 \mathrm{~mm}^{2}$ ). The working bits were then configured to form a fully functional random access memory circuit for storing and retrieving information. Gao et al. have designed a solid-state memory device based on a LangmuirBlodgett film made of amphiphilic bistable rotaxanes. ${ }^{322}$ The system exhibits electric bistability and nanometric dots (of $c a$. $3 \mathrm{~nm}$ size) can be written over the surface using a STM tip and erased by applying a reverse voltage pulse.

Based on self-replicating systems. Non-enzymatic reaction networks were used by Ashkenazy and coworkers to design light-triggered logic gates. ${ }^{323}$ First, a coiled-coil peptide able to catalyse its replication was caged with a photocleavable moiety preventing the autocatalytic pathway from occurring. ${ }^{324}$ Lightinduced uncaging of the latter allows restoration of the selfreplicative system and thus shows an AND gate behaviour with both light and the caged peptide as entries. A couple of FRET donor-acceptor moieties was then added to this system. ${ }^{325}$ Hence, fluorescence quenching of the mixture can be used to monitor the replication process. Considering fluorescence as the output signal of the logic gate, the latter becomes a lightinduced NAND gate.

Based on DNA. A lot of attention has also been devoted to biocomputation with DNA. ${ }^{326-329}$ Winfree et al. have designed
DNA-based logic circuits ${ }^{330}$ producing AND, OR, NOT gates, as well as signal restoration, amplification, feedback, cascading, and even a circuit able to compute the integer part of the square root of a four-bit binary number. ${ }^{331}$ These systems rely on sequence recognition and nucleic base displacement by using only single stranded DNA as inputs and outputs. Also resting on simple strand displacement, Ghadiri and co-workers have described the serial connection of DNA-based logic gates in the same solution by using fluorescence as the output. ${ }^{332} \mathrm{~A}$ halfadder was obtained from an AND gate and an XOR gate, while an OR gate was assembled through two XOR gates. DNA structures like hairpins have also been used to build logic gates. Park et al. have designed a set of two-input logic gates based on a hairpin-structure molecular probe. ${ }^{333}$ These gates can be reset by applying an additional input or a removal strand. Turberfield and co-workers have reported another DNA hairpin-based AND gate which is able to continuously re-compute its output, thus changing its response to various inputs. ${ }^{334}$ DNA sensors based on hairpin structures equipped with redox-tags and immobilized on electrode surfaces have also been reported. ${ }^{335,336}$ Another approach to design DNA-based logic gates is to use DNA molecules with biocatalytic properties mimicking enzyme functions, so-called DNAzymes. ${ }^{337}$

Willner et al. have designed an entire DNA computing circuit based on the modular coupling of DNAzyme subunits. ${ }^{338}$ This system could be tailored to generate multilayered gate cascades, fan-out gates, or parallel logic-gate applications. Other groups have reported logic gates based on ion-dependent DNAzymes. ${ }^{339-341}$ Ion-tuned DNA/Ag nanoclusters ${ }^{342}$ can also be used for the design of versatile logic gates as shown by Wang, Dong and co-workers (Fig. 9b). ${ }^{343}$ Inputs $\left(\mathrm{K}^{+}\right.$and $\mathrm{H}^{+}$ions) induce changes of the DNA stabilizer which result in modifications of the spectral behaviour of Ag nanoclusters. Thus, multiple logic operations can be performed together via multichannel fluorescence output. Finally, Fan and co-workers designed tetrahedral DNA nanostructures as logic gates (AND, OR, XOR, INH,

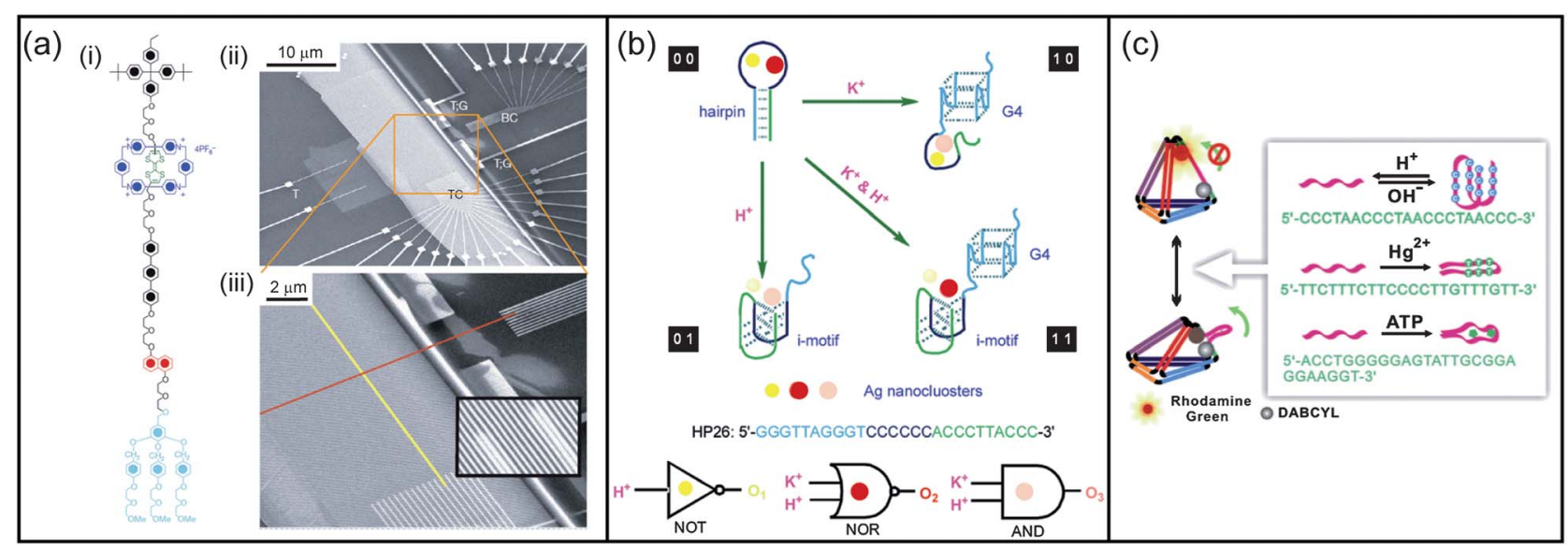

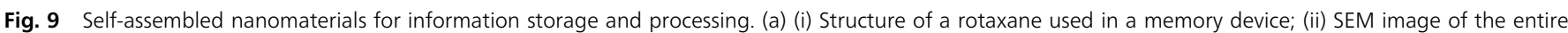

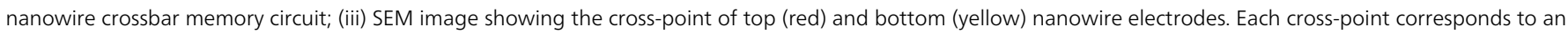

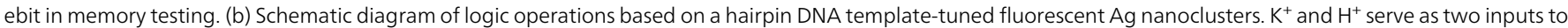

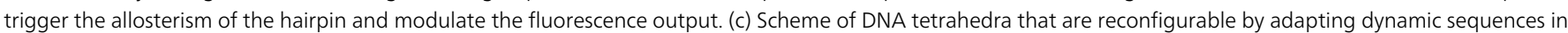

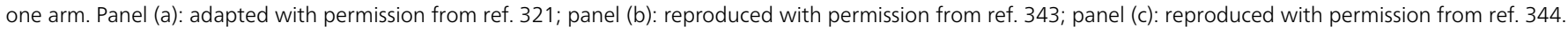


and a half-adder). ${ }^{344}$ These are responsive to protons, $\mathrm{Hg}^{2+}$ ions, ATP, and complementary DNA scaffolds (Fig. 9c). An example of a tetrahedra logic gate was also tested for intracellular detection of ATP in living cells.

These self-assembled materials are promising logic gates, especially DNA which could have exceptional storage capacities. ${ }^{345,346}$ However, for most of the examples in the literature, issues concerning the ways and means to integrate them in practical devices should be addressed to advance further in the field.

\section{Self-assembled nanomaterials for environmental sciences}

One of the main societal issues of the century concerns environment and in particular how to solve problems related to pollution and wastes. Various approaches ranging from inorganic to polymeric or carbon materials have been envisioned to overcome these issues. ${ }^{347}$ While still in their infancy, functional devices made of supramolecular self-assemblies have emerged as promising tools to tackle some of these environmental concerns. ${ }^{348}$ In this chapter, we will illustrate the variety of nanostructured objects produced by self-assembly, or involving a molecular recognition event to operate, that have been used to address these problems..$^{349-351}$ In the first part, recent selected examples will highlight the various self-assembled structures used to sense chemical analytes ranging from gases to metal ions up to substances present in food or cosmetics. ${ }^{352-355}$ In the second section, supramolecular materials that are used to store gases or small molecules will be described, in particular nanoporous molecular crystals and metal organic frameworks. ${ }^{350,351,356}$ In the third part, we will first point out the importance of supramolecular ion channels as synthetic transport systems and further demonstrate the importance of nanostructured membranes and metal-organic frameworks for separation and purification of mixtures of gases or chemicals. ${ }^{350,351,357-360}$ Finally, we will show the wide diversity of nanometric objects that have been involved in the catalysis of chemical reactions. ${ }^{350,351,361-363}$

\subsection{Nanomaterials for detection}

In recent years, many efforts have been devoted to the development of (bio)chemical sensors using the supramolecular approach. ${ }^{364-369}$ Although a large number of examples rely on the host-guest recognition of small molecules for sensing analytes, ${ }^{370-372}$ we will focus here on systems that are considered as nanomaterials, i.e. made of supramolecular self-assemblies: ribbons, pores, gels, arrays, nanoparticles, quantum dots, and metal-organic frameworks.

Supramolecular gels. Almost ten years ago, with the aim to retain chemosensors' activities upon immobilization, the group of Hamachi described the first supramolecular hydrogel as an efficient platform for chemical sensing. ${ }^{373,374}$ Interestingly, this methodology is not limited to the sensing of metal cations ${ }^{373}$ or polyanions ${ }^{374}$ but is also applicable to enzymes and ligands such as lysylendopeptidase, chymotrypsin, or glycoconjugates. ${ }^{375}$
More recently, the same authors have shown that a hydrogel hybrid, made of supramolecular fibers $\mathbf{1}$ and coumarin dyes adsorbed on montmorilllonite (G-coum $\subset \mathrm{MMT}$ ), is able to detect polyamines such as spermine or spermidine from an artificial urine medium in the $0-50 \mu \mathrm{M}$ range concentration (Fig. 10a). ${ }^{376,377}$ Upon exposure to cationic amines, the guanidinium-functionalized coumarin is released from the clay and subsequently translocated to the supramolecular hydrogel. Importantly, the fluorescence of the coumarin released upon addition of spermine is blue-shifted in comparison to G-coum $\subset$ MMT (from $540 \mathrm{~nm}$ to $470 \mathrm{~nm}$ upon excitation at $365 \mathrm{~nm}$ ), which is easily detectable by fluorocolorimetric imaging of a hybrid sensor array chip. Therefore, this system can be used as a rapid naked-eye detector for polycationic amines, and the fluorescence intensity ratio could help in quantifying the analyte. In 2012, the same group has extended its methodology from a semi-wet environment to a dry one by sensing polyols on a paper platform, using FRET as the signaling event. ${ }^{378}$ Other groups have also reported the use of hydrogels based on polyacrylamide polymers decorated with side-chain DNA strands as a colorimetric platform for the detection of cocaine and mercury. ${ }^{379,380}$ In the first case, a gel-sol process induced by the formation of an aptamer has been used to probe the presence of cocaine. ${ }^{379}$ The addition of a colorimetric probe allows for the visual detection of quantities of cocaine as little as $100 \mathrm{ng}$, and no interferences from cocaine metabolites were observed. In the second example, a DNA hairpin was formed in the presence of mercury, leading to green fluorescence upon exposure to a fluorescent probe. ${ }^{380}$ This gel was shown to be selective to mercury and could be used at concentrations as low as the toxic level in drinking water $(10 \mathrm{nM})$. Recently, the group of Kimura reported the use of supramolecular gel membranes made of low-molecular weight gelators to sense alkali ions with a very good selectivity for potassium. ${ }^{381}$

Graphene and nanoparticles. In 2009, the group of Shi reported the use of a graphene-porphyrin complex as an optical sensor for the selective detection of cadmium ions in aqueous media. ${ }^{382}$ Plasmonic resonance energy transfer (PRET) on gold nanoplasmonic probes was also used to selectively detect copper over other metal ions with a high spatial resolution and at low concentrations. ${ }^{383}$ However, most of the sensors developed so far using nanoparticles also involve DNA strands as the recognition units, as detailed below.

DNA-based sensors. Simple optical sensors - such as a thymine-rich DNA strand functionalized at its $3^{\prime}$ and $5^{\prime}$ termini by FRET probes - have been designed for the selective sensing of mercury (hairpin formation) and lead (aptamer formation) in environmental samples. ${ }^{384}$ However, DNA-based sensors usually take advantage of nanoparticles for achieving detection at low nanomolar concentrations. Hybrid DNA-gold nanoparticles have also been widely used for mercury and lead detection, reaching nanomolar detection limits. ${ }^{385,386}$ The group of Lu has used a DNAzyme specifically cleaved by lead ions, thereby generating ssDNAs that prevent aggregation of the gold nanoparticles and thus lead to a red colored solution different from the one observed when the nanoparticles are not aggregated, i.e. when the DNAzyme is not cleaved. ${ }^{386}$ Mirkin and coworkers have 


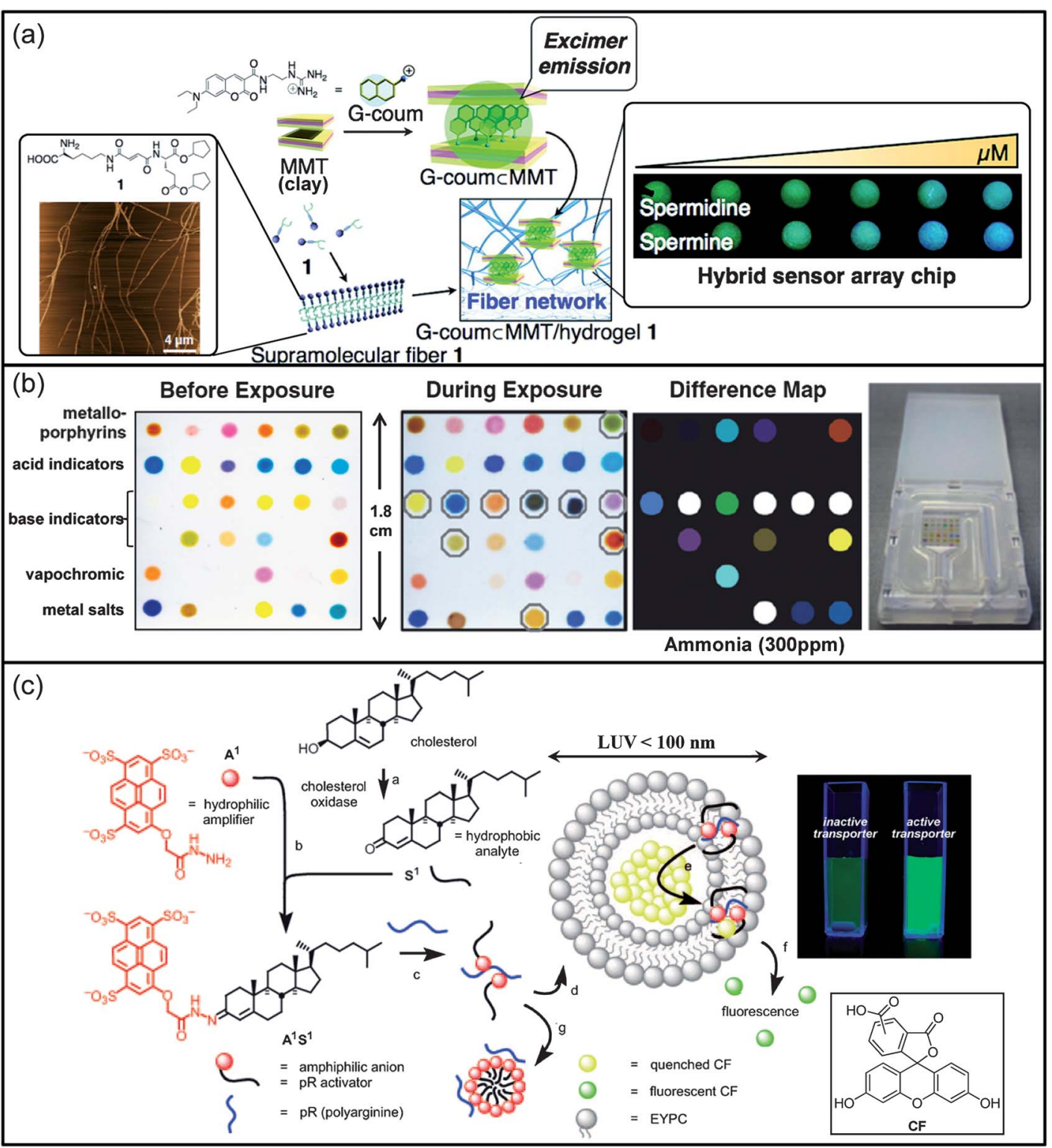

Fig. 10 Self-assembled nanomaterials for detection. (a) Construction and mechanism of action of a fluorescent dye (G-coum) adsorbed on a MMT (montmorillonite)/ supramolecular hydrogel $\mathbf{1}$ hybrid sensor system for polyamines; inset left: chemical structure of amino acid hydrogelator $\mathbf{1}$ and atomic force microscopy image of hydrogel 1 ([1] = $1.0 \mathrm{wt} \%$ /ion-exchanged water); inset right: photograph of the G-coum $\subset$ MMT/hydrogel 1 hybrid sensor chip $\left(\lambda_{\mathrm{ex}}=365 \mathrm{~nm}\right)$ for fluorocolorimetric sensing of spermidine and spermine in artificial urine. (b) Colorimetric sensor array consisting of 36 different chemically responsive pigments which have been printed on a nonporous polyethylene terephthalate (PET) film, and images of the 36-dye colorimetric sensor array before exposure and after 2 min of exposure to ammonia at its IDLH (immediately dangerous to life or health) concentration at $298 \mathrm{~K}$ and $50 \%$ relatively humidity, and the generated color difference map; right: disposable low dead-volume self-sealing cartridge with the colorimetric sensor array printed directly on a PET flat. (c) Fluorogenic steroid sensing systems with cell-penetrating peptide (CPP) transporters as signal transducers and signal amplifiers (e.g., $\mathbf{A}^{\mathbf{1}}$ ) that react with hydrophobic analytes (e.g., $\mathbf{S}^{\mathbf{1}}$ ) to generate amphiphilic CPP activators. For cholesterol, signal generation with cholesterol oxidase (a) is followed by signal amplification with $\mathbf{A}^{\mathbf{1}}$ (b). The obtained $\mathbf{A}^{\mathbf{1}} \mathbf{S}^{\mathbf{1}}$ conjugate and CPP (e.g., pR (polyarginine)) form $p R-\mathbf{A}^{\mathbf{1}} \mathbf{S}^{\mathbf{1}}$ transporters (c) that mediate the export of intravesicular 5(6)-carboxyfluorescein CF (d-f). Amphiphilic anions (e.g., $\left.\mathbf{A}^{\mathbf{1}} \mathbf{S}^{\mathbf{1}}\right)$ are CPP activators below (d-f) and CPP inactivators above their $\mathrm{cmc}(\mathrm{g})$; right: detection of active transporters as fluorescence recovery with CF-loaded vesicles. Panel (a): adapted with permission from ref. 376 and 377; (b) adapted with permission from ref. 367; (c) reproduced with permission from ref. 365 and 403.

shown that gold nanoparticles modified with complementary thymidine-containing DNA strands can serve as colorimetric detectors for mercury by displaying a much higher melting temperature than when sensing other metals. ${ }^{385}$ The use of thymidine- $\mathrm{Hg}^{2+}$-thymidine coordination complexes for detecting mercury is however not only limited to solution as it was shown by the detection of heavy metals using microarrays. ${ }^{387}$ In this case, polydiacetylene liposomes bearing a thymine-rich ssDNA aptamer as side chains were affected by the steric hindrance induced by the mercury complexes, leading to a change in the fluorescence color (blue to red) with a detection limited to the micromolar range.

Arrays. In general, arrays have been widely developed for sensing single analytes but, more recently, they have also been 
used for sensing complex mixtures. ${ }^{354,388}$ Among them, Suslick and coworkers have shown that colorimetric sensor arrays made of well-defined chemically responsive dyes can be suitable for analyzing various compounds ranging from toxic gases to sweeteners or even bacteria (Fig. 10b).$^{389-391}$ In order to obtain a robust device that could sense both liquids and gases, colorants were chosen in accordance with their ability to chemically interact or react with the analytes (that is beyond simple physisorption) and nanoporous pigments were chosen to avoid leaching in solution. The difference map obtained by comparing the changes in color before and during exposure to the analyte allows its qualitative recognition by naked eye and, in addition, quantitative analysis could be performed using standard chemometric techniques such as hierarchical cluster analysis (HCA) or principal component analysis (PCA). ${ }^{392}$ These mathematical approaches have also been used by the group of Anslyn and Severin for deciphering sensor arrays and/or dynamic combinatorial libraries made of metal-ligand complexes. ${ }^{393,394}$ By monitoring the changes in absorbance of a peptidic array, Anslyn has shown that flavonoids and more generally red wines could be identified by an indicator displacement array (IDA). ${ }^{395,396}$ In a similar manner, by following the fluorescence of dyes displaced upon addition of the analyte, Severin has shown that two similar dipeptides present in a deproteinized human blood serum can be identified and quantified..$^{397}$

Dynamic combinatorial libraries. Although sensing using a dynamic combinatorial library (DCL) was first described by Lehn and Giuseppone, ${ }^{398}$ a major contribution to this emerging field has come from the group of Severin. ${ }^{399,400}$ The main difference between sensing with a classical array and with a DCL is that only a single optical measurement is necessary for determining and quantifying the analyte. In particular, it has been shown that DCL can rival cross-reactive arrays for sensing di- and tripeptides. ${ }^{401}$ More recently, Matile and Takeuchi have shown that differential sensing of aldehydes and ketones can be achieved using synthetic transport systems. ${ }^{365}$ Their methodology is established on the ability of DNA or cell penetrating peptides (CPP) such as polyarginine to bind and transport ionic amphiphiles into bilayer membranes, which then assist the transport of small hydrophilic fluorescent anions across bilayer membranes (Fig. 10c). For example, fragrant aldehydes and ketones were sensed very efficiently using reactive hydrazide cations to generate amphiphilic hydrazones that can activate DNA to enter lipid bilayer membranes. ${ }^{402}$ Samples from daily life such as commercially available perfumes were also efficiently distinguished by this approach. Interestingly, when no aldehyde or ketone was present on the analyte of interest, the use of an enzyme as the signal generator was particularly attractive. This approach was successfully applied to the sensing of steroids even at low micromolar concentrations. ${ }^{403}$ The high selectivity of enzymes allowed cholestrerol sensing in real-life samples such as egg yolk or human blood serum. It is noteworthy that this approach was the first example in which a CPP-based sensor is superior to synthetic pores.

Nanopores. The group of Matile also demonstrated some years ago that artificial $\beta$-barrels (or nanopores) composed of four $p$-octiphenyl molecules held together by short $\beta$-sheetsforming peptides and inserted into unilamellar vesicles could serve as artificial tongues for sensing a wide variety of substrates in complex matrices. ${ }^{404}$ After a suitable preparation of the sample to avoid interferences, the analytes of interest were enzymatically modified and, for the poorly recognized ones, were further reacted with a signal amplifier in order to block the pore, thereby producing fluorescence self-quenching of the vesicle-loaded fluorophores. This methodology has been successfully applied to sensing of components of real food mixtures. ${ }^{405-407}$

Nanoribbons. In 2010, the groups of Zhao and Zang reported the use of 5 nanometer-thick nanoribbons based on a donoracceptor supramolecule to sense nitro-based explosives with varying vapor pressures as well as some of the common organic solvents. ${ }^{408}$ By monitoring the conductivity of the system, which is reduced upon exposure to volatiles probably because of the depletion of the charge carriers upon absorption, 2,4-dinitrotoluene could be detected at a concentration as low as $0.1 \mathrm{ppm}$. Interestingly, this supramolecular system displays a very quick recovery after exposure to volatiles, which in principle could allow a high throughput screening of these explosives.

MOFs. In recent years, metal-organic frameworks (MOFs) have been widely used for sensing analytes ranging from solvents, gases, water, chiral and volatile organic molecules up to nucleotides. ${ }^{36,409-417}$ Just to mention one example, a film made of ZIF-8 has been used for the detection of methanol vapor without any further labeling, as its exposure to the analyte induces a green-to-red color change visible to the naked eye. ${ }^{409}$ Its potential recycling has been also demonstrated by the removal of methanol under vacuum which leads to the recovery of the green film. Interestingly, MOFs do not only serve as sensors but, in most cases, also as materials for chemical storage (see Section 4.2).

Host-guest devices. To conclude this section, some devices based on host-guest recognition deserve to be mentioned as they might open routes for applications. For example, Dalcanale and coworkers have shown that microcantilevers and carbon nanotubes functionalized with cavitand receptors can be used without any labeling to detect $N$-methylammonium compounds, which are well-known as drugs, cancer biomarkers and neurotransmitters. ${ }^{418,419}$ Another interesting device is the use of a hybrid protein-carbon nanotube field effect transistor to differentiate bitter and non-bitter tastants at femtomolar concentrations. ${ }^{\mathbf{4 2 0}}$

\subsection{Nanomaterials for chemical storage}

One classical feature of nanomaterials used in chemical storage is their structuration around nanopores, but we will see below that they are not restricted to this characteristic. The particular topic of the absorption of guest molecules on surfaces made of 2-D self-assembled nanopores will not be discussed here, although they can be used for gas storage, and readers should refer to reviews recently published in the literature. ${ }^{\mathbf{4 2 1 , 4 2 2}}$

Dynamic combinatorial libraries. One example of a system that does not involve a nanoporous cavity rests on the use of a DCL to store carbon dioxide. ${ }^{423}$ In that case, the authors took 
advantage of the reversible nature of both the imine bond and $\mathrm{CO}_{2}$ capture by ammonium carbamate formation to induce a precipitate presenting a mass content in $\mathrm{CO}_{2}$ of $20 \%$. Interestingly, only the adduct containing $\mathrm{CO}_{2}$ precipitated, which might allow its separation from other gases, and more than 95\% desorption was achieved while heating the structure at around $100{ }^{\circ} \mathrm{C}$.

Non-porous crystalline solids. Another example is the use of self-assembled bis-urea pyridyl macrocycles that form a onedimensional crystalline solid without pores. ${ }^{\mathbf{4 2 4}}$ Soaked or vapors of alcohols have been absorbed due to their hydrogen bonding ability with the urea oxygen lone pairs, and pentafluoroiodobenzene (which possesses an electrophilic iodide) has been stored and further released from this organic crystal.

Nanoporous molecular crystals. Using very similar bis-urea macrocyles, the same group was able to produce some thin needle-like porous crystals with an average size of $\sim 250 \mu \mathrm{m} \times$ $3 \mu \mathrm{m}$, as demonstrated by SEM images (Fig. 11a). ${ }^{425}$ Interestingly, these nanoporous fibers could only be formed in the presence of the host, namely acetic acid, and different assembly patterns were observed when building the self-assembly with other carboxylic acids. In all cases, as demonstrated by X-ray powder diffraction (XRPD), adsorption of the guest was also shown to be reversible thus leading to apohosts with the same structure whatever the guest was. Other molecules such as $p$-xylene or carbon dioxide were shown to be reversibly absorbed by any apohost preformed using a different guest molecule. These self-assemblies, so-called nanoporous molecular crystals, are very important for gas storage and significant improvements have been observed over the last few years. ${ }^{\mathbf{4 2 6 - 4 2 9}}$ In $2007,1,2-$ dimethoxy-p-tert-butylcalix[4]dihydroquinone was shown to self-assemble by taking advantage of hydrogen bonding and van der Waals interactions to produce polar open channels and two hydrophobic cavities which selectively absorb carbon dioxide over hydrogen at 20 atm. ${ }^{\mathbf{4 3 0}}$ In further work, the authors have also reported the absorption of methane, argon, and acetylene within the nanochannels, as confirmed by XRPD. ${ }^{431,432}$ Further efforts have then been two-fold: (i) increasing the BET (Brunauer, Emmett and Teller) surface area for improving the absorption capacities and/or (ii) ameliorating the selectivity of absorption. In 2010, the groups of Champness and Schröder demonstrated the high thermal stability of a 3D supramolecular organic framework made of polypyridyl tectons and its high potential for ethylene uptake and $\mathrm{CO}_{2}$ removal from the air. ${ }^{433}$ The same year, McKeown and coworkers synthesized a phthalocyanine-based nanoporous molecular crystal displaying the highest surface area $\left(1002 \mathrm{~m}^{2} \mathrm{~g}^{-1}\right)$, total pore volume, and pore size at that time. This crystal presents many similarities to the way hemoproteins function. ${ }^{\mathbf{4 3 4}}$ One year later, the group of Chen reported a hydrogen-bonded organic framework based on 2,4-diaminotriazine moieties that exhibits high thermal stability with a moderate surface area $\left(\sim 360 \mathrm{~m}^{2} \mathrm{~g}^{-1}\right)$ but with an exceptional selectivity for the separation of ethylene over ethane at ambient temperature. ${ }^{\mathbf{4 3 5}}$ Very recently, the group of Mastalerz reported the self-assembly of triptycenetrisbenzimidazolone as a porous crystal with one dimensional channels that displays the highest BET surface area reported to date for porous materials made of discrete molecules and that shows high absorption of $\mathrm{H}_{2}$ and selectivity for $\mathrm{CO}_{2}$ over methane, with values that compete with the best performing MOFs. ${ }^{436}$ One strategy for obtaining porous organic materials is the use of organic molecules presenting defined cavities (such as cage compounds) therefore displaying an intrinsic volume for storage.

In 2009, the group of Cooper reported on the synthesis of porous organic cages via $[4+6]$ cycloimination between $1,3,5$ triformylbenzene and various 1,2-diamines that further assemble in microporous organic materials (one example is displayed in Fig. 11b). ${ }^{437}$ All cages were shown to display gas sorption properties with BET surface area comprised between 530 and $620 \mathrm{~m}^{2} \mathrm{~g}^{-1}$. The same group then demonstrated that one of their organic cages can present different polymorphs depending on a chemical trigger, i.e. the solvent used for crystallization. ${ }^{438}$ Interestingly, these different polymorphs display different gas sorption properties that permit the selective sorption of hydrogen over nitrogen following a reversible on-off switching process. Cooper and co-workers further showed that the structure of their porous materials can indeed be predictable by using in silico simulations, leading to the discovery of the largest organic chiral cage molecule with the highest surface area observed for a material tackle by using DFT calculations. ${ }^{439}$ Based on this work, in which the authors have shown that the mixing of both CC3- $\boldsymbol{R}$ and $\mathbf{C C 3}-\boldsymbol{S}$ results in the formation of a racemic precipitate as a result of chiral recognition, ${ }^{\mathbf{4 4 0}}$ they were able to enhance the surface area and the porosity of macroporous inorganic supports such as silica and alumina-silica beads (Fig. 11b), thereby presenting potential interest for further applications in chromatography or catalysis (see Sections 4.3 and 4.4$){ }^{441}$ In parallel to these achievements, the group of Mastalerz has developed a series of salicylbisimine cage compounds which present very attractive properties for gas storage (Fig. 11c). ${ }^{429}$ First of all, they have shown that the cage compound depicted in Fig. 11c displays a very high BET surface area $\left(1377 \mathrm{~m}^{2} \mathrm{~g}^{-1}\right)$ which can be improved up to $2071 \mathrm{~m}^{2} \mathrm{~g}^{-1}$ after crystallization with a suitable solvent, yielding the highest surface area observed for cage compounds. ${ }^{\mathbf{4 4 2 , 4 4 3}}$ Interestingly, when the imine bonds were reduced to amine ones, the cage compound completely lost its gas sorption property, indicating the importance of the rigidity of the structure for gas absorption. From an applicative point of view, films made of these cage compounds were deposited on the electrode surface of a quartz crystal microbalance (QCM) and could be used as sensors for common aromatic solvents. ${ }^{44}$ To complete this study, the authors have shown that the size and the shape of the cavity has a great influence on the selectivity of binding, and that $[2+3]$ exo-functionalized cage compounds outperform "state-of-theart" compounds (modified cyclodextrins) used as high affinity materials for QCMs.

Peptidic gels. Another strategy for building nanostructures with porosity and gas sorption properties is to use peptidic residues such as cyclic peptide nanotubes or dipeptides. ${ }^{\mathbf{4 5}}$ For example, dialanine was shown to selectively absorb oxygen over nitrogen or argon with high selectivity, the other gases not even being detected. ${ }^{\mathbf{4 4 6}}$ Recently, the group of Haldar has shown that 

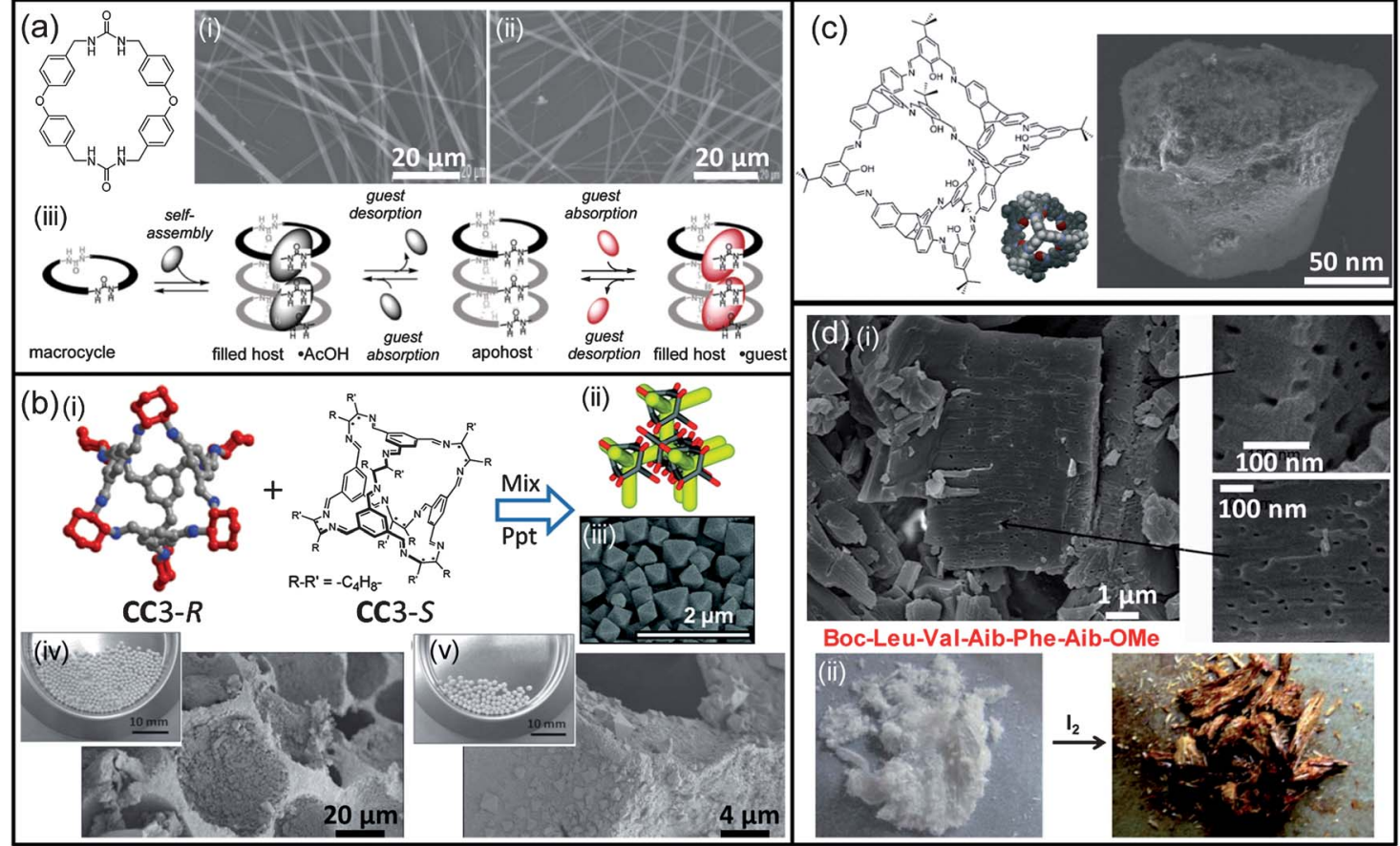

Boc-Leu-Val-Aib-Phe-Aib-OMe
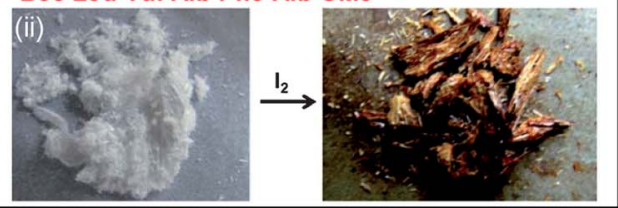

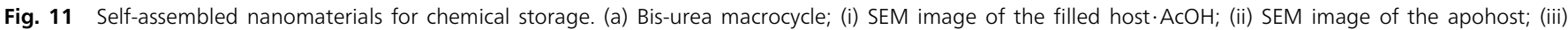

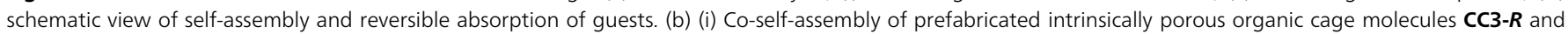

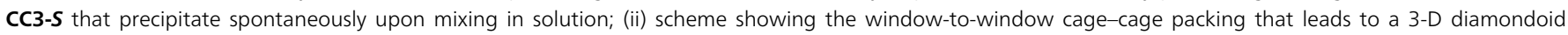

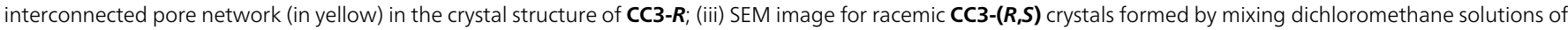

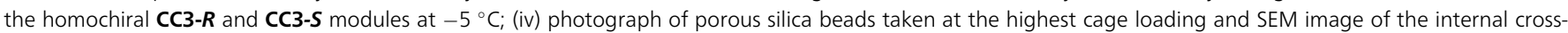

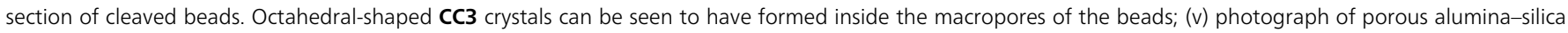

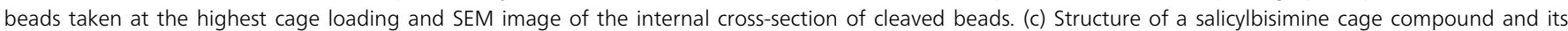

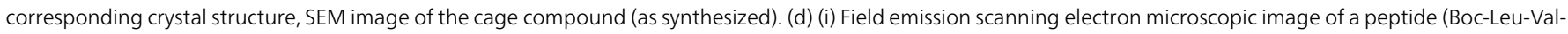

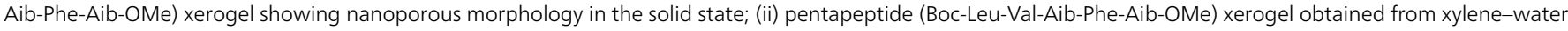

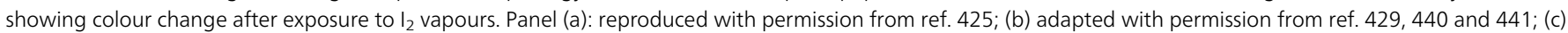
reproduced with permission from ref. 442; (d) reproduced with permission from ref. 447.

a hydrophobic pentapeptide displays a gel-type behavior in xylene-water, with a nanoporous morphology in the solid state, whereas a non-porous material is obtained from pure xylene (Fig. 11d). ${ }^{447}$ The xerogel was then used to absorb iodine vapors as shown in Fig. 11d(ii) and displayed efficient dye removal (crystal violet, rhodamine) from waste water. However, one major achievement using the peptidic residue is their incorporation in MOFs. ${ }^{\mathbf{4 4 8 , 4 4 9}}$

MOFs. Zinc-dipeptide frameworks were shown to be selective for polar molecules such as $\mathrm{CO}_{2}$, methanol, or water due to their polar interactions with the amide bonds, however with BET surface areas that do not reach the standard observed in MOFs. Indeed, systems with surface areas exceeding $5000 \mathrm{~m}^{2} \mathrm{~g}^{-1}$ have been recently achieved, ${ }^{\mathbf{4 0 4 , 4 5 0 , 4 5 1}}$ the highest one being of $7140 \mathrm{~m}^{2} \mathrm{~g}^{-1}$ but with a maximum theoretical upper limit of $14600 \mathrm{~m}^{2} \mathrm{~g}^{-1} .^{452}$ Interestingly, along their very high surface areas, MOFs have been shown to display excellent storage capacity for gases of importance in environmental sciences such as methane, hydrogen or carbon dioxide. ${ }^{453-457}$ For example, $\mathrm{CO}_{2}$ could be captured and recycled several times from air and flue gas with a $\mathrm{CO}_{2}$ purity exceeding $96 \%$, and without losing the MOF capacity upon recycling. ${ }^{458}$ In another example, a partially interpenetrated MOF was used to capture gases such as $\mathrm{CO}_{2}, \mathrm{~N}_{2}, \mathrm{CH}_{4}, \mathrm{O}_{2}, \mathrm{Ar}$, and $\mathrm{H}_{2}$ at high pressure and to further store $\mathrm{CO}_{2}$ trapped in the nanopores when decreasing the pressure ${ }^{459}$ MOFs have also been applied to selective sorption of organic vapors such as methanol, acetonitrile or benzene over water, thus presenting a potential interest for water purification. ${ }^{\mathbf{4 6 0}}$ Recently, the group of Kitagawa reported one of the rare examples in which $\mathrm{O}_{2}$ and NO were selectively absorbed over other atmospheric gases by a MOF due to charge transfer interactions between the guests and the organic linkers of the MOF. ${ }^{461}$ Finally, a recent advancement in $\mathrm{CO}_{2}$ storage is the use of flexible MOFs that are not only used to absorb $\mathrm{CO}_{2}$ over other atmospheric gases but also to confirm its presence by a significant change in fluorescence due to the presence of a reporter molecule within the MOF. ${ }^{\mathbf{4 6 2}}$ Interestingly, the fact that MOFs are able to selectively capture one gas in a mixture makes them suitable for separation and purification purposes. ${ }^{455}$

\subsection{Nanomaterials for separation/purification and transport}

\subsubsection{Synthetic transport systems}

Nanopores and nanochannels. The advanced field of synthetic transport systems rests mainly on the use of nanochannels and nanopores for transporting ions, gases, or small molecules 
across bilayer membranes. ${ }^{463}$ Of particular interest is the work by Chui and Fyles, who have recently correlated the topology of synthetic ion channels with their class of activity, their conductance, and their opening duration, demonstrating the broad diversity in terms of structures. ${ }^{\mathbf{4 6 4}}$ For example, the use of cholate to produce efficient ion transporters is well documented; particularly using cholapods and oligocholates. ${ }^{463,465}$ Zhao and coworkers have lately demonstrated the formation of transmembrane nanopores using four amphiphilic tri- or tetraoligocholate macrocycles through hydrophobic interactions, following a highly cooperative mechanism. ${ }^{466}$ Counter-intuitively, this system showed a more efficient transport of hydrophilic molecules in an increased hydrophobic environment and a faster transport of longer sugars versus shorter ones in a larger pore size. Recently, the group of Hou revealed new structures based on pillar[5] arenes for the efficient transport of water across bilayer membranes and demonstrated that fine tuning of the structure can block the transport of protons, like in natural water channel proteins. ${ }^{\mathbf{4 6 7 , 4 6 8}}$ Another important characteristic of the recently developed nanochannels is their responsiveness to the environment. In 2006, the group of Futaki developed an artificial peptidic channel that displays ion fluxes only in the presence of $\mathrm{Fe}(\mathrm{III})$ ions and can be reversibly closed by adding EDTA as a competing ligand, thereby preventing the destabilization of the extra-membranar helical structure of the peptide, which causes the opening of the pore. ${ }^{469}$ In 2008 , the groups of Satake and Kobuke took advantage of electrostatic interactions between carboxylates present at the outer side of a large artificial pore made of self-assembled oligoporphyrins and polycationic dendrimers to block a channel under low $\mathrm{pH}$ and allow its reopening when increasing the $\mathrm{pH}$ or the concentration of buffer. ${ }^{\mathbf{4 7 0}}$ The same year, Wilson and Webb reported the first chemically responsive channel in which palladium ions were used to generate cation channels by complexing pyridyl-cholate lipids into membrane-spanning compounds. ${ }^{\mathbf{4 7 1 , 4 7 2}}$ The addition of a competing ligand such as hexathia-18-crown-6 induced the closure of the channel, whereas outer decoration of the cholates with biotin moieties permits its complexation with avidin to control the ion flow across the membrane. The group of Matile has also demonstrated the possible multifunctionality of a single self-assembled system. ${ }^{473}$ Indeed, $p$-octiphenyl rods decorated with naphthalene units were shown to self-assemble into a quadruple $\pi$ - $M$-helix that displays photosynthetic activity when inserted in vesicles with a well-defined environment. Addition of electron-rich ligands causes the opening of the helix into a barrel-stave ion channel selective for anions. ${ }^{474}$ This group has also taken advantage of various supramolecular interactions to develop innovative nanopores. ${ }^{475}$ In collaboration with Guichard and coworkers, they showed that oligourea/amide macrocycles can be inserted in bilayer membranes and serve as selective anion transporters. ${ }^{\mathbf{4 7}}$ In that case, anion-macrodipole and macrodipole-potential interactions were shown to be responsible for anion selectivity and voltage sensitivity, respectively. In another example, by using a collection of naphthalenediimide (NDI), this group also demonstrated the relevance of anion- $\pi$ interactions for the transport of anions in bilayer membranes. ${ }^{477,478}$ In particular, they have shown that $\pi$-acidification and decrowding of the active site, namely NDI, play an important role in the selective transport of chlorine over bromine and iodine ions. ${ }^{47}$ Conversely, high supramolecular organization inducing $\pi, \pi$-enhanced anion- $\pi$ interactions was shown to account for the preferential transport of nitrate over acetate and a poorer selectivity towards halogen ions. Recently, Matile and coworkers have exploited the hydrophobicity, directionality, and strength of halogen bonds in small perfluorinated molecules, even the highly volatile trifluoroiodomethane, to transport anions across lipid bilayers. ${ }^{479}$ The absence of leakage and the observed cooperativity and selectivity make this small and simple system particularly attractive for applications in life and material sciences. Finally, it should be noted that artificial $\beta$-barrels developed by this group and mentioned in Section 4.1 have not only been used as sensors but also as transport systems across lipid membranes. ${ }^{403,463,464}$

\subsubsection{Nanomaterials for purification and separation} purposes

Nanostructured membranes. One important tool in environmental science is the use of membranes to remove any contaminants that might be present in water. ${ }^{480,481}$ In 2007 , Moore and coworkers used six-arm rigid star amphiphiles to produce nanofiltration membranes over a block copolymeric film that rival the best commercially available membranes for the rejection of Rhodamine Wt and As(III) together with a two- to three-fold higher water flux. ${ }^{\mathbf{8 2}}$ Two years later, the group of Ichinose reported the formation of ferritin-based membranes of 30-100 nm thickness that were not only stable in organic solvents and for a wide range of $\mathrm{pH}$ (1.5-13) but also mechanically robust. ${ }^{483}$ This property was confirmed by the ability of the system to withstand water fluxes that are more than 1000 times the ones supported by commercially available membranes while displaying similar filtration properties. Recently, Rybtchinski and coworkers demonstrated the recyclability of a supramolecular membrane made of perylene-based amphiphilic molecules and its capacity to separate nanoparticles by size (Fig. 12a). ${ }^{\mathbf{4 8 0 , 4 8 4}}$ The supramolecular membrane with an easily controlled thickness is formed after filtration over a commercial microfiltration syringe filter of a water solution of bis-perylene displayed in Fig. 12a(v). Filtration of a water solution of nanoparticles allows the direct collection of sub-5 $\mathrm{nm}$ nanoparticles in the filtrate and retention of the larger ones on the filter (Fig. 12a(iv)). Washing of the filter with a water-ethanol mixture disrupts the supramolecular membrane and allows the subsequent recovery of the large nanoparticles. Interestingly, this membrane could be reused up to four times without losing its properties and was also applied to the size-exclusion chromatography of sub-5 nm quantum dots with a reasonable selectivity. In 2012, the groups of Barboiu and Quemener have shown that $50 \mathrm{~nm}$ diameter micelles made of triblock copolymers can form nanoporous supramolecular films with tunable porosity depending on the water pressure applied. ${ }^{485}$ This adaptive behavior under an external stimulus (pressure) was confirmed by the self-healing ability of the membrane under increasing water flow pressures. The group of Barbiou has recently developed a number of adaptive membranes for the transport of gas or ions using dynamic constitutional systems and 

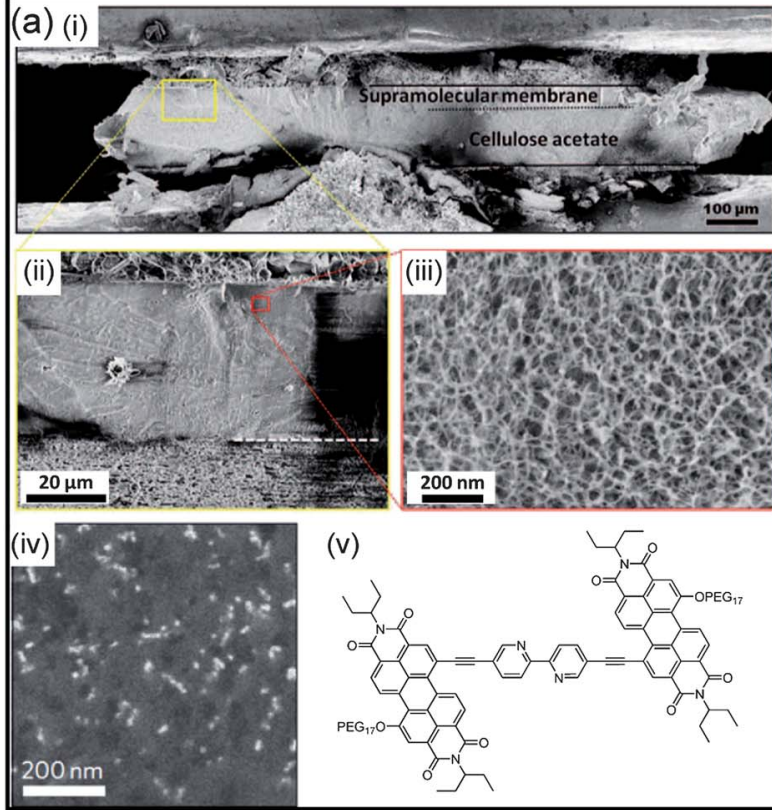

(v)
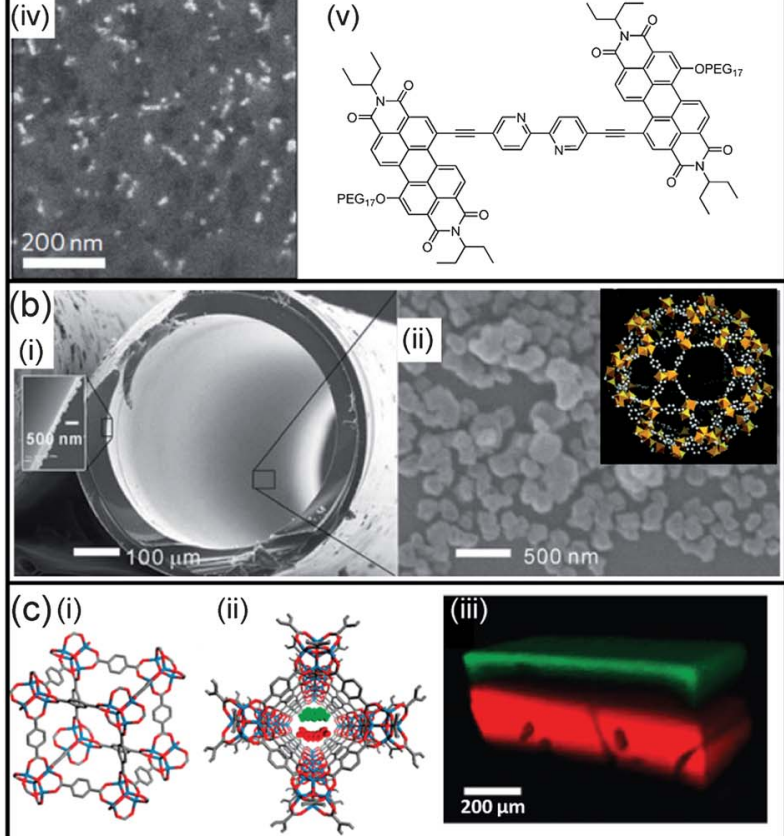

(ii)
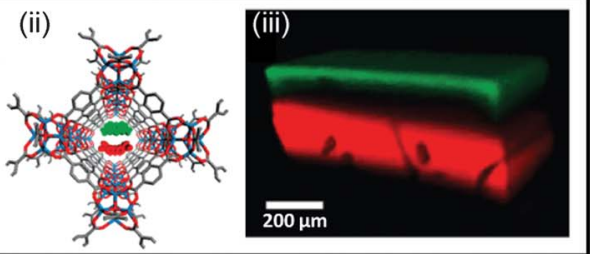

Fig. 12 Self-assembled nanomaterials for separation and transport. (a) (i) CryoSEM image of the membrane's cross-section; (ii) magnified area showing the sharp border between the supramolecular membrane and its cellulose acetate support; (iii) high magnification reveals the porous three-dimensional nanostructure of the supramolecular membrane material; (iv) backscattered electron cryo-SEM image of the supramolecular membrane used for filtering a solution of gold nanoparticles. Gold nanoparticles (appearing as bright spots) have sizes ranging from 10 to $20 \mathrm{~nm}$; (v) amphiphilic perylene used as a self-assembly unit for the supramolecular membrane. (b) SEM images of (i) the cross-section of MIL-101 coated capillary column (inset shows the thickness of the MIL-101 coating); and (ii) MIL101 deposited on the inner wall of the capillary column (inset shows the structure of MIL-101). (c) (i) One unit cell of a MOF-5 crystal made of terephthalic acid struts and octahedral carboxylato $\mathrm{Zn}_{4} \mathrm{O}\left(\mathrm{CO}_{2}\right)_{6}$ secondary building units; (ii) view along the 1D channel of MOF-5. The cross-section of the channel is $\sim 8 \times 8 \AA^{2}$. The red and green molecules in the channel illustrate the mixture to be separated; (iii) 3D fluorescence confocal images of MOF-5 crystals in which the migrating dyes are resolved into separate bands: separation of pyronin B (red) and thionin (green) at 90 min. Panel (a): reproduced with permission from ref. 484; (b) reproduced with permission from ref. 496; (c) reproduced with permission from ref. 500.

supramolecular chemistry. ${ }^{\mathbf{4 8 1 , 4 8 6 - 4 9 0}}$ For example, they have reported one of the rare examples of a water and proton transporter $^{491}$ using ureidoimidazole quartets that form barreled channels in a membrane environment, the system being inert for transporting $\mathrm{Li}^{+}$and $\mathrm{Na}^{+}{ }^{487}$
MOFs. MOFs have been used to selectively store gases or small molecules (see Section 4.2), so that their use in separating devices has become evident. ${ }^{\mathbf{4 9 2 , 4 9 3}}$ More than five years ago, the group of De Vos reported the successful separation in the liquid phase of mixed $\mathrm{C}_{8}$ alkylaromatic compounds using MIL-47 as the absorbent, due to an efficient packing of the various isomers in the pores. ${ }^{494}$ The difference of retention observed for the xylene isomers comes from the relative alignment of their aromatic planes within a pair located in one pore. The same MOF could also be used to separate ethylbenzene from styrene with baseline-separated peaks. ${ }^{495}$ A similar separation was achieved using MIL-53 at room temperature, but the flexibility of the framework induced a loss of resolution at higher temperatures due to enthalpic considerations. Nevertheless, MIL-53 might be relevant for industrial application as it is capable of removing typical impurities ( $o$-xylene, toluene) originating from the synthetic process. Considering the importance of aromatic molecules in industrial processes but also regarding environmental concerns, $\mathrm{Gu}$ and Yan reported the fabrication of a capillary column coated with MIL-101 and its application in gas-phase chromatography for the separation of the xylene isomers (Fig. 12b). ${ }^{496}$

Not only host-guest interactions, but also the presence of unsaturated coordination sites and the polarity of the MOF were shown to account for the fast and excellent separation of benzene derivatives or $n$-alkane mixtures. Extension of this work using other MOFs and/or surface-coating techniques for the separation of environmentally relevant samples, such as natural gas or petrol, clearly establishes MOFs as promising coating phases for high resolution gas chromatography. ${ }^{497,498}$ The groups of Bryliakov and Fedin demonstrated also the potential of a chiral MOF to efficiently separate sulfoxide enantiomers with an enantiomeric excess up to $60 \%{ }^{499}$ They have even shown the possibility of performing a one-pot oxidation/purification process on thioethers, pointing out the dual role played by the MOF as both the catalyst and stationary phase. Millimeter-sized MOF single crystals presenting nanometric pores have also been used as "chromatographic columns" to separate aromatic dyes (Fig. 12c). ${ }^{500}$ In this case, the sizes of the molecules along with their supramolecular interactions with the pores account for the selectivities observed during chromatography. Selective gas membranes have also been elaborated using MOFs either as thin films or as additives to mixed-matrix membranes. ${ }^{\mathbf{4 9 2}}$ For example, Caro and coworkers took advantage of the difference in kinetic parameters between gases $\left(\mathrm{H}_{2}, \mathrm{CO}_{2}, \mathrm{~N}_{2}, \mathrm{CH}_{4}\right)$ to produce a membrane made of a ZIF-7 thin film over an alumina support that enabled the selective filtration of hydrogen over the other gases with a very good thermal stability. ${ }^{501} \mathrm{~A}$ similar molecular sieving effect was also observed for the separation of small gas molecules $\left(\mathrm{H}_{2}, \mathrm{CO}_{2}\right)$ from larger ones such as methane or propane using a polymeric membrane coated with ZIF-8 due to their ability to fit into the pores of the MOF ${ }^{502}$ For environmental applications, MOF-based materials are not far from being used in practical applications, although issues regarding their stability, scale-up and the design of the separation process remain to be addressed. ${ }^{492}$ 
Host-guest devices. As mentioned previously, this review is not dealing with simple host-guest recognition events. However, regarding separation and purification purposes, many devices taking advantages of this phenomenon have been reported and could be practically used for challenging separations. ${ }^{\mathbf{5 0 3 , 5 0 4}}$ One particular example is the purification of singlewalled carbon nanotubes (SWNTs), whose chiralities highly impact their optical, electrical, and mechanical properties. ${ }^{505}$ For more than five years, chemists have taken advantage of supramolecular interactions between SWNTs and chiral diporphyrin or DNA, for instance, to produce optically active nanotubes. ${ }^{\mathbf{5 0 6 , 5 0 7}}$ Macrocylic hosts inducing supramolecular interactions with the guest have also been used for the separation of enantiomers such as fullerene $\mathrm{C}_{76}$ or various organic compounds, and could potentially be used as chiral stationary phases in HPLC columns. ${ }^{\mathbf{5 0 8 , 5 0 9}}$ Finally, the reversible affinity between boronic acids and diols has been used to produce modified gel electrophoresis that can separate unambiguously saccharides and glycated from unglycated proteins, separations that cannot be achieved using classical electrophoretic techniques. ${ }^{\mathbf{5 1 0 , 5 1 1}}$

\subsection{Nanomaterials for catalysis}

Reducing the costs and wastes of chemical reactions is one of the major environmental issues for industry and catalysts are very important tools in that direction. In living organisms, enzymes perform efficient catalytic reactions in confined and well-organized environments. Chemists have always been inspired by this structure/property relationship and they still actively try to develop small complexes or large supramolecular assemblies that can compete in these ranges of efficiency and selectivity, but also in the ability to regulate their activity using allosteric effects. ${ }^{512-514}$

Capsules and supramolecular cages. For more than 30 years, chemists have tried to mimic the confined space used by enzymes to produce chemical reactions by synthesizing single container molecules (which will not be discussed in this review) or by taking advantage of self-organization to produce supramolecular cages of nanometric size..$^{515-517}$ Recently, this latter approach was not only used to accelerate the reaction but also to promote unusual selectivities and very efficient catalytic turnovers. In 2006, Fujita and coworkers reported a palladium cage which promotes a Diels-Alder reaction between anthracene and maleimide derivatives in water with an interesting regioselectivity, due to the spatial restriction imposed by the host. ${ }^{518}$ The same cage was then used catalytically to promote the Knoevenagel condensation of various aromatic aldehydes in water, due to the enhanced stabilization of the anionic intermediate in the cationic environment of the cage and the fast release of the product as a result of its too large size compared with the host. ${ }^{519}$ In the absence of the host, the same reactions were shown to barely proceed. This stabilization of a reaction intermediate in a host by using electrostatic interactions was also applied by the groups of Bergman and Raymond for the catalytic hydrolysis of various orthoformates in a basic solution whereas these chemicals are known to be highly stable in such a medium and with a highly enhanced reaction rate compared to the uncatalyzed reaction. ${ }^{\mathbf{5 2 0}}$ This supramolecular cage was also shown to be very efficient in the Nazarov cyclisation of 1,3pentanedienols, the cyclization of terpenes, the cycloisomerisation of enynes, or even in a tandem enzymatic kinetic resolution/cyclization of allenes. ${ }^{\mathbf{5 2 1 - 5 2 3}}$

Nanopores. In general, any supramolecular structure presenting a confined space that can accommodate small molecules could be suitable for catalysis. In the catalytic site of enzymes, the additional internal order and the presence of polarizing groups such as histidine residues are known to enhance their activity and selectivity. Molecules with selfassembling properties and presenting histidine residues or imidazole moieties have been developed in order to mimic these natural systems. In 2003, the group of Matile reported the first example of a catalytic pore made of $p$-octiphenyl rods bearing $\beta$-sheets-forming peptides with arginine/histidine dyads inserted in large unilamellar vesicles for the esterolysis of 8-acetoxypyrene-1,3,6-trisulfonate. ${ }^{\mathbf{2 2 4 , 5 2 5}}$ Interestingly, if polarized membranes were used, an increase in the $V_{\max }$ of the trans esterolysis was observed, corresponding to an acceleration of substrate/product binding/release by constructive electrostatic steering.

Nanofibers. Similarly, Guler and Stupp have used peptide amphiphiles bearing histidine residues to produce self-assembled nanofibers with a high aspect-ratio and hydrolytic properties regarding 2,4-dinitrophenyl acetate. ${ }^{\mathbf{5 2 6}}$ Interestingly, when the residues of the peptide amphiphiles were changed to induce the formation of spherical aggregates, the hydrolytic property was lost, demonstrating the importance of the internal ordering of the nanostructure for the catalytic function.

Nanoparticles. Another approach that has taken advantage of the histidine residues for the hydrolysis of esters consists in using peptide-nanoparticle complexes formed by electrostatic interactions between a cationic gold nanoparticle and an anionic peptide, which do not display by themselves any catalytic activity. ${ }^{527}$ The role of the cationic gold nanoparticles is twofold: (i) bringing together the catalyst and the substrate in close proximity, which results in an increase of the effective molarity, and (ii) generating a local $\mathrm{pH}$ increase that enhances the catalytic properties of the histidine residues.

Porous crystals. In Section 4.2, we have mentioned the importance of porous crystals for chemical storage. In particular, Shimizu and coworkers have shown that bis-urea macrocycles can accommodate various guests ranging from carbon dioxide or acetic acid to xylene derivatives. ${ }^{425}$ In 2008 , the same self-assembled porous crystals were used to promote the $[2+2]$ cycloaddition of various $\alpha, \beta$-unsaturated ketones. ${ }^{528}$ As confirmed by molecular modeling, the perfect match between the size/shape of the guest and the dimensions of the internal cavity of the host accounts for the enhanced conversion and exo head-to-tail selectivity observed in this templated reaction. Recently, the same group reported the formation of a porous crystal made of a larger bis-urea macrocycle that can accommodate coumarin and facilitate its selective photodimerization. ${ }^{529}$ Indeed, an unusual and very high selectivity towards the anti head-to-head dimer was observed, probably 
due to a stabilization of the coumarin within the host via $\pi-\pi$ interactions. Nanoporous organic crystals made of dipeptides incorporating a valine and either an alanine or an isoleucine derivative ${ }^{530}$ have also been recently used for the gamma-rayinduced polymerization of acrylonitrile, pentadiene, and isoprene (Fig. 13a). ${ }^{531}$ Only the 1,4-trans polymers with high molecular weights were obtained from diene monomers as the geometry of the pores forbids all other configurations. For acrylonitrile, the isotactic polymer was exclusively obtained under mild temperature and pressure, a performance that cannot be achieved using classical polymerization techniques. Post-modifications of the obtained polymers could also be carried out in the nanochannels. Removal of the peptidic matrix was also easily achieved without disrupting the rigidity of the polymeric scaffold.

Supramolecular gels. Small molecules that display sol-gel transitions have also been reported as efficient catalysts in the gel state. ${ }^{532}$ For example, the group of Escuder and Miravet has reported the enhanced yield of the Henry nitroaldol reaction between 4-nitrobenzaldehyde and nitromethane when the reaction is performed in the gel state. ${ }^{533}$ Interestingly, as the sol-gel transition occurs at a given temperature, the catalysis can be easily controlled by varying this stimulus. Another approach for the catalysis in the gel state is the formation of self-supported metallogels incorporating metal ions that both promote the formation of the gel and serve as catalytic species. For instance, the group of Zhang reported the formation of xerogels made of tripodal pyridinyl ligands and various amounts of palladium ions that are very efficient for SuzukiMiyaura cross-couplings (Fig. 13d). ${ }^{534}$ Depending on the palladium-ligand ratio, gels with various morphologies, ranging from spherical or worm-like particles up to fibrous networks, were obtained and all display efficient catalytic properties with kinetics that depend on this ratio. Interestingly, for a same metal/ligand ratio, the xerogel presents a much better recyclability than the gel with no loss of activity after 5 reuses.

MOFs. As demonstrated in the previous sections, MOFs have attracted a lot of attention in the field of environmental sciences. $^{340,341}$ Their intrinsic porosity along with their stability in organic solvents has promoted them as suitable tools for heterogeneous catalysis..$^{535-538}$ In particular, chiral MOFs have been actively developed to perform asymmetric catalysis with efficiencies that rival the one observed in homogeneous systems. Two strategies can be followed to produce chiral MOFs: the use of chiral building blocks to form the structure or the post-modification of an achiral framework with chiral units. For example, MOFs made of metal/salen building blocks have been successfully applied to the cyclopropanation of various substituted olefins with diastereomeric and enantiomeric excesses (e.e.) that compete with homogeneous catalysts. ${ }^{539}$ They have also been very efficient in the hydrolytic kinetic resolution of epoxides with very high e.e. and at lower substrate/ catalyst ratios than in homogeneous medium. ${ }^{540}$ Chiral MOFs have also been successfully applied to organocatalysis. For example, the asymmetric $\alpha$-alkylation of aliphatic aldehydes was achieved using tandem photo- and organocatalysis with a single MOF incorporating both active units. ${ }^{541}$ Post- modification of an achiral framework with proline derivatives allowed the aldol reaction with higher yield and e.e. than the corresponding homogeneous catalyst. ${ }^{542}$ Finally, MOFs made of chiral building blocks can also be further functionalized with metal ions. This strategy was applied to the catalytic transformation of aromatic aldehydes in chiral secondary alcohols using alkynylzinc reagents with very high conversion and enantiomeric excess, although this value was shown to be dependent on the size of the open channel. ${ }^{543}$ It should be mentioned that MOFs have also been used as porous matrices for metal nanoparticles and successfully applied in catalysis with high catalytic efficiencies. ${ }^{544}$

Nanotubes. One particular example of MOFs concerns 3D metal-organic nanotubes that display 1D nanotubular channels. ${ }^{545}$ The large width of the channels $(3.3 \mathrm{~nm})$ allows an easy post-modification with palladium ions of the $N$-heterocyclic carbenes embedded in the nanotubes that still display a very efficient substrate uptake. This MOF presents a very high efficiency at low loading in heterogeneous Suzuki-Miyaura and Heck cross-coupling reactions or hydrogenation of olefins, whereas the individual components barely promote the same reactions. Similarly, the group of Zhou has reported the formation of a heme-like MOF displaying nanometer-sized tubular channels that promotes the very efficient oxidation of various substrates following a biomimetic process. ${ }^{546}$

Polymersomes. Block copolymers are known to produce various kinds of morphologies ranging from micelles or vesicles to lamellae. ${ }^{547}$ In particular, amphiphilic block copolymers are known to form polymersomes that are analogous to liposomes but with different properties associated with their larger size. In 2007, the groups of Rowan and van Hest reported the use of such structures to encapsulate enzymes for producing a nanoreactor capable of performing one-pot multistep reactions. ${ }^{548}$ Several years later, the same group demonstrated that crosslinked polymersomes can serve as colloidal nanoparticles to stabilize a Pickering emulsion that incorporates enzymes either in the water phase (Fig. 13b(iii)) or in the lumen of the polymersome (Fig. 13b(iv)). ${ }^{549}$ In both cases, the catalytic activity for esterification or hydrolysis was superior to the one observed using the same enzyme in a biphasic system. Additionally, the Pickering emulsions were easily recycled and reused up to 8 times while maintaining efficient activity.

Micelles and vesicles. Micelles and vesicles are attractive objects for performing catalysis as they tend to mimic liposomes and cells. We have recently reviewed reactions taking place in dynamic self-assemblies, particularly when such assemblies are made of surfactants, block copolymers or amphiphilic peptides. ${ }^{550}$ Therefore, only few examples from late 2011 and 2012 will be highlighted. For example, the group of Lipschutz has shown that vitamin E-derived amphiphiles can be used to promote gold-catalyzed cycloisomerization or coppercatalyzed 1,4-additions in a water medium. ${ }^{551,552}$ In both cases, very high yields were obtained on a large variety of substrates and the micellar catalytic system could be easily recycled with no loss of activity. Deng and coworkers have also shown that a novel chiral surfactant-type catalyst that directly incorporates the metal ions can form nanometric micelles which display very 


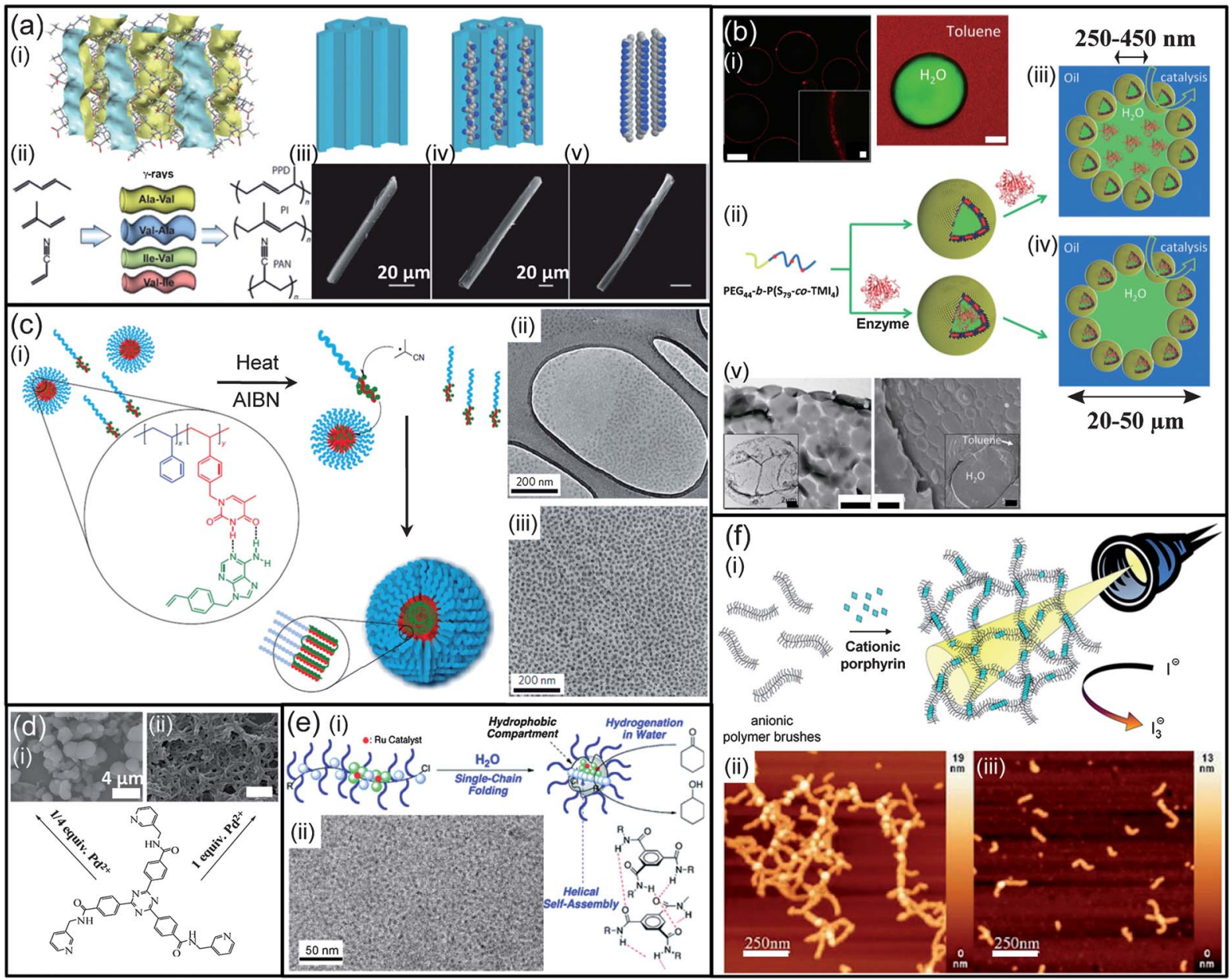

Fig. 13 Self-assembled nanomaterials for catalysis. (a) (i) Crystal structure of the porous Ala-Val compound showing the empty channels along the $c$ axis in blue and yellow; (ii) schematic representation of the monomers and dipeptides used for the polymerization process; SEM images of (iii) an individual Ala-Val crystal, (iv) Ala-Val/ PAN crystalline adduct, (v) ladder-polymer fibril after removal of the crystal; a schematic representation of the process at the molecular level is shown above, PAN: poly(acrylonitrile). (b) (i) CLSM images of polymersome Pickering emulsions (water in toluene); left: polymersomes are stained by rhodamine B (scale bar: $20 \mu \mathrm{m}$ ); the inset is a magnified image (scale bar: $2 \mu \mathrm{m})$; right: FITC-Dex $(4.4 \mathrm{kDa}$ ) and Nile red were dissolved in water and toluene, respectively (scale bar: $10 \mu \mathrm{m})$; (ii) representation of the crosslinking process to prepare stabilized polymersomes and schematic representation of a Pickering emulsion with the enzyme in the water phase (iii), or with the enzyme inside the polymersome lumen (iv); (v) TEM image (scale bar: $500 \mathrm{~nm}$ ) and cryo-SEM image (scale bar: 500 nm) of polymersome Pickering emulsions; the insets are overviews at lower magnification (left inset: scale bar $2 \mu \mathrm{m}$, right inset: scale bar $5 \mu \mathrm{m}$ ). (c) (i) On addition of AlBN and heating, it is proposed that vinylbenzyl adenine (VBA) initiation occurs on an exchanging unimer before returning to a micelle made of the block copolymer PSt- $b$-PVBT (poly(styrene-b-vinylbenzyl thymine)) for continued propagation. Further dynamic VBA-loaded unimers add to the initiated micelle, from a reservoir of non-initiated micelles, to yield a stable, non-dynamic

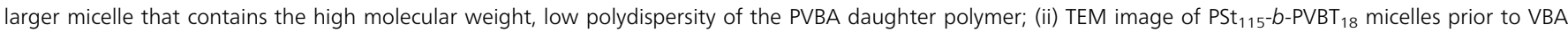
addition, the lighter region is the grapheme oxide coating over a hole in the lacey carbon support (darker/thicker region); (iii) TEM image of micelles obtained after the addition and polymerization of VBA in the presence of PSt ${ }_{115}-$ b-PVBT 18 . (d) SEM images of xerogels made of a large semirigid tripodal ligand, 4,4',4"-(1,3,5-triazine2,4,6-triyl)-tris( $N$-(pyridin-3-ylmethyl)benzamide) in different $\mathrm{Pd} / \mathrm{L}$ ratios of (i) $1: 4$ and (ii) $1: 1$. (e) (i) Supramolecular single-chain folding of polymers in water affording a compartmentalized catalyst for the transfer hydrogenation of ketones; (ii) cryo-TEM images of the Ru-PEGMA/BTAMA/SDP segmented terpolymer ( $M_{w}$, SLS $=105000, R_{\mathrm{h}}, \mathrm{DLS}=6.8 \mathrm{~nm}$ ): Magnification $=62 \mathrm{k}$, PEGMA: poly(ethylene glycol) methyl ether methacrylate, BTAMA: benzene-1,3,5-tricarboxamide-bearing methacrylate, SDP: diphenylphosphinostyrene. (f) (i) Schematic representation of supramolecular structure formation between cylindrical polystyrene sulfonate (PSS) brushes and cationic tetravalent porphyrins (TMPyP) and their use as a photocatalyst; atomic force microscopy (AFM) of a TMPyP/PSS-brush sample with a charge ratio of I : 0.4 deposited onto a mica surface: (ii) sample prepared in aqueous solution, (iii) sample prepared in aqueous solution with $c\left(\right.$ iodide) $=0.01$ mol $\mathrm{L}^{-1}$. Panel (a): adapted with permission from ref. 531; (b) reproduced with permission from ref. 549; (c) adapted with permission from ref. 554; (d) reproduced with permission from ref. 534; (e) adapted with permission from ref. 556; (f) reproduced with permission from ref. 560.

high efficiencies in terms of yield and e.e. for the asymmetric transfer hydrogenation of various aliphatic ketones. ${ }^{553}$ TEM images confirmed that the reaction is taking place in the micelle as their size increases three-fold in the reaction mixture. Finally, the group of O'Reilly reported recently that the core of a block copolymer micelle can serve as a template for controlled radical polymerization (Fig. 13c). ${ }^{554}$ Unexpected high molecular weight polymers with low polydispersity were obtained and TEM imaging clearly demonstrates that the polymerization takes place in the core of the micelle as its size changes from $\sim 13 \mathrm{~nm}$ before addition of both the monomer and the initiator to $\sim 20 \mathrm{~nm}$ after polymerization (Fig. 13c(ii) and (iii)). 
Folding polymers. The folding ability of covalent polymers has also been recently exploited as well-defined compartments can be created which serve as the catalytic unit. In 2009, Gellman, Hilvert, and coworkers reported the catalytic properties of a decameric peptidic foldamer in retroaldol reactions. ${ }^{555}$ Both the helical folding and the formation of large assemblies due to hydrophobic interactions were shown to account for the excellent catalytic efficiency of the system. Recently, the group of Meijer and Palmans reported the synthesis of terpolymers incorporating chiral benzene-1,3,5-tricarboxamide units that fold with a preferential handedness in suitable solvents (Fig. 13e).$^{556-558}$ In water, terpolymers including either ruthenium catalysts or proline derivatives collapse into single-chain nanoparticles with a well-structured inner compartment suitable for catalysis. Both hydrogenation of ketones and aldol reactions proceeded with excellent conversion at low catalyst loading.

Polymer networks. Finally, supramolecular interactions such as hydrogen bonding or electrostatic interactions can be used to generate polymer networks which display catalytic properties not achieved by the single polymer. In 2009, Willner and coworkers took advantage of the self-assembling properties of DNA to form hexagon-like DNA strips which were then further non-covalently modified by two enzymes or a cofactor-enzyme pair. ${ }^{559}$ In both cases, biocatalysis was observed whereas no activity was detected in the absence of DNA templates. Recently, Frühbeißer and Gröhn have shown that anionic polymer brushes can be non-covalently reticulated using porphyrin derivatives to form polymer networks that display photocatalytic properties (Fig. 13f). ${ }^{560}$ The efficiency of the porphyrin in the light-induced oxidation of iodine was more than 8 times higher when embedded in the polymer network than without a template. This result is probably due to the increase in effective molarity within a preorganized structure, which is one of the main reasons why self-assembled structures have been particularly attractive for catalysis.

\section{Conclusion}

The bottom-up construction of nanostructures based on supramolecular self-assembly has been a subject of utmost fundamental interest for the past forty years and chemists have acquired a high control of objects at that scale by encoding their molecular constituents. We are currently witnessing that this field is sufficiently mature to apply its construction rules towards functional nanomaterials with novel properties related to their sizes, defined structures, and dynamics. This review illustrates how such materials afford a series of interesting responses to crucial societal problems for health, energy, and environment. Beyond this snapshot of recent advances, one can easily imagine that such a blossoming technology has still plenty of room to expand and will certainly produce even more sophisticated nanomaterials in the future. In particular, many challenges remain in the fundamental understanding of selforganization at the nanoscale when coming to the study of far from equilibrium self-assembled systems and networks. We believe, among others, that the expected progress in this challenging field related to complexity and emergence will dramatically affect the design and potential of the next generation of nanomaterials with the ultimate goal to bridge the gap, one day, between materials and life science.

\section{Acknowledgements}

We thank the European Research Council under the European Community's Seventh Framework Program (FP7/2007-2013)/ ERC Starting Grant agreement no. 257099. We also thank the Agence Nationale pour la Recherche (ANR-09-BLAN-034-02) and a Marie Curie Career Integration Grant for postdoctoral fellowships (E. B. and Y. R.). We acknowledge the University of Strasbourg (UdS), the Centre National de la Recherche Scientifique (CNRS), the international center for Frontier Research in Chemistry (icFRC), the Laboratory of Excellence for Complex Systems Chemistry (LabEx CSC), and the Institut Universitaire de France (IUF).

\section{Notes and references}

1 G. M. Whitesides and B. Grzybowski, Science, 2002, 295, 2418-2421.

2 J.-M. Lehn, Supramolecular Chemistry, WILEY-VCH Verlag GmbH, Weinheim, 1995.

3 Supramolecular Chemistry: From Molecules to Nanomaterials, ed. P. Gale and J. Steed, Wiley-VCH Verlag GmbH \& Co. KGaA, Weinheim, 2012.

4 Nanomedicine, in Nanotechnology, ed. H. Fuchs, M. Grätzel, H. Krug, G. Schmid, V. Vogel and R. Waser, Wiley-VCH Verlag GmbH \& Co. KGaA, Weinheim, 2010, vol. 5.

5 Nanomaterials for Medical Applications, ed. Z. P. Aguilar, Elsevier, Amsterdam, 2013.

6 NanoBiotechnology, ed. C. M. Niemeyer and C. A. Mirkin, Wiley-VCH Verlag GmbH \& Co. KGaA, Weinheim, 2004.

7 Nanobiotechnology II, ed. C. M. Niemeyer and C. A. Mirkin, Wiley-VCH Verlag GmbH \& Co. KGaA, Weinheim, 2007.

8 Z. P. Aguilar, Chapter 4-Nanobiosensors, in Nanomaterials for Medical Applications, ed. A. Zoraida, Elsevier, 2013, pp. 127179.

9 Nanomaterials for Medical Diagnosis and Therapy, ed. C. S. S. R. Kumar, Wiley-VCH Verlag GmbH \& Co. KGaA, Weinheim, 2007.

10 Nanomaterials in Drug Delivery, Imaging, and Tissue Engineering, ed. A. Tiwari and A. Tiwari, Wiley-VCH Verlag GmbH \& Co. KGaA, Weinheim, 2013.

11 J. Lei and H. Ju, Chem. Soc. Rev., 2012, 41, 2122-2134.

12 K. B. Cederquist and S. O. Kelley, Curr. Opin. Chem. Biol., 2012, 16, 415-421.

13 H. Jans and Q. Huo, Chem. Soc. Rev., 2012, 41, 2849-2866. 14 N. L. Rosi and C. A. Mirkin, Chem. Rev., 2005, 105, 15471562.

15 M. E. Stewart, C. R. Anderton, L. B. Thompson, J. Maria, S. K. Gray, J. A. Rogers and R. G. Nuzzo, Chem. Rev., 2008, 108, 494-521.

16 T. L. Doane and C. Burda, Chem. Soc. Rev., 2012, 41, 28852911. 
17 S. K. Ghosh and T. Pal, Chem. Rev., 2007, 107, 4797-4862.

18 R. Elghanian, J. J. Storhoff, R. C. Mucic, R. L. Letsinger and C. A. Mirkin, Science, 1997, 277, 1078-1081.

19 S. Y. Park, A. K. R. Lytton-Jean, B. Lee, S. Weigand, G. C. Schatz and C. A. Mirkin, Nature, 2008, 451, 553556.

20 J.-M. Nam, C. S. Thaxton and C. A. Mirkin, Science, 2003, 301, 1884-1886.

21 D. G. Georganopoulou, L. Chang, J.-M. Nam, C. S. Thaxton, E. J. Mufson, W. L. Klein and C. A. Mirkin, Proc. Natl. Acad. Sci. U. S. A., 2005, 102, 2273-2276.

22 R. de la Rica, D. Aili and M. M. Stevens, Adv. Drug Delivery Rev., 2012, 64, 967-978.

23 A. Laromaine, L. Koh, M. Murugesan, R. V. Ulijn and M. M. Stevens, J. Am. Chem. Soc., 2007, 129, 4156-4157.

24 D. Aili, R. Selegård, L. Baltzer, K. Enander and B. Liedberg, Small, 2009, 5, 2445-2452.

25 S. Gupta, H. Andresen, J. E. Ghadiali and M. M. Stevens, Small, 2010, 6, 1509-1513.

26 D. Aili, M. Mager, D. Roche and M. M. Stevens, Nano Lett., 2010, 11, 1401-1405.

27 X. Liu, Q. Dai, L. Austin, J. Coutts, G. Knowles, J. Zou, H. Chen and Q. Huo, J. Am. Chem. Soc., 2008, 130, 27802782.

28 C.-C. You, O. R. Miranda, B. Gider, P. S. Ghosh, I.-B. Kim, B. Erdogan, S. A. Krovi, U. H. F. Bunz and V. M. Rotello, Nat. Nanotechnol., 2007, 2, 318-323.

29 O. R. Miranda, B. Creran and V. M. Rotello, Curr. Opin. Chem. Biol., 2010, 14, 728-736.

$30 \mathrm{X} . \mathrm{Xu}, \mathrm{B}$. Lin, J. Feng, Y. Wang, S. Cheng, X. Zhang and R. Zhuo, Macromol. Rapid Commun., 2012, 33, 426-431.

31 G. Liang, H. Ren and J. Rao, Nat. Chem., 2010, 2, 54-60.

32 C.-Y. Cao, Y. Chen, F.-Z. Wu, Y. Deng and G.-L. Liang, Chem. Commun., 2011, 47, 10320-10322.

33 C. J. Musto and K. S. Suslick, Curr. Opin. Chem. Biol., 2010, 14, 758-766.

34 N. Y. Edwards, T. W. Sager, J. T. McDevitt and E. V. Anslyn, J. Am. Chem. Soc., 2007, 129, 13575-13583.

35 H. Özhalici-Ünal and B. A. Armitage, ACS Nano, 2009, 3, 425-433.

36 C. Lin, R. Jungmann, A. M. Leifer, C. Li, D. Levner, G. M. Church, W. M. Shih and P. Yin, Nat. Chem., 2012, 4, 832-839.

37 E. M. Cornett, E. A. Campbell, G. Gulenay, E. Peterson, N. Bhaskar and D. M. Kolpashchikov, Angew. Chem., Int. Ed., 2012, 51, 9075-9077.

38 I. Koh, R. Hong, R. Weissleder and L. Josephson, Angew. Chem., Int. Ed., 2008, 47, 4119-4121.

39 H. Lee, E. Sun, D. Ham and R. Weissleder, Nat. Med., 2008, 14, 869-874.

40 P. Besenius, G. Portale, P. H. H. Bomans, H. M. Janssen, A. R. A. Palmans and E. W. Meijer, Proc. Natl. Acad. Sci. U. S. A., 2010, 107, 17888-17893.

41 P. Besenius, J. L. M. Heynens, R. Straathof, M. M. L. Nieuwenhuizen, P. H. H. Bomans, E. Terreno, S. Aime, G. J. Strijkers, K. Nicolay and E. W. Meijer, Contrast Media Mol. Imaging, 2012, 7, 356-361.
42 C.-Y. Cao, Y.-Y. Shen, J.-D. Wang, L. Li and G.-L. Liang, Sci. Rep., 2013, 3, 1024.

43 S.-J. Park, T. A. Taton and C. A. Mirkin, Science, 2002, 295, 1503-1506.

44 B. He, T. J. Morrow and C. D. Keating, Curr. Opin. Chem. Biol., 2008, 12, 522-528.

45 C. M. Lieber, MRS Bull., 2011, 36, 1052-1063.

46 H. Lord and S. O. Kelley, J. Mater. Chem., 2009, 19, 31273134.

47 J.-I. Hahm and C. M. Lieber, Nano Lett., 2003, 4, 51-54.

48 Y. L. Bunimovich, Y. S. Shin, W.-S. Yeo, M. Amori, G. Kwong and J. R. Heath, J. Am. Chem. Soc., 2006, 128, 16323-16331.

49 A. Star, E. Tu, J. Niemann, J.-C. P. Gabriel, C. S. Joiner and C. Valcke, Proc. Natl. Acad. Sci. U. S. A., 2006, 103, 921-926.

50 S. N. Kim, J. F. Rusling and F. Papadimitrakopoulos, $A d v$. Mater., 2007, 19, 3214-3228.

51 Z. Wu, Z. Zhen, J.-H. Jiang, G.-L. Shen and R.-Q. Yu, J. Am. Chem. Soc., 2009, 131, 12325-12332.

52 L. Adler-Abramovich, M. Badihi-Mossberg, E. Gazit and J. Rishpon, Small, 2010, 6, 825-831.

53 L. Sasso, I. Vedarethinam, J. Emnéus, W. E. Svendsen and J. Castillo-León, J. Nanosci. Nanotechnol., 2012, 12, 30773083.

54 B. M. Venkatesan and R. Bashir, Nat. Nanotechnol., 2011, 6, 615-624.

55 B. N. Miles, A. P. Ivanov, K. A. Wilson, F. Dogan, D. Japrung and J. B. Edel, Chem. Soc. Rev., 2013, 42, 15-28.

56 J. Clarke, H.-C. Wu, L. Jayasinghe, A. Patel, S. Reid and H. Bayley, Nat. Nanotechnol., 2009, 4, 265-270.

57 G. M. Cherf, K. R. Lieberman, H. Rashid, C. E. Lam, K. Karplus and M. Akeson, Nat. Biotechnol., 2012, 30, 344348.

58 E. A. Manrao, I. M. Derrington, A. H. Laszlo, K. W. Langford, M. K. Hopper, N. Gillgren, M. Pavlenok, M. Niederweis and J. H. Gundlach, Nat. Biotechnol., 2012, 30, 349-353.

59 N. C. Seeman, Annu. Rev. Biochem., 2010, 79, 65-87.

60 N. C. Seeman, Nature, 2003, 421, 427-431.

61 P. W. K. Rothemund, Nature, 2006, 440, 297-302.

62 Y. Ke, L. L. Ong, W. M. Shih and P. Yin, Science, 2012, 338, 1177-1183.

63 T. G. Drummond, M. G. Hill and J. K. Barton, Nat. Biotechnol., 2003, 21, 1192-1199.

64 I. Willner, B. Willner and R. Tel-Vered, Electroanalysis, 2011, 23, 13-28.

65 Y. Wen, H. Pei, Y. Shen, J. Xi, M. Lin, N. Lu, X. Shen, J. Li and C. Fan, Sci. Rep., 2012, 2, 867.

66 J. Zhang, R. Lao, S. Song, Z. Yan and C. Fan, Anal. Chem., 2008, 80, 9029-9033.

67 R. Freeman, Y. Li, R. Tel-Vered, E. Sharon, J. Elbaz and I. Willner, Analyst, 2009, 134, 653-656.

68 Y. Lu, X. Li, L. Zhang, P. Yu, L. Su and L. Mao, Anal. Chem., 2008, 80, 1883-1890.

69 O. C. Farokhzad and R. Langer, ACS Nano, 2009, 3, 16-20.

70 Y.-B. Lim, K.-S. Moon and M. Lee, Chem. Soc. Rev., 2009, 38, 925-934.

71 H. Cui, M. J. Webber and S. I. Stupp, Biopolymers, 2010, 94, 1-18. 
72 J. B. Matson and S. I. Stupp, Chem. Commun., 2012, 48, 26-33. 73 E. Gazit, Chem. Soc. Rev., 2007, 36, 1263-1269.

74 M. Antonietti and S. Förster, Adv. Mater., 2003, 15, 13231333.

75 Y. Namiki, T. Fuchigami, N. Tada, R. Kawamura, S. Matsunuma, Y. Kitamoto and M. Nakagawa, Acc. Chem. Res., 2011, 44, 1080-1093.

76 Y. Zhou and T. Shimizu, Chem. Mater., 2007, 20, 625-633.

77 M. Kwak and A. Herrmann, Chem. Soc. Rev., 2011, 40, 57455755.

78 H. Wang, R. Yang, L. Yang and W. Tan, ACS Nano, 2009, 3, 2451-2460.

79 N. C. Seeman, Mol. Biotechnol., 2007, 37, 246-257.

80 A. Kuzuya and M. Komiyama, Nanoscale, 2010, 2, 310-322. 81 Y.-B. Lim and M. Lee, Org. Biomol. Chem., 2007, 5, 401-405. 82 M. Numata and S. Shinkai, Self-Assembled Polysaccharide Nanotubes Generated from $\beta$-1,3-Glucan Polysaccharides, in Self-Assembled Nanomaterials II, ed. T. Shimizu, Springer, Berlin, Heidelberg 2008, vol. 220, ch. 145, pp. 65-121.

83 T. Shimizu, J. Polym. Sci., Part A: Polym. Chem., 2006, 44, 5137-5152.

84 N. C. Reichardt, M. Martin-Lomas and S. Penades, Chem. Soc. Rev., 2013, 42, 4358-4376.

85 M. Numata and S. Shinkai, Chem. Commun., 2011, 47, 19611975.

86 Y. Ma, R. J. Nolte and J. J. Cornelissen, Adv. Drug Delivery Rev., 2012, 64, 811-825.

87 O. Pornillos, B. K. Ganser-Pornillos and M. Yeager, Nature, 2011, 469, 424-427.

88 L. W. Seymour and A. J. Thrasher, Nat. Biotechnol., 2012, 30, 588-593.

89 J. A. Hoxie and C. H. June, Cold Spring Harbor Perspect. Med., 2012, 2, DOI: 10.1101/cshperspect.a007179.

90 I. J. Minten, Y. Ma, M. A. Hempenius, G. J. Vancso, R. J. M. Nolte and J. J. L. M. Cornelissen, Org. Biomol. Chem., 2009, 7, 4685-4688.

91 F. M. Brunel, J. D. Lewis, G. Destito, N. F. Steinmetz, M. Manchester, H. Stuhlmann and P. E. Dawson, Nano Lett., 2010, 10, 1093-1097.

92 I. Yildiz, S. Shukla and N. F. Steinmetz, Curr. Opin. Biotechnol., 2011, 22, 901-908.

93 B. Lamarre and M. G. Ryadnov, Macromol. Biosci., 2011, 11, 503-513.

94 L. Lee, Z. Niu and Q. Wang, Nano Res., 2009, 2, 349-364.

95 K. Matsuura, K. Murasato and N. Kimizuka, J. Am. Chem. Soc., 2005, 127, 10148-10149.

96 R. Zandi, D. Reguera, R. F. Bruinsma, W. M. Gelbart and J. Rudnick, Proc. Natl. Acad. Sci. U. S. A., 2004, 101, 1555615560.

97 K. Matsuura, K. Watanabe, T. Matsuzaki, K. Sakurai and N. Kimizuka, Angew. Chem., Int. Ed., 2010, 49, 9662-9665.

98 S. Raman, G. Machaidze, A. Lustig, U. Aebi and P. Burkhard, Nanomedicine: Nanotechnology, Biology and Medicine, 2006, 2, 95-102.

99 T. A. P. F. Pimentel, Z. Yan, S. A. Jeffers, K. V. Holmes, R. S. Hodges and P. Burkhard, Chem. Biol. Drug Des., 2009, 73, 53-61.
100 F. Boato, R. M. Thomas, A. Ghasparian, A. Freund-Renard, K. Moehle and J. A. Robinson, Angew. Chem., Int. Ed., 2007, 46, 9015-9018.

101 D. Fujita, K. Suzuki, S. Sato, M. Yagi-Utsumi, Y. Yamaguchi, N. Mizuno, T. Kumasaka, M. Takata, M. Noda, S. Uchiyama, K. Kato and M. Fujita, Nat. Commun., 2012, 3, 1093.

102 Y.-b. Lim, E. Lee, Y.-R. Yoon, M. Ä. Lee and M. Lee, Angew. Chem., Int. Ed., 2008, 47, 4525-4528.

103 Y. Aoyama, Chem.-Eur. J., 2004, 10, 588-593.

104 A. D. Bangham, M. M. Standish and J. C. Watkins, J. Mol. Biol., 1965, 13, 238-252.

105 T. M. Allen and P. R. Cullis, Adv. Drug Delivery Rev., 2013, 65, 36-48.

106 M. L. Tan, P. F. M. Choong and C. R. Dass, Peptides, 2010, 31, 184-193.

107 T. Lian and R. J. Y. Ho, J. Pharm. Sci., 2001, 90, 667-680.

108 T. M. Allen and P. R. Cullis, Science, 2004, 303, 1818-1822. 109 Y. Malam, M. Loizidou and A. M. Seifalian, Trends Pharmacol. Sci., 2009, 30, 592-599.

110 Y. Barenholz, J. Controlled Release, 2012, 160, 117-134.

111 S. Bhattacharya and A. Bajaj, Chem. Commun., 2009, 46324656.

112 M. A. Mintzer and E. E. Simanek, Chem. Rev., 2008, 109, 259-302.

113 J.-P. Behr, Bioconjugate Chem., 1994, 5, 382-389.

114 X.-X. Zhang, T. J. McIntosh and M. W. Grinstaff, Biochimie, 2012, 94, 42-58.

115 K. Ewert, H. M. Evans, A. Ahmad, N. L. Slack, A. J. Lin, A. Martin-Herranz and C. R. Safinya, Lipoplex Structures and Their Distinct Cellular Pathways, in Advances in Genetics, ed. M.-C. H. Leaf Huang and W. Ernst, Academic Press, 2005, vol. 53, pp. 119-155.

116 G. Caracciolo and H. Amenitsch, Eur. Biophys. J., 2012, 41, 815-829.

117 A. Bilalov, U. Olsson and B. Lindman, Soft Matter, 2012, 8, 11022-11033.

118 K. A. Whitehead, R. Langer and D. G. Anderson, Nat. Rev. Drug Discovery, 2009, 8, 129-138.

119 L. C. Gomes-da-Silva, N. A. Fonseca, V. Moura, M. C. Pedroso de Lima, S. Simões and J. N. Moreira, Acc. Chem. Res., 2012, 45, 1163-1171.

120 M. Morille, C. Passirani, A. Vonarbourg, A. Clavreul and J.-P. Benoit, Biomaterials, 2008, 29, 3477-3496.

121 A. Akinc, A. Zumbuehl, M. Goldberg, E. S. Leshchiner, V. Busini, N. Hossain, S. A. Bacallado, D. N. Nguyen, J. Fuller, R. Alvarez, A. Borodovsky, T. Borland, R. Constien, A. de Fougerolles, J. R. Dorkin, K. Narayanannair Jayaprakash, M. Jayaraman, M. John, V. Koteliansky, M. Manoharan, L. Nechev, J. Qin, T. Racie, D. Raitcheva, K. G. Rajeev, D. W. Y. Sah, J. Soutschek, I. Toudjarska, H.-P. Vornlocher, T. S. Zimmermann, R. Langer and D. G. Anderson, Nat. Biotechnol., 2008, 26, 561-569.

122 T. S. Zimmermann, A. C. H. Lee, A. Akinc, B. Bramlage, D. Bumcrot, M. N. Fedoruk, J. Harborth, J. A. Heyes, L. B. Jeffs, M. John, A. D. Judge, K. Lam, K. McClintock, L. V. Nechev, L. R. Palmer, T. Racie, I. Röhl, S. Seiffert, 
S. Shanmugam, V. Sood, J. Soutschek, I. Toudjarska, A. J. Wheat, E. Yaworski, W. Zedalis, V. Koteliansky, M. Manoharan, H.-P. Vornlocher and I. MacLachlan, Nature, 2006, 441, 111-114.

123 Y. Sato, K. Murase, J. Kato, M. Kobune, T. Sato, Y. Kawano, R. Takimoto, K. Takada, K. Miyanishi, T. Matsunaga, T. Takayama and Y. Niitsu, Nat. Biotechnol., 2008, 26, 431-442.

124 S. Deshayes, M. Morris, F. Heitz and G. Divita, Adv. Drug Delivery Rev., 2008, 60, 537-547.

125 A. Thomas, L. Lins, G. Divita and R. Brasseur, Biochim. Biophys. Acta, Biomembr., 2010, 1798, 2217-2222.

126 F. Heitz, M. C. Morris and G. Divita, Br. J. Pharmacol., 2009, 157, 195-206.

127 Y. Yue and C. Wu, Biomater. Sci., 2013, 1, 152-170.

128 K. Miyata, N. Nishiyama and K. Kataoka, Chem. Soc. Rev., 2012, 41, 2562-2574.

129 Y. Vachutinsky and K. Kataoka, Isr. J. Chem., 2010, 50, 175184.

130 K. Itaka, K. Yamauchi, A. Harada, K. Nakamura, H. Kawaguchi and K. Kataoka, Biomaterials, 2003, 24, 4495-4506.

131 K. Osada, H. Oshima, D. Kobayashi, M. Doi, M. Enoki, Y. Yamasaki and K. Kataoka, J. Am. Chem. Soc., 2010, 132, 12343-12348.

132 R. J. Christie, Y. Matsumoto, K. Miyata, T. Nomoto, S. Fukushima, K. Osada, J. Halnaut, F. Pittella, H. J. Kim, N. Nishiyama and K. Kataoka, ACS Nano, 2012, 6, 51745189.

133 K. Sakurai, K. Uezu, M. Numata, T. Hasegawa, C. Li, K. Kaneko and S. Shinkai, Chem. Commun., 2005, 43834398.

134 A. Hernandez-Garcia, M. W. T. Werten, M. C. Stuart, F. A. de Wolf and R. de Vries, Small, 2012, 8, 3491-3501.

135 X. Jiang, W. Qu, D. Pan, Y. Ren, J.-M. Williford, H. Cui, E. Luijten and H.-Q. Mao, Adv. Mater., 2013, 25, 227-232.

136 M. E. Davis, Mol. Pharmaceutics, 2009, 6, 659-668.

137 M. E. Davis, J. E. Zuckerman, C. H. J. Choi, D. Seligson, A. Tolcher, C. A. Alabi, Y. Yen, J. D. Heidel and A. Ribas, Nature, 2010, 464, 1067-1070.

138 J. S. Lee and J. Feijen, J. Controlled Release, 2012, 161, 473483.

139 V. Percec, D. A. Wilson, P. Leowanawat, C. J. Wilson, A. D. Hughes, M. S. Kaucher, D. A. Hammer, D. H. Levine, A. J. Kim, F. S. Bates, K. P. Davis, T. P. Lodge, M. L. Klein, R. H. DeVane, E. Aqad, B. M. Rosen, A. O. Argintaru, M. J. Sienkowska, K. Rissanen, S. Nummelin and J. Ropponen, Science, 2010, 328, 1009-1014.

140 S. Rana, A. Bajaj, R. Mout and V. M. Rotello, Adv. Drug Delivery Rev., 2012, 64, 200-216.

141 E. C. Dreaden, M. A. Mackey, X. Huang, B. Kang and M. A. El-Sayed, Chem. Soc. Rev., 2011, 40, 3391-3404.

142 E. C. Dreaden, A. M. Alkilany, X. Huang, C. J. Murphy and M. A. El-Sayed, Chem. Soc. Rev., 2012, 41, 2740-2779.

143 C. K. Kim, P. Ghosh, C. Pagliuca, Z.-J. Zhu, S. Menichetti and V. M. Rotello, J. Am. Chem. Soc., 2009, 131, 1360-1361.
144 C. Park, H. Youn, H. Kim, T. Noh, Y. H. Kook, E. T. Oh, H. J. Park and C. Kim, J. Mater. Chem., 2009, 19, 2310-2315. 145 D. A. Giljohann, D. S. Seferos, W. L. Daniel, M. D. Massich, P. C. Patel and C. A. Mirkin, Angew. Chem., Int. Ed., 2010, 49, 3280-3294.

146 D. Zheng, D. A. Giljohann, D. L. Chen, M. D. Massich, X.-Q. Wang, H. Iordanov, C. A. Mirkin and A. S. Paller, Proc. Natl. Acad. Sci. U. S. A., 2012, 109, 11975-11980.

147 M. W. Ambrogio, C. R. Thomas, Y. Zhao, J. I. Zink and J. F. Stoddart, Acc. Chem. Res., 2012, 44, 903-913.

148 C. R. Thomas, D. P. Ferris, J.-H. Lee, E. Choi, M. H. Cho, E. S. Kim, J. F. Stoddart, J.-S. Shin, J. Cheon and J. I. Zink, J. Am. Chem. Soc., 2010, 132, 10623-10625.

149 K. Kataoka, A. Harada and Y. Nagasaki, Adv. Drug Delivery Rev., 2001, 47, 113-131.

150 R. K. O'Reilly, C. J. Hawker and K. L. Wooley, Chem. Soc. Rev., 2006, 35, 1068-1083.

151 S. V. Aathimanikandan, E. N. Savariar and S. Thayumanavan, J. Am. Chem. Soc., 2005, 127, 14922-14929.

152 E. Fleige, M. A. Quadir and R. Haag, Adv. Drug Delivery Rev., 2012, 64, 866-884.

153 J. Xu and S. Liu, Soft Matter, 2008, 4, 1745-1749.

154 T. Chen, C. S. Wu, E. Jimenez, Z. Zhu, J. G. Dajac, M. You, D. Han, X. Zhang and W. Tan, Angew. Chem., Int. Ed., 2013, 52, 2012-2016.

155 A. M. Rush, M. P. Thompson, E. T. Tatro and N. C. Gianneschi, ACS Nano, 2013, 7, 1379-1387.

156 S. E. A. Gratton, P. A. Ropp, P. D. Pohlhaus, J. C. Luft, V. J. Madden, M. E. Napier and J. M. DeSimone, Proc. Natl. Acad. Sci. U. S. A., 2008, 105, 11613-11618.

157 Y. Geng, P. Dalhaimer, S. Cai, R. Tsai, M. Tewari, T. Minko and D. E. Discher, Nat. Nanotechnol., 2007, 2, 249-255.

158 J. B. Matson, M. J. Webber, V. K. Tamboli, B. Weber and S. I. Stupp, Soft Matter, 2012, 8, 6689-6692.

159 J. B. Matson and S. I. Stupp, Chem. Commun., 2011, 47, 7962-7964.

160 S. Soukasene, D. J. Toft, T. J. Moyer, H. Lu, H.-K. Lee, S. M. Standley, V. L. Cryns and S. I. Stupp, ACS Nano, 2011, 5, 9113-9121.

161 M. J. Webber, C. J. Newcomb, R. Bitton and S. I. Stupp, Soft Matter, 2011, 7, 9665-9672.

162 D. J. Toft, T. J. Moyer, S. M. Standley, Y. Ruff, A. Ugolkov, S. I. Stupp and V. L. Cryns, ACS Nano, 2012, 6, 7956-7965.

163 A. G. Cheetham, P. Zhang, Y.-A. Lin, L. L. Lock and H. Cui, J. Am. Chem. Soc., 2013, 135, 2907-2910.

164 D. Pantarotto, R. Singh, D. McCarthy, M. Erhardt, J. Briand, M. Prato, K. Kostarelos and A. Bianco, Angew. Chem., Int. Ed., 2004, 43, 5242-5246.

165 Z. Liu, S. M. Tabakman, Z. Chen and H. Dai, Nat. Protocols, 2009, 4, 1372-1381.

166 A. Bianco, K. Kostarelos and M. Prato, Curr. Opin. Chem. Biol., 2005, 9, 674-679.

167 Z. Liu, X. Sun, N. Nakayama-Ratchford and H. Dai, ACS Nano, 2007, 1, 50-56.

168 Z. Liu, A. C. Fan, K. Rakhra, S. Sherlock, A. Goodwin, X. Chen, Q. Yang, D. W. Felsher and H. Dai, Angew. Chem., Int. Ed., 2009, 48, 7668-7672. 
169 M. Hamidi, A. Azadi and P. Rafiei, Adv. Drug Delivery Rev., 2008, 60, 1638-1649.

170 T. R. Hoare and D. S. Kohane, Polymer, 2008, 49, 1993-2007.

171 A. Altunbas and D. Pochan, Peptide-Based and PolypeptideBased Hydrogels for Drug Delivery and Tissue Engineering, in Peptide-Based Materials, ed. T. Deming, Springer, Berlin, Heidelberg, 2012, vol. 310, ch. 206, pp. 135-167.

172 A. Altunbas, S. J. Lee, S. A. Rajasekaran, J. P. Schneider and D. J. Pochan, Biomaterials, 2011, 32, 5906-5914.

173 Y. Kim, M. Park and S. Song, ACS Nano, 2012, 6, 5757-5766. 174 D. Bhatia, S. Sharma and Y. Krishnan, Curr. Opin. Biotechnol., 2011, 22, 475-484.

175 Y.-X. Zhao, A. Shaw, X. Zeng, E. Benson, A. M. Nyström and B. Högberg, ACS Nano, 2012, 6, 8684-8691.

176 E. S. Andersen, M. Dong, M. M. Nielsen, K. Jahn, R. Subramani, W. Mamdouh, M. M. Golas, B. Sander, H. Stark, C. L. P. Oliveira, J. S. Pedersen, V. Birkedal, F. Besenbacher, K. V. Gothelf and J. Kjems, Nature, 2009, 459, 73-76.

177 S. M. Douglas, I. Bachelet and G. M. Church, Science, 2012, 335, 831-834.

178 T. Dvir, B. P. Timko, D. S. Kohane and R. Langer, Nat. Nanotechnol., 2011, 6, 13-22.

179 E. Engel, A. Michiardi, M. Navarro, D. Lacroix and J. A. Planell, Trends Biotechnol., 2008, 26, 39-47.

180 C. Cha, W. B. Liechty, A. Khademhosseini and N. A. Peppas, ACS Nano, 2012, 6, 9353-9358.

181 S. I. Stupp, Nano Lett., 2010, 10, 4783-4786.

182 T. Aida, E. W. Meijer and S. I. Stupp, Science, 2012, 335, 813-817.

183 J. B. Matson, R. H. Zha and S. I. Stupp, Curr. Opin. Solid State Mater. Sci., 2011, 15, 225-235.

184 N. Stephanopoulos, J. H. Ortony and S. I. Stupp, Acta Mater., 2013, 61, 912-930.

185 E. C. Wu, S. Zhang and C. A. E. Hauser, Adv. Funct. Mater., 2012, 22, 456-468.

186 Z. Luo and S. Zhang, Chem. Soc. Rev., 2012, 41, 4736-4754.

187 V. M. Tysseling-Mattiace, V. Sahni, K. L. Niece, D. Birch, C. Czeisler, M. G. Fehlings, S. I. Stupp and J. A. Kessler, J. Neurosci., 2008, 28, 3814-3823.

188 A. Mata, Y. Geng, K. J. Henrikson, C. Aparicio, S. R. Stock, R. L. Satcher and S. I. Stupp, Biomaterials, 2010, 31, 60046012.

189 R. N. Shah, N. A. Shah, M. M. Del Rosario Lim, C. Hsieh, G. Nuber and S. I. Stupp, Proc. Natl. Acad. Sci. U. S. A., 2010, 107, 3293-3298.

190 K. Rajangam, H. A. Behanna, M. J. Hui, X. Han, J. F. Hulvat, J. W. Lomasney and S. I. Stupp, Nano Lett., 2006, 6, 20862090.

191 M. J. Webber, X. Han, S. N. Prasanna Murthy, K. Rajangam, S. I. Stupp and J. W. Lomasney, J. Tissue Eng. Regener. Med., 2010, 4, 600-610.

192 J. C. Stendahl, L.-J. Wang, L. W. Chow, D. B. Kaufman and S. I. Stupp, Transplantation, 2008, 86, 478-481.

193 Z. Huang, T. D. Sargeant, J. F. Hulvat, A. Mata, P. Bringas, C.-Y. Koh, S. I. Stupp and M. L. Snead, J. Bone Miner. Res., 2008, 23, 1995-2006.
194 M. J. Webber, J. Tongers, C. J. Newcomb, K.-T. Marquardt, J. Bauersachs, D. W. Losordo and S. I. Stupp, Proc. Natl. Acad. Sci. U. S. A., 2011, 108, 13438-13443.

195 S. Zhang, M. A. Greenfield, A. Mata, L. C. Palmer, R. Bitton, J. R. Mantei, C. Aparicio, M. O. de la Cruz and S. I. Stupp, Nat. Mater., 2010, 9, 594-601.

196 M. T. McClendon and S. I. Stupp, Biomaterials, 2012, 33, 5713-5722.

197 R. M. Capito, H. S. Azevedo, Y. S. Velichko, A. Mata and S. I. Stupp, Science, 2008, 319, 1812-1816.

198 L. W. Chow, R. Bitton, M. J. Webber, D. Carvajal, K. R. Shull, A. K. Sharma and S. I. Stupp, Biomaterials, 2011, 32, 1574-1582.

199 Y. Yang, U. Khoe, X. Wang, A. Horii, H. Yokoi and S. Zhang, Nano Today, 2009, 4, 193-210.

200 A. Horii, X. Wang, F. Gelain and S. Zhang, PLoS One, 2007, 2, e190.

201 L. E. R. O'Leary, J. A. Fallas, E. L. Bakota, M. K. Kang and J. D. Hartgerink, Nat. Chem., 2011, 3, 821-828.

202 Organic Electronics: Materials, Manufacturing and Applications, ed. H. Klauk, Wiley-VCH Verlag \& Co. KGaA, Weinheim, 2006.

203 Organic Electronics, ed. G. Meller and T. Grasser, Advances in Polymer Science, vol. 223, 2010.

204 Semiconducting Polymers: Chemistry, Physics and Engineering, ed. G. Hadziioannou and G. G. Malliaras, Wiley-VCH Verlag \& Co. KGaA, Weinheim, 2nd edn, 2006.

205 Molecular and Supramolecular Information Processing, ed. E. Katz, Wiley-VCH Verlag \& Co. KGaA, Weinheim, 2012.

206 Nanoelectronics and Information Technology, ed. R. Waser, Wiley-VCH Verlag \& Co. KGaA, Weinheim, 3rd edn, 2012.

207 U. Shinya, M. Takehiko and T. Toshihiro, Sci. Technol. Adv. Mater., 2009, 10, 020301.

208 Y. Yamamoto, Sci. Technol. Adv. Mater., 2012, 13, 33001.

209 E. Moulin, J.-J. Cid and N. Giuseppone, Adv. Mater., 2013, 25, 477-487.

210 S. S. Babu, S. Prasanthkumar and A. Ajayaghosh, Angew. Chem., Int. Ed., 2012, 51, 1766-1776.

211 M. Hasegawa and M. Iyoda, Chem. Soc. Rev., 2010, 39, 24202427.

212 E. Moulin, F. Niess, M. Maaloum, E. Buhler, I. Nyrkova and N. Giuseppone, Angew. Chem., Int. Ed., 2010, 49, 6974-6978.

213 V. Faramarzi, F. Niess, E. Moulin, M. Maaloum, J.-F. Dayen, J.-B. Beaufrand, S. Zanettini, B. Doudin and N. Giuseppone, Nat. Chem., 2012, 4, 485-490.

214 E. Moulin, F. Niess, G. Fuks, N. Jouault, E. Buhler and N. Giuseppone, Nanoscale, 2012, 4, 6748-6751.

215 N. Giuseppone, Acc. Chem. Res., 2012, 45, 2178-2188.

216 M. Hasegawa, H. Enozawa, Y. Kawabata and M. Iyoda, J. Am. Chem. Soc., 2007, 129, 3072-3073.

217 J. Puigmartí-Luis, V. Laukhin, A. Pérez del Pino, J. VidalGancedo, C. Rovira, E. Laukhina and D. B. Amabilino, Angew. Chem., Int. Ed., 2007, 46, 238-241.

218 S. Ahn, Y. Kim, S. Beak, S. Ishimoto, H. Enozawa, E. Isomura, M. Hasegawa, M. Iyoda and Y. Park, J. Mater. Chem., 2010, 20, 10817-10823.

219 S. Sengupta, D. Ebeling, S. Patwardhan, X. Zhang, H. von Berlepsch, C. Böttcher, V. Stepanenko, S. Uemura, 
C. Hentschel, H. Fuchs, F. C. Grozema, L. D. A. Siebbeles, A. R. Holzwarth, L. Chi and F. Würthner, Angew. Chem., Int. Ed., 2012, 51, 6378-6382.

220 Y. Yamamoto, G. Zhang, W. Jin, T. Fukushima, N. Ishii, A. Saeki, S. Seki, S. Tagawa, T. Minari, K. Tsukagoshi and T. Aida, Proc. Natl. Acad. Sci. U. S. A., 2009, 106, 2105121056.

221 J. E. Kroeze, T. J. Savenije and J. M. Warman, J. Am. Chem. Soc., 2004, 126, 7608-7618.

222 J. E. Kroeze, T. J. Savenije, M. J. W. Vermeulen and J. M. Warman, J. Phys. Chem. B, 2003, 107, 7696-7705.

223 S. Yagai, T. Kinoshita, Y. Kikkawa, T. Karatsu, A. Kitamura, Y. Honsho and S. Seki, Chem.-Eur. J., 2009, 15, 93209324.

224 J. M. Warman and A. M. Van De Craats, Mol. Cryst. Liq. Cryst., 2003, 396, 41-72.

225 J. M. Warman, M. P. de Haas, G. Dicker, F. C. Grozema, J. Piris and M. G. Debije, Chem. Mater., 2004, 16, 4600-4609.

226 Y. Che, A. Datar, X. Yang, T. Naddo, J. Zhao and L. Zang, J. Am. Chem. Soc., 2007, 129, 6354-6355.

227 C. Wang, H. Dong, W. Hu, Y. Liu and D. Zhu, Chem. Rev., 2012, 112, 2208-2267.

228 M. Mas-Torrent and C. Rovira, Chem. Rev., 2011, 111, 48334856.

229 A. Facchetti, Mater. Today, 2007, 10, 28-37.

230 D. M. Bassani, L. Jonusauskaite, A. Lavie-Cambot, N. D. McClenaghan, J.-L. Pozzo, D. Ray and G. Vives, Coord. Chem. Rev., 2010, 254, 2429-2445.

231 H. Imahori, T. Umeyama, K. Kurotobi and Y. Takano, Chem. Commun., 2012, 48, 4032-4045.

232 B. R. Kaafarani, Chem. Mater., 2011, 23, 378-396.

233 F. S. Kim, G. Ren and S. A. Jenekhe, Chem. Mater., 2011, 23, 682-732.

234 J.-P. Hong, M.-C. Um, S.-R. Nam, J.-I. Hong and S. Lee, Chem. Commun., 2009, 310-312.

235 R. C. Savage, E. Orgiu, J. M. Mativetsky, W. Pisula, T. Schnitzler, C. L. Eversloh, C. Li, K. Müllen and P. Samorì, Nanoscale, 2012, 4, 2387-2393.

236 J. H. Oh, H. W. Lee, S. Mannsfeld, R. M. Stoltenberg, E. Jung, Y. W. Jin, J. M. Kim, J.-B. Yoo and Z. Bao, Proc. Natl. Acad. Sci. U. S. A., 2009, 106, 6065-6070.

237 H. Dong, S. Jiang, L. Jiang, Y. Liu, H. Li, W. Hu, E. Wang, S. Yan, Z. Wei, W. Xu and X. Gong, J. Am. Chem. Soc., 2009, 131, 17315-17320.

238 P. Jonkheijm, N. Stutzmann, Z. Chen, D. M. de Leeuw, E. W. Meijer, A. P. H. J. Schenning and F. Würthner, $J$. Am. Chem. Soc., 2006, 128, 9535-9540.

239 A. L. Briseno, S. C. B. Mannsfeld, C. Reese, J. M. Hancock, Y. Xiong, S. A. Jenekhe, Z. Bao and Y. Xia, Nano Lett., 2007, 7, 2847-2853.

240 A. L. Briseno, S. C. B. Mannsfeld, P. J. Shamberger, F. S. Ohuchi, Z. Bao, S. A. Jenekhe and Y. Xia, Chem. Mater., 2008, 20, 4712-4719.

241 Y. Zhou, W.-J. Liu, Y. Ma, H. Wang, L. Qi, Y. Cao, J. Wang and J. Pei, J. Am. Chem. Soc., 2007, 129, 12386-12387.

242 Y. Zhou, T. Lei, L. Wang, J. Pei, Y. Cao and J. Wang, Adv. Mater., 2010, 22, 1484-1487.
243 W. Jiang, Y. Zhou, H. Geng, S. Jiang, S. Yan, W. Hu, Z. Wang, Z. Shuai and J. Pei, J. Am. Chem. Soc., 2011, 133, 1-3.

244 D. H. Kim, B.-L. Lee, H. Moon, H. M. Kang, E. J. Jeong, J.-i. Park, K.-m. Han, S. Lee, B. W. Yoo, B. W. Koo, J. Y. Kim, W. H. Lee, K. Cho, H. A. Becerril and Z. Bao, J. Am. Chem. Soc., 2009, 131, 6124-6132.

245 H. N. Tsao, W. Pisula, Z. Liu, W. Osikowicz, W. R. Salaneck and K. Müllen, Adv. Mater., 2008, 20, 2715-2719.

246 S. Dong, H. Tian, D. Song, Z. Yang, D. Yan, Y. Geng and F. Wang, Chem. Commun., 2009, 3086-3088.

247 H. Hayashi, W. Nihashi, T. Umeyama, Y. Matano, S. Seki, Y. Shimizu and H. Imahori, J. Am. Chem. Soc., 2011, 133, 10736-10739.

248 S. Tiwari and N. C. Greenham, Opt. Quantum Electron., 2009, 41, 69-89.

249 Y. Gao, P. Ma, Y. Chen, Y. Zhang, Y. Bian, X. Li, J. Jiang and C. Ma, Inorg. Chem., 2009, 48, 45-54.

250 B. Geffroy, P. le Roy and C. Prat, Polym. Int., 2006, 55, 572582.

251 K. T. Kamtekar, A. P. Monkman and M. R. Bryce, Adv. Mater., 2010, 22, 572-582.

252 L. Xiao, Z. Chen, B. Qu, J. Luo, S. Kong, Q. Gong and J. Kido, Adv. Mater., 2011, 23, 926-952.

253 M. C. Gather, A. Köhnen and K. Meerholz, Adv. Mater., 2011, 23, 233-248.

254 S. Chen, L. Deng, J. Xie, L. Peng, L. Xie, Q. Fan and W. Huang, Adv. Mater., 2010, 22, 5227-5239.

255 D. Ray, C.-K. Liang, N. D. McClenaghan and D. M. Bassani, Curr. Phys. Chem., 2011, 1, 169-180.

256 R. D. Costa, E. Ortí, H. J. Bolink, F. Monti, G. Accorsi and N. Armaroli, Angew. Chem., Int. Ed., 2012, 51, 8178-8211.

257 G. M. Farinola and R. Ragni, Chem. Soc. Rev., 2011, 40, 3467-3482.

258 T. Hu, L. He, L. Duan and Y. Qiu, J. Mater. Chem., 2012, 22, 4206.

259 C. Ulbricht, B. Beyer, C. Friebe, A. Winter and U. S. Schubert, Adv. Mater., 2009, 21, 4418-4441.

260 C. Giansante, G. Raffy, C. Schäfer, H. Rahma, M.-T. Kao, A. G. L. Olive and A. Del Guerzo, J. Am. Chem. Soc., 2011, 133, 316-325.

261 C. A. Strassert, C.-H. Chien, M. D. Galvez Lopez, D. Kourkoulos, D. Hertel, K. Meerholz and L. De Cola, Angew. Chem., Int. Ed., 2011, 50, 946-950.

262 J. Luo, T. Lei, L. Wang, Y. Ma, Y. Cao, J. Wang and J. Pei, J. Am. Chem. Soc., 2009, 131, 2076-2077.

263 A. Winter, C. Friebe, M. D. Hager and U. S. Schubert, Macromol. Rapid Commun., 2008, 29, 1679-1686.

264 R. Abbel, C. Grenier, M. J. Pouderoijen, J. W. Stouwdam, P. E. L. G. Leclère, R. P. Sijbesma, E. W. Meijer and A. P. H. J. Schenning, J. Am. Chem. Soc., 2009, 131, 833-843.

265 Y.-L. Chu, C.-C. Cheng, Y.-C. Yen and F.-C. Chang, Adv. Mater., 2012, 24, 1894-1898.

266 N. Delbosc, M. Reynes, O. J. Dautel, G. Wantz, J.-P. LèrePorte and J. E. Moreau, Chem. Mater., 2010, 22, 5258-5270.

267 X.-H. Zhao, G.-H. Xie, Z.-D. Liu, W.-J. Li, M.-D. Yi, L.-H. Xie, C.-P. Hu, R. Zhu, Q. Zhao, Y. Zhao, J.-F. Zhao, Y. Qian and W. Huang, Chem. Commun., 2012, 48, 3854-3856. 
268 D. Tordera, S. Meier, M. Lenes, R. D. Costa, E. Ortí, W. Sarfert and H. J. Bolink, Adv. Mater., 2012, 24, 897-900. 269 H.-C. Su, H.-F. Chen, Y.-C. Shen, C.-T. Liao and K.-T. Wong, J. Mater. Chem., 2011, 21, 9653.

270 S. Brovelli, F. Meinardi, G. Winroth, O. Fenwick, G. Sforazzini, M. J. Frampton, L. Zalewski, J. a. Levitt, F. Marinello, P. Schiavuta, K. Suhling, H. L. Anderson and F. Cacialli, Adv. Funct. Mater., 2010, 20, 272-280.

271 A. W. Hains, Z. Liang, M. A. Woodhouse and B. A. Gregg, Chem. Rev., 2010, 110, 6689-6735.

272 S. Günes, H. Neugebauer and N. S. Sariciftci, Chem. Rev., 2007, 107, 1324-1338.

273 Y. Lin, Y. Li and X. Zhan, Chem. Soc. Rev., 2012, 41, 42454272.

274 A. Mishra and P. Bäuerle, Angew. Chem., Int. Ed., 2012, 51, 2020-2067.

275 M. Grätzel, J. Photochem. Photobiol., C, 2003, 4, 145-153.

276 T. M. Clarke and J. R. Durrant, Chem. Rev., 2010, 110, 67366767.

277 W. Chen, M. P. Nikiforov and S. B. Darling, Energy Environ. Sci., 2012, 5, 8045-8074.

278 A. Facchetti, Chem. Mater., 2011, 23, 733-758.

279 G. Bottari, O. Trukhina, M. Ince and T. Torres, Coord. Chem. Rev., 2012, 256, 2453-2477.

280 D. González-Rodríguez and A. P. H. J. Schenning, Chem. Mater., 2011, 23, 310-325.

281 M. Wang and F. Wudl, J. Mater. Chem., 2012, 22, 2429724314.

282 Y. Hizume, K. Tashiro, R. Charvet, Y. Yamamoto, A. Saeki, S. Seki and T. Aida, J. Am. Chem. Soc., 2010, 132, 66286629.

283 K. Sugiyasu, S.-i. Kawano, N. Fujita and S. Shinkai, Chem. Mater., 2008, 20, 2863-2865.

284 A. a. Gorodetsky, C.-Y. Chiu, T. Schiros, M. Palma, M. Cox, Z. Jia, W. Sattler, I. Kymissis, M. Steigerwald and C. Nuckolls, Angew. Chem., Int. Ed., 2010, 49, 7909-7912.

285 S. J. Kang, J. B. Kim, C.-Y. Chiu, S. Ahn, T. Schiros, S. S. Lee, K. G. Yager, M. F. Toney, Y.-L. Loo and C. Nuckolls, Angew. Chem., Int. Ed., 2012, 51, 8594-8597.

286 F. Würthner, Z. Chen, F. J. M. Hoeben, P. Osswald, C.-C. You, P. Jonkheijm, J. V. Herrikhuyzen, A. P. H. J. Schenning, P. P. A. M. van der Schoot, E. W. Meijer, E. H. A. Beckers, S. C. J. Meskers and R. A. J. Janssen, J. Am. Chem. Soc., 2004, 126, 10611-10618.

287 R. J. Kumar, J. M. MacDonald, T. B. Singh, L. J. Waddington and A. B. Holmes, J. Am. Chem. Soc., 2011, 133, 8564-8573.

288 T. Hasobe, S. Fukuzumi and P. V. Kamat, Angew. Chem., Int. Ed., 2006, 45, 755-759.

289 Y. Yamamoto, T. Fukushima, Y. Suna, N. Ishii, A. Saeki, S. Seki, S. Tagawa, M. Taniguchi, T. Kawai and T. Aida, Science, 2006, 314, 1761-1764.

290 W. Zhang, W. Jin, T. Fukushima, A. Saeki, S. Seki and T. Aida, Science, 2011, 334, 340-343.

291 A. S. D. Sandanayaka, T. Murakami and T. Hasobe, J. Phys. Chem. C, 2009, 113, 18369-18378.

292 W. W. H. Wong, T. B. Singh, D. Vak, W. Pisula, C. Yan, X. Feng, E. L. Williams, K. L. Chan, Q. Mao, D. J. Jones,
C.-Q. Ma, K. Müllen, P. Bäuerle and A. B. Holmes, Adv. Funct. Mater., 2010, 20, 927-938.

293 W. W. H. Wong, C.-Q. Ma, W. Pisula, A. Mavrinskiy, X. Feng, H. Seyler, D. J. Jones, K. Müllen, P. Bäuerle and A. B. Holmes, Chem.-Eur. J., 2011, 17, 5549-5560.

$294 \mathrm{~W}$. W. H. Wong, J. Subbiah, S. R. Puniredd, B. Purushothaman, W. Pisula, N. Kirby, K. Müllen, D. J. Jones and A. B. Holmes, J. Mater. Chem., 2012, 22, 21131-21137.

295 M. Carrasco-Orozco, W. C. Tsoi, M. O'Neill, M. P. Aldred, P. Vlachos and S. M. Kelly, Adv. Mater., 2006, 18, 1754-1758.

296 X. Zhou, S.-W. Kang, S. Kumar, R. R. Kulkarni, S. Z. D. Cheng and Q. Li, Chem. Mater., 2008, 20, 3551-3553. 297 L. Li, S. W. Kang, J. Harden, Q. Sun, X. Zhou, L. Dai, A. Jakli, S. Kumar and Q. Li, Liq. Cryst., 2008, 35, 233-239.

298 N. Sakai, R. Bhosale, D. Emery, J. Mareda and S. Matile, J. Am. Chem. Soc., 2010, 132, 6923-6925.

299 R. Bhosale, A. Perez-Velasco, V. Ravikumar, R. S. K. Kishore, O. Kel, A. Gomez-Casado, P. Jonkheijm, J. Huskens, P. Maroni, M. Borkovec, T. Sawada, E. Vauthey, N. Sakai and S. Matile, Angew. Chem., Int. Ed., 2009, 48, 6461-6464.

300 R. Bhosale, N. Sakai and S. Matile, Chem. Soc. Rev., 2010, 39, 138-149.

301 B. J. Walker, A. Dorn, V. Bulović and M. G. Bawendi, Nano Lett., 2011, 11, 2655-2659.

302 M. El Gemayel, M. Treier, C. Musumeci, C. Li, K. Müllen and P. Samori, J. Am. Chem. Soc., 2012, 134, 2429-2433.

303 S. Suresh and D. Arivuoli, Rev. Adv. Mater. Sci., 2012, 30, 243-253.

304 C. J. Strachan, M. Windbergs and H. L. Offerhaus, Int. J. Pharm., 2011, 417, 163-172.

305 H. A. Collins, M. Khurana, E. H. Moriyama, A. Mariampillai, E. Dahlstedt, M. Balaz, M. K. Kuimova, M. Drobizhev, X. D. YangVictor, D. Phillips, A. Rebane, B. C. Wilson and H. L. Anderson, Nat. Photonics, 2008, 2, 420-424.

306 K.-S. Lee, R. H. Kim, D.-Y. Yang and S. H. Park, Prog. Polym. Sci., 2008, 33, 631-681.

307 S. Maruo and J. T. Fourkas, Laser Photonics Rev., 2008, 2, 100-111.

308 T. Verbiest, S. V. Elshocht, M. Kauranen, L. Hellemans, J. Snauwaert, C. Nuckolls, T. J. Katz and A. Persoons, Science, 1998, 282, 913-915.

309 M. Pawlicki, H. A. Collins, R. G. Denning and H. L. Anderson, Angew. Chem., Int. Ed., 2009, 48, 3244-3266. 310 T. Ishi-i, S. Amemori, C. Okamura, K. Yanaga, R. Kuwahara, S. Mataka and K. Kamada, Tetrahedron, 2013, 69, 29-37.

311 J. Pérez-Moreno, I. Asselberghs, K. Song, K. Clays, Y. Zhao, H. Nakanishi, S. Okada, K. Nogi, O.-K. Kim, J. Je, J. Mátrai, M. De Maeyer and M. G. Kuzyk,J. Chem. Phys., 2007, 126, 1-14.

312 L. A. Burke, G. Gonella, F. Heirtzler, H.-L. Dai, S. Jones, J. Zubieta and A. J. Roche, Chem. Commun., 2012, 48, 1000-1002.

313 K. Kamada, C. Hara, K. Ogawa, K. Ohta and Y. Kobuke, Chem. Commun., 2012, 48, 7988-7990.

314 S. Easwaramoorthi, S. Y. Jang, Z. S. Yoon, J. M. Lim, C.-W. Lee, C.-L. Mai, Y.-C. Liu, C.-Y. Yeh, J. Vura-Weis, 
M. R. Wasielewski and D. Kim, J. Phys. Chem. A, 2008, 112, 6563-6570.

315 S. Sreejith and A. Ajayaghosh, Indian J. Chem., Sect. A: Inorg., Bio-inorg., Phys., Theor. Anal. Chem., 2012, 51, 47-56.

316 A. P. de Silva, Molecular logic gates, in Supramolecular Chemistry: From Molecules to Nanomaterials, ed. P. A. Gale and J. W. Steed, 2012, p. 2497.

317 X. Ma and H. Tian, Chem. Soc. Rev., 2010, 39, 70-80.

318 D.-H. Qu, Q.-C. Wang and H. Tian, Angew. Chem., Int. Ed., 2005, 44, 5296-5299.

319 D. A. Leigh, M. Á. F. Morales, E. M. Pérez, J. K. Y. Wong, C. G. Saiz, A. M. Z. Slawin, A. J. Carmichael, D. M. Haddleton, A. M. Brouwer, W. J. Buma, G. W. H. Wurpel, S. León and F. Zerbetto, Angew. Chem., Int. Ed., 2005, 44, 3062-3067.

320 D. H. Qu, F. Y. Ji, Q. C. Wang and H. Tian, Adv. Mater., 2006, 18, 2035-2038.

321 J. E. Green, J. W. Choi, A. Boukai, Y. Bunimovich, E. Johnston-Halperin, E. DeIonno, Y. Luo, B. A. Sheriff, K. Xu, Y. S. Shin, H.-R. Tseng, J. F. Stoddart and J. R. Heath, Nature, 2007, 445, 414-417.

322 M. Feng, L. Gao, Z. Deng, W. Ji, X. Guo, S. Du, D. Shi, D. Zhang, D. Zhu and H. Gao, J. Am. Chem. Soc., 2007, 129, 2204-2205.

323 Z. Dadon, M. Samiappan, N. Wagner, N. Ashkenasy and G. Ashkenasy, Peptide-Based Computation: Switches, Gates and Simple Arithmetic, in Biomolecular Information Processing, ed. E. Katz, Wiley-VCH Verlag \& Co. KGaA, Weinheim, 2012, pp. 9-32.

324 Z. Dadon, M. Samiappan, E. Y. Safranchik and G. Ashkenasy, Chem.-Eur. J., 2010, 16, 12096-12099.

325 M. Samiappan, Z. Dadon and G. Ashkenasy, Chem. Commun., 2011, 47, 710-712.

326 E. Katz and V. Privman, Chem. Soc. Rev., 2010, 39, 18351857.

327 M. Zhou and S. Dong, Biocomputing: Explore Its Realization and Intelligent Logic Detection, in Biomolecular Information Processing, ed. E. Katz, WileyVCH Verlag \& Co. KGaA, Weinheim, 2012, pp. 117-132.

328 I. Willner, B. Shlyahovsky, M. Zayats and B. Willner, Chem. Soc. Rev., 2008, 37, 1153-1165.

329 R. Pei, E. Matamoros, M. Liu, D. Stefanovic and M. N. Stojanovic, Nat. Nanotechnol., 2010, 5, 773-777.

330 G. Seelig, D. Soloveichik, D. Y. Zhang and E. Winfree, Science, 2006, 314, 1585-1588.

331 L. Qian and E. Winfree, Science, 2011, 332, 1196-1201.

332 N. H. Voelcker, K. M. Guckian, A. Saghatelian and M. R. Ghadiri, Small, 2008, 4, 427-431.

333 K. S. Park, M. W. Seo, C. Jung, J. Y. Lee and H. G. Park, Small, 2012, 8, 2203-2212.

334 A. J. Genot, J. Bath and A. J. Turberfield, J. Am. Chem. Soc., 2011, 133, 20080-20083.

335 Y. Xiang, X. Qian, Y. Chen, Y. Zhang, Y. Chai and R. Yuan, Chem. Commun., 2011, 47, 2080-2082.

336 S. J. P. Canete and R. Y. Lai, Chem. Commun., 2010, 46, 3941-3943.

337 K. Schlosser and Y. Li, Chem. Biol., 2009, 16, 311-322.
338 J. Elbaz, O. Lioubashevski, F. Wang, F. Remacle, R. D. Levine and I. Willner, Nat. Nanotechnol., 2010, 5, 417-422.

339 S. Bi, Y. Yan, S. Hao and S. Zhang, Angew. Chem., Int. Ed., 2010, 49, 4438-4442.

340 X. Chen, Y. Wang, Q. Liu, Z. Zhang, C. Fan and L. He, Angew. Chem., Int. Ed., 2006, 45, 1759-1762.

341 T. Li, E. Wang and S. Dong, J. Am. Chem. Soc., 2009, 131, 15082-15083.

342 A. Latorre and Á. Somoza, ChemBioChem, 2012, 13, 951958.

343 T. Li, L. Zhang, J. Ai, S. Dong and E. Wang, ACS Nano, 2011, 5, 6334-6338.

344 H. Pei, L. Liang, G. Yao, J. Li, Q. Huang and C. Fan, Angew.Chem., Int. Ed., 2012, 51, 9020-9024.

345 G. M. Church, Y. Gao and S. Kosuri, Science, 2012, 337, 1628.

346 N. Goldman, P. Bertone, S. Chen, C. Dessimoz, E. M. LeProust, B. Sipos and E. Birney, Nature, 2013, 494, 77-80.

347 Hierarchically Structured Porous Materials: From Nanoscience to Catalysis, Separation, Optics, Energy, and Life Science, ed. B.-L. Su, C. Sanchez and X.-Y. Yang, Wiley-VCH Verlag GmbH \& Co. KGaA, Weinheim, 2011.

348 Applications of Supramolecular Chemistry, ed. H.-J. Schneider, CRC Press, Boca Raton, Florida, 2012.

349 E. García-España, Molecular Recognition, in Supramolecular Chemistry: From Molecules to Nanomaterials, ed. P. Gale and J. Steed, Wiley-V. C. H. Verlag. GmbH \& Co. KGaA, Weinheim, 2012, vol. 3.

350 Metal-Organic Frameworks: Applications from Catalysis to Gas Storage, ed. D. Farrusseng, Wiley-VCH Verlag GmbH \& Co. KGaA, Weinheim, 2011.

351 Functional Metal-Organic Frameworks: Gas Storage, Separation and Catalysis, ed. M. Schröder, Springer, Berlin Heidelberg, 2010, vol. 293.

352 Chemical Sensors and Biosensors: Fundamentals and Applications, ed. F.-G. Banica, Wiley-VCH Verlag $\mathrm{GmbH} \&$ Co. KGaA, Weinheim, 2012.

353 H. Maeda and P. Anzenbacher, Colorimetric Sensors, in Supramolecular Chemistry: From Molecules to Nanomaterials, ed. J. S. P. Gale, Wiley-VCH Verlag GmbH \& Co. KGaA, Weinheim, 2012, vol. 5, p. 2581.

354 M. M. Adams, L. A. Joyce and E. V. Anslyn, Uses of Differential Sensing and Arrays in Chemical Analysis, in Supramolecular Chemistry: From Molecules to Nanomaterials, ed. P. Gale and J. Steed, Wiley-VCH Verlag GmbH \& Co. KGaA, Weinheim, 2012, vol. 2, p. 709.

355 M. Biyikal, M. Hecht, R. Martınez-Manez, K. Rurack and F. Sancenon, Supramolecular Hybrid Nanomaterials as Prospective Sensing Platforms, in Supramolecular Chemistry: From Molecules to Nanomaterials, ed. P. Gale and J. Steed, Wiley-VCH Verlag GmbH \& Co. KGaA, Weinheim, 2012, vol. 8, p. 3669.

356 J. Tian, P. K. Thallapally and B. P. McGrail, Gas Storage and Separation, in Supramolecular Chemistry: From Molecules to Nanomaterials, ed. P. Gale and J. Steed, Wiley-VCH Verlag GmbH \& Co. KGaA, Weinheim, 2012, p. 3133. 
357 S. Matile, N. Sakai and A. Hennig, Transport Experiments in Membranes, in Supramolecular Chemistry: From Molecules to Nanomaterials, ed. P. Gale and J. Steed, Wiley-VCH Verlag GmbH \& Co. KGaA, Weinheim, 2012, vol. 2, p. 473.

358 J. D. Lamb and N. Li, Ion Chromatography and Membrane Separations Using Macrocyclic Ligands, in Supramolecular Chemistry: From Molecules to Nanomaterials, ed. P. Gale and J. Steed, Wiley-VCH Verlag GmbH \& Co. KGaA, Weinheim, 2012, vol. 2, p. 563.

359 G. T. Noble, R. J. Mart, K. Ping Liem and S. J. Webb, Supramolecular Chemistry of Membranes, in Supramolecular Chemistry: From Molecules to Nanomaterials, ed. P. Gale and J. Steed, Wiley-VCH Verlag GmbH \& Co. KGaA, Weinheim, 2012, vol. 4, p. 1731.

360 R. Quesada, Membrane Transport, in Supramolecular Chemistry: From Molecules to Nanomaterials, ed. P. Gale and J. Steed, Wiley-VCH Verlag GmbH \& Co. KGaA, Weinheim, 2012, vol. 4, p. 1751.

361 D. Philp, Supramolecular Reactivity, in Supramolecular Chemistry: From Molecules to Nanomaterials, ed. P. Gale and J. Steed, Wiley-VCH Verlag GmbH \& Co. KGaA, Weinheim, 2012, vol. 4.

362 Supramolecular Catalysis, ed. P. W. N. M. van Leeuwen, Wiley-VCH Verlag GmbH \& Co. KGaA, Weinheim, 2008.

363 Molecular Encapsulation Organic Reactions in Constrained Systems, ed. U. H. Brinker and J.-L. Mieusset, Wiley-VCH Verlag GmbH \& Co. KGaA, Weinheim, 2010.

364 A. Herrmann, Chem.-Eur. J., 2012, 18, 8568-8577.

365 T. Takeuchi and S. Matile, Chem. Commun., 2013, 49, 19-29.

366 L. E. Kreno, K. Leong, O. K. Farha, M. Allendorf, R. P. Van Duyne and J. T. Hupp, Chem.Rev., 2012, 112, 11051125.

367 K. S. Suslick, Curr. Opin. Chem. Biol., 2012, 16, 557-563.

368 G. Aragay, J. Pons and A. Merkoçi, Chem. Rev., 2011, 111, 3433-3458.

369 Y. Song, W. Wei and X. Qu, Adv. Mater., 2011, 23, 42154236.

370 J. S. Fossey, F. D'Hooge, J. M. H. Van Den Elsen, M. P. Pereira Morais, S. I. Pascu, S. D. Bull, F. Marken, A. T. A. Jenkins, Y. B. Jiang and T. D. James, Chem. Rec., 2012, 12, 464-478.

371 C. Han and H. Li, Anal. Bioanal. Chem., 2010, 397, 14371444.

372 H. N. Kim, W. X. Ren, J. S. Kim and J. Yoon, Chem. Soc. Rev., 2012, 41, 3210-3244.

373 I. Yoshimura, Y. Miyahara, N. Kasagi, H. Yamane, A. OjidaItaru and I. Hamachi, J. Am. Chem. Soc., 2004, 126, 12204-12205.

374 A. Wada, S. I. Tamaru, M. Ikeda and I. Hamachi, J. Am. Chem. Soc., 2009, 131, 5321-5330.

375 M. Ikeda, R. Ochi and I. Hamachi, Lab Chip, 2010, 10, 33253334.

376 M. Ikeda, T. Yoshii, T. Matsui, T. Tanida, H. Komatsu and I. Hamachi, J. Am. Chem. Soc., 2011, 133, 1670-1673.

377 H. Komatsu, M. Ikeda and I. Hamachi, Chem. Lett., 2011, 40, 198-200.
378 M. Ikeda, K. Fukuda, T. Tanida, T. Yoshiia and I. Hamachi, Chem. Commun., 2012, 48, 2716-2718.

379 Z. Zhu, C. Wu, H. Liu, Y. Zou, X. Zhang, H. Kang, C. J. Yang and W. Tan, Angew. Chem., Int. Ed., 2010, 49, 10521056.

380 N. Dave, M. Y. Chan, P. J. J. Huang, B. D. Smith and J. Liu, J. Am. Chem. Soc., 2010, 132, 12668-12673.

381 S. Yajima, K. Takami, R. Ooue and K. Kimura, Analyst, 2011, 136, 5131-5133.

382 Y. Xu, L. Zhao, H. Bai, W. Hong, C. Li and G. Shi, J. Am. Chem. Soc., 2009, 131, 13490-13497.

383 Y. Choi, Y. Park, T. Kang and L. P. Lee, Nat. Nanotechnol., 2009, 4, 742-746.

384 C. W. Liu, C. C. Huang and H. T. Chang, Anal. Chem., 2009, 81, 2383-2387.

385 J. S. Lee, M. S. Han and C. A. Mirkin, Angew. Chem., Int. Ed., 2007, 46, 4093-4096.

386 Z. Wang, J. H. Lee and Y. Lu, Adv. Mater., 2008, 20, 32633267.

387 J. Lee, H. Jun and J. Kim, Adv. Mater., 2009, 21, 3674-3677. 388 P. J. Anzenbacher, P. Lubal, P. Bucek, M. A. Palaciosa and M. E. Kozelkova, Chem. Soc. Rev., 2010, 39, 3954-3979.

389 S. H. Lim, L. Feng, J. W. Kemling, C. J. Musto and K. S. Suslick, Nat. Chem., 2009, 1, 562-567.

390 C. J. Musto, S. H. Lim and K. S. Suslick, Anal. Chem., 2009, 81, 6526-6533.

391 J. R. Carey, K. S. Suslick, K. I. Hulkower, J. A. Imlay, K. R. C. Imlay, C. K. Ingison, J. B. Ponder, A. Sen and A. E. Wittrig, J. Am. Chem. Soc., 2011, 133, 7571-7576.

392 P. C. Jurs, G. A. Bakken and H. E. McClelland, Chem. Rev., 2000, 100, 2649-2678.

393 K. Severin, Curr. Opin. Chem. Biol., 2010, 14, 737-742.

394 A. P. Umali and E. V. Anslyn, Curr. Opin. Chem. Biol., 2010, 14, 685-692.

395 A. P. Umali, S. E. LeBoeuf, R. W. Newberry, S. Kim, L. Tran, W. A. Rome, T. Tian, D. Taing, J. Hong, M. Kwan, H. Heymann and E. V. Anslyn, Chem. Sci., 2011, 2, 439-445.

396 B. T. Nguyen and E. V. Anslyn, Coord. Chem. Rev., 2006, 250, 3118-3127.

397 S. Rochat, J. Gao, X. Qian, F. Zaubitzer and K. Severin, Chem.-Eur. J., 2010, 16, 104-113.

398 N. Giuseppone and J. M. Lehn, J. Am. Chem. Soc., 2004, 126, 11448-11449.

399 E. Moulin, G. Cormos and N. Giuseppone, Chem. Soc. Rev., 2012, 41, 1031-1049.

400 S. Otto and K. Severin, Top. Curr. Chem., 2007, 277, 267288

401 S. Rochat and K. Severin, J. Comb. Chem., 2010, 12, 595-599. 402 T. Takeuchi, J. Montenegro, A. Hennig and S. Matile, Chem. Sci., 2011, 2, 303-307.

403 S. M. Butterfield, T. Miyatake and S. Matile, Angew. Chem., Int. Ed., 2009, 48, 325-328.

404 N. Sakai, J. Mareda and S. Matile, Acc. Chem. Res., 2008, 41, 1354-1365.

405 S. Litvinchuk, H. Tanaka, T. Miyatake, D. Pasini, T. Tanaka, G. Bollot, J. Mareda and S. Matile, Nat. Mater., 2007, 6, 576580. 
406 S. Hagihara, H. Tanaka and S. Matile, J. Am. Chem. Soc., 2008, 130, 5656-5657.

407 S. M. Butterfield, D. H. Tran, H. Zhang, G. D. Prestwich and S. Matile, J. Am. Chem. Soc., 2008, 130, 3270-3271.

408 Y. Che, X. Yang, G. Liu, C. Yu, H. Ji, J. Zuo, J. Zhao and L. Zang, J. Am. Chem. Soc., 2010, 132, 5743-5750.

409 Y. N. Wu, F. Li, W. Zhu, J. Cui, C. A. Tao, C. Lin, P. M. Hannam and G. Li, Angew. Chem., Int. Ed., 2011, 50, 12518-12522.

410 Z. Z. Lu, R. Zhang, Y. Z. Li, Z. J. Guo and H. G. Zheng, J. Am. Chem. Soc., 2011, 133, 4172-4174.

411 G. Lu, O. K. Farha, L. E. Kreno, P. M. Schoenecker, K. S. Walton, R. P. Van Duyne and J. T. Hupp, Adv. Mater., 2011, 23, 4449-4452.

412 X. Zou, J. M. Goupil, S. Thomas, F. Zhang, G. Zhu, V. Valtchev and S. Mintova, J. Phys. Chem. C, 2012, 116, 16593-16600.

413 A. L. Robinson, V. Stavila, T. R. Zeitler, M. I. White, S. M. Thornberg, J. A. Greathouse and M. D. Allendorf, Anal. Chem., 2012, 84, 7043-7051.

414 B. Liu, O. Shekhah, H. K. Arslan, J. Liu, C. Wöll and R. A. Fischer, Angew. Chem., Int. Ed., 2012, 51, 807-810.

415 M. M. Wanderley, C. Wang, C.-D. Wu and W. Lin, J. Am. Chem. Soc., 2012, 134, 9050-9053.

416 Y. Takashima, V. Martínez Martínez, S. Furukawa, M. Kondo, S. Shimomura, H. Uehara, M. Nakahama, K. Sugimoto and S. Kitagawa, Nat. Commun., 2011, 2, 168.

417 X. J. Zhao, R. X. He and Y. F. Li, Analyst, 2012, 137, 51905192.

418 M. Dionisio, G. Oliviero, D. Menozzi, S. Federici, R. M. Yebeutchou, F. P. Schmidtchen, E. Dalcanale and P. Bergese, J. Am. Chem. Soc., 2012, 134, 2392-2398.

419 M. Dionisio, J. M. Schnorr, V. K. Michaelis, R. G. Griffin, T. M. Swager and E. Dalcanale, J. Am. Chem. Soc., 2012, 134, 6540-6543.

420 T. H. Kim, H. S. Song, H. J. Jin, S. H. Lee, S. Namgung, U. K. Kim, T. H. Park and S. Hong, Lab Chip, 2011, 11, 2262-2267.

421 D. Bonifazi, S. Mohnani and A. Llanes-Pallas, Chem.-Eur. J., 2009, 15, 7004-7025.

422 A. Ciesielski, C. A. Palma, M. Bonini and P. Samorì, Adv. Mater., 2010, 22, 3506-3520.

423 J. Leclaire, G. Husson, N. Devaux, V. Delorme, L. Charles, F. Ziarelli, P. Desbois, A. Chaumonnot, M. Jacquin, F. Fotiadu and G. Buono, J. Am. Chem. Soc., 2010, 132, 3582-3593.

424 K. Roy, A. C. Wibowo, P. J. Pellechia, S. Ma, M. F. Geer and L. S. Shimizu, Chem. Mater., 2012, 24, 4773-4781.

425 M. B. Dewal, M. W. Lufaso, A. D. Hughes, S. A. Samuel, P. Pellechia and L. S. Shimizu, Chem. Mater., 2006, 18, 4855-4864.

426 N. B. McKeown, J. Mater. Chem., 2010, 20, 10588-10597.

427 J. R. Holst, A. Trewin and A. I. Cooper, Nat. Chem., 2010, 2, 915-920.

428 J. Tian, P. K. Thallapally and B. P. McGrail, CrystEngComm, 2012, 14, 1909-1919.

429 M. Mastalerz, Chem.-Eur. J., 2012, 18, 10082-10091.
430 P. K. Thallapally, B. P. McGrail, J. L. Atwood, C. Gaeta, C. Tedesco and P. Neri, Chem. Mater., 2007, 19, 33553357.

431 C. Tedesco, L. Erra, M. Brunelli, V. Cipolletti, C. Gaeta, A. N. Fitch, J. L. Atwood and P. Neri, Chem.-Eur. J., 2010, 16, 2371-2374.

432 L. Erra, C. Tedesco, V. R. Cipolletti, L. Annunziata, C. Gaeta, M. Brunelli, A. N. Fitch, C. Knöfel, P. L. Llewellyn, J. L. Atwood and P. Neri, Phys. Chem. Chem. Phys., 2012, 14, 311-317.

433 W. Yang, A. Greenaway, X. Lin, R. Matsuda, A. J. Blake, C. Wilson, W. Lewis, P. Hubberstey, S. Kitagawa, N. R. Champness and M. Schröder, J. Am. Chem. Soc., 2010, 132, 14457-14469.

434 C. G. Bezzu, J. E. Warren, M. Helliwell, D. R. Allan and N. B. McKeown, Science, 2010, 327, 1627-1630.

435 Y. He, S. Xiang and B. Chen, J. Am. Chem. Soc., 2011, 133, 14570-14573.

436 M. Mastalerz and I. M. Oppel, Angew. Chem., Int. Ed., 2012, 51, 5252-5255.

437 T. Tozawa, J. T. A. Jones, S. I. Swamy, S. Jiang, D. J. Adams, S. Shakespeare, R. Clowes, D. Bradshaw, T. Hasell, S. Y. Chong, C. Tang, S. Thompson, J. Parker, A. Trewin, J. Bacsa, A. M. Z. Slawin, A. Steiner and A. I. Cooper, Nat. Mater., 2009, 8, 973-978.

438 J. T. A. Jones, D. Holden, T. Mitra, T. Hasell, D. J. Adams, K. E. Jelfs, A. Trewin, D. J. Willock, G. M. Day, J. Bacsa, A. Steiner and A. I. Cooper, Angew. Chem., Int. Ed., 2011, 50, 749-753.

439 J. T. A. Jones, T. Hasell, X. F. Wu, J. Bacsa, K. E. Jelfs, M. Schmidtmann, S. Y. Chong, D. J. Adams, A. Trewin, F. Schiffman, F. Cora, B. Slater, A. Steiner, G. M. Day and A. I. Cooper, Nature, 2011, 474, 367-371.

440 T. Hasell, S. Y. Chong, K. E. Jelfs, D. J. Adams and A. I. Cooper, J. Am. Chem. Soc., 2012, 134, 588-598.

441 T. Hasell, H. Zhang and A. I. Cooper, Adv. Mater., 2012, 24, 5732-5737.

442 M. Mastalerz, M. W. Schneider, I. M. Oppel and O. Presly, Angew. Chem., Int. Ed., 2011, 50, 1046-1051.

443 M. W. Schneider, I. M. Oppel, H. Ott, L. G. Lechner, H. J. S. Hauswald, R. Stoll and M. Mastalerz, Chem.-Eur. J., 2012, 18, 836-847.

444 M. Brutschy, M. W. Schneider, M. Mastalerz and S. R. Waldvogel, Adv. Mater., 2012, 24, 6049-6052.

445 R. Afonso, A. Mendes and L. Gales, J. Mater. Chem., 2012, 22, 1709-1723.

446 R. V. Afonso, J. Durão, A. Mendes, A. M. Damas and L. Gales, Angew. Chem., Int. Ed., 2010, 49, 3034-3036.

447 S. Maity, P. Jana and D. Haldar, CrystEngComm, 2011, 13, 3064-3071.

448 J. Rabone, Y. F. Yue, S. Chong, K. Stylianou, J. Bacsa, D. Bradshaw, G. Darling, N. Berry, Y. Khimyak, A. Ganin, P. Wiper, J. B. Claridge and M. J. Rosseinsky, Science, 2010, 329, 1053-1057.

449 C. Martí-Gastaldo, J. E. Warren, K. C. Stylianou, N. L. O. Flack and M. J. Rosseinsky, Angew. Chem., Int. Ed., 2012, 51, 11044-11048. 
450 H. Furukawa, N. Ko, Y. B. Go, N. Aratani, S. B. Choi, E. Choi, A. O. Yazaydin, R. Q. Snurr, M. O'Keeffe, J. Kim and O. M. Yaghi, Science, 2010, 329, 424-428.

451 O. K. Farha, A. O. Yazaydin, I. Eryazici, C. D. Malliakas, B. G. Hauser, M. G. Kanatzidis, S. T. Nguyen, R. Q. Snurr and J. T. Hupp, Nat. Chem., 2010, 2, 944-948.

452 O. K. Farha, I. Eryazici, N. C. Jeong, B. G. Hauser, C. E. Wilmer, A. A. Sarjeant, R. Q. Snurr, S. B. T. Nguyen, A. O. Yazaydin and J. T. Hupp, J. Am. Chem. Soc., 2012, 134, 15016-15021.

453 T. A. Makal, J. R. Li, W. Lu and H. C. Zhou, Chem. Soc. Rev., 2012, 41, 7761-7779.

454 M. P. Suh, H. J. Park, T. K. Prasad and D. W. Lim, Chem. Rev., 2012, 112, 782-835.

455 J. R. Li, R. J. Kuppler and H. C. Zhou, Chem. Soc. Rev., 2009, 38, 1477-1504.

456 K. Sumida, D. L. Rogow, J. A. Mason, T. M. McDonald, E. D. Bloch, Z. R. Herm, T. H. Bae and J. R. Long, Chem. Rev., 2012, 112, 724-781.

457 J. Liu, P. K. Thallapally, B. P. McGrail, D. R. Brown and J. Liu, Chem. Soc. Rev., 2012, 41, 2308-2322.

458 T. M. McDonald, W. R. Lee, J. A. Mason, B. M. Wiers, C. S. Hong and J. R. Long, J. Am. Chem. Soc., 2012, 134, 7056-7065.

459 S. Yang, X. Lin, W. Lewis, M. Suyetin, E. Bichoutskaia, J. E. Parker, C. C. Tang, D. R. Allan, P. J. Rizkallah, P. Hubberstey, N. R. Champness, K. M. Thomas, A. J. Blake and M. Schröder, Nat. Mater., 2012, 11, 710-716. 460 J. P. Zhang and X. M. Chen, J. Am. Chem. Soc., 2008, 130, 6010-6017.

461 S. Shimomura, M. Higuchi, R. Matsuda, K. Yoneda, Y. Hijikata, Y. Kubota, Y. Mita, J. Kim, M. Takata and S. Kitagawa, Nat. Chem., 2010, 2, 633-637.

462 N. Yanai, K. Kitayama, Y. Hijikata, H. Sato, R. Matsuda, Y. Kubota, M. Takata, M. Mizuno, T. Uemura and S. Kitagawa, Nat. Mater., 2011, 10, 787-793.

463 S. Matile, A. Vargas Jentzsch, J. Montenegro and A. Fin, Chem. Soc. Rev., 2011, 40, 2453-2474.

464 J. K. W. Chui and T. M. Fyles, Chem. Soc. Rev., 2012, 41, 148-175.

465 P. R. Brotherhood and A. P. Davis, Chem. Soc. Rev., 2010, 39, 3633-3647.

466 H. Cho, L. Widanapathirana and Y. Zhao, J. Am. Chem. Soc., 2011, 133, 141-147.

467 W. Si, L. Chen, X. B. Hu, G. Tang, J. L. Hou and Z. T. Li, Angew. Chem., Int. Ed., 2011, 50, 12564-12568.

468 X. B. Hu, L. Chen, G. Tang, J. L. Hou and Z. T. Li, J. Am. Chem. Soc., 2012, 134, 8384-8387.

469 T. Kiwada, K. Sonomura, Y. Sugiura, K. Asami and S. Futaki, J. Am. Chem. Soc., 2006, 128, 6010-6011.

470 A. Satake, M. Yamamura, M. Oda and Y. Kobuke, J. Am. Chem. Soc., 2008, 130, 6314-6315.

471 C. P. Wilson and S. J. Webb, Chem. Commun., 2008, 40074009.

472 C. P. Wilson, C. Boglio, L. Ma, S. L. Cockroft and S. J. Webb, Chem.-Eur. J., 2011, 17, 3465-3473.

473 S. Bhosale, A. L. Sisson, P. Talukdar, A. Fürstenberg, N. Banerji, E. Vauthey, G. Bollot, J. Mareda, C. Röger, F. Würthner, N. Sakai and S. Matile, Science, 2006, 313, 84-86.
474 P. Talukdar, G. Bollot, J. Mareda, N. Sakai and S. Matile, J. Am. Chem. Soc., 2005, 127, 6528-6529.

475 A. V. Jentzsch, A. Hennig, J. Mareda and S. Matile, Acc. Chem. Res., 2013, 46, DOI: 10.1021/ar400014r.

476 A. Hennig, L. Fischer, G. Guichard and S. Matile, J. Am. Chem. Soc., 2009, 131, 16889-16895.

477 R. E. Dawson, A. Hennig, D. P. Weimann, D. Emery, V. Ravikumar, J. Montenegro, S. G. T. Takeuchi, M. Mayor, J. Mareda, C. A. Schalley and S. Matile, Nat. Chem., 2010, 2, 533-538.

478 J. Mareda and S. Matile, Chem.-Eur. J., 2009, 15, 28-37.

479 A. V. Jentzsch, D. Emery, J. Mareda, S. K. Nayak, P. Metrangolo, G. Resnati, N. Sakai and S. Matile, Nat. Commun., 2012, 3, 905.

480 E. Krieg and B. Rybtchinski, Chem.-Eur. J., 2011, 17, 90169026.

481 M. Barboiu, Chem. Commun., 2010, 46, 7466-7476.

482 Y. Lu, T. Suzuki, W. Zhang, J. S. Moore and B. J. Mariñas, Chem. Mater., 2007, 19, 3194-3204.

483 X. Peng, J. Jin, Y. Nakamura, T. Ohno and I. Ichinose, Nat. Nanotechnol., 2009, 4, 353-357.

484 E. Krieg, H. Weissman, E. Shirman, E. Shimoni and B. Rybtchinski, Nat. Nanotechnol., 2011, 6, 141-146.

485 P. Tyagi, A. Deratani, D. Bouyer, D. Cot, V. Gence, M. Barboiu, T. N. T. Phan, D. Bertin, D. Gigmes and D. Quemener, Angew. Chem., Int. Ed., 2012, 51, 7166-7170.

486 A. Cazacu, Y. M. Legrand, A. Pasc, G. Nasr, A. Van der Lee, E. Mahon and M. Barboiu, Proc. Natl. Acad. Sci. U. S. A., 2009, 106, 8117-8122.

487 Y. Le Duc, M. Michau, A. Gilles, V. Gence, Y. M. Legrand, A. van der Lee, S. Tingry and M. Barboiu, Angew. Chem., Int. Ed., 2011, 50, 11366-11372.

488 G. Nasr, T. Macron, A. Gilles, Z. Mouline and M. Barboiu, Chem. Commun., 2012, 48, 6827-6829.

489 G. Nasr, T. Macron, A. Gilles, E. Petit and M. Barboiu, Chem. Commun., 2012, 48, 7398-7400.

490 M. Barboiu and A. Gilles, Acc. Chem. Res., 2013, 46, DOI: 10.1021/ar400025e.

491 M. S. Kaucher, M. Peterca, A. E. Dulcey, A. J. Kim, S. A. Vinogradov, D. A. Hammer, P. A. Heiney and V. Percec, J. Am. Chem. Soc., 2007, 129, 11698-11699.

492 J. R. Li, J. Sculley and H. C. Zhou, Chem. Rev., 2012, 112, 869-932.

493 B. Liu, J. Mater. Chem., 2012, 22, 10094-10101.

494 L. Alaerts, C. E. A. Kirschhock, M. Maes, M. A. van der Veen, V. Finsy, A. Depla, J. A. Martens, G. V. Baron, P. A. Jacobs, J. F. M. Denayer and D. E. De Vos, Angew. Chem., Int. Ed., 2007, 46, 4293-4297.

495 M. Maes, F. Vermoortele, L. Alaerts, S. Couck, C. E. A. Kirschhock, J. F. M. Denayer and D. E. De Vos, J. Am. Chem. Soc., 2010, 132, 15277-15285.

496 Z. Y. Gu and X. P. Yan, Angew. Chem., Int. Ed., 2010, 49, 1477-1480.

497 N. Chang, Z. Y. Gu and X. P. Yan, J. Am. Chem. Soc., 2010, 132, 13645-13647.

498 A. S. Münch, J. Seidel, A. Obst, E. Weber and F. O. R. L. Mertens, Chem.-Eur. J., 2011, 17, 10958-10964. 
499 A. L. Nuzhdin, D. N. Dybtsev, K. P. Bryliakov, E. P. Talsi and V. P. Fedin, J. Am. Chem. Soc., 2007, 129, 12958-12959.

500 S. Han, Y. Wei, C. Valente, I. Lagzi, J. J. Gassensmith, A. Coskun, J. F. Stoddart and B. A. Grzybowski, J. Am. Chem. Soc., 2010, 132, 16358-16361.

501 Y. S. Li, F. Y. Liang, H. Bux, A. Feldhoff, W. S. Yang and J. Caro, Angew. Chem., Int. Ed., 2010, 49, 548-551.

502 M. J. C. Ordoñez, K. J. Balkus, Jr, J. P. Ferraris and I. H. Musselman, J. Membr. Sci., 2010, 361, 28-37.

503 T. Ema, J. Inclusion Phenom. Macrocyclic Chem., 2012, 74, 41-55.

504 E. M. Pérez and N. Martín, Org. Biomol. Chem., 2012, 10, 3577-3583.

505 N. Komatsu and F. Wang, Materials, 2010, 3, 3818-3844.

506 X. Peng, N. Komatsu, S. Bhattacharya, T. Shimawaki, S. Aonuma, T. Kimura and A. Osuka, Nat. Nanotechnol., 2007, 2, 361-365.

507 X. Tu, S. Manohar, A. Jagota and M. Zheng, Nature, 2009, 460, 250-253.

508 Y. Shoji, K. Tashiro and T. Aida, J. Am. Chem. Soc., 2010, 132, 5928-5929.

509 T. Ema, K. Hamada, K. Sugita, Y. Nagata, T. Sakai and A. Ohnishi, J. Org. Chem., 2010, 75, 4492-4500.

510 T. R. Jackson, J. S. Springall, D. Rogalle, N. Masumoto, H. C. Li, F. D'Hooge, S. P. Perera, A. T. A. Jenkins, T. D. James, J. S. Fossey and J. M. H. van den Elsen, Electrophoresis, 2008, 29, 4185-4191.

511 M. P. Pereira Morais, J. D. Mackay, S. K. Bhamra, J. G. Buchanan, T. D. James, J. S. Fossey and J. M. van den Elsen, Proteomics, 2010, 10, 48-58.

512 M. J. Wiester, P. A. Ulmann and C. A. Mirkin, Angew. Chem., Int. Ed., 2011, 50, 114-137.

513 Y. Yin, Z. Dong, Q. Luo and J. Liu, Prog. Polym. Sci., 2012, 37, 1476-1509.

514 Z. Dong, Q. Luo and J. Liu, Chem. Soc. Rev., 2012, 41, 78907908.

515 H. Amouri, C. Desmarets and J. Moussa, Chem. Rev., 2012, 112, 2015-2041.

516 K. T. Kim, S. A. Meeuwissen, R. J. M. Nolte and J. C. M. van Hest, Nanoscale, 2010, 2, 844-858.

517 M. Yoshizawa and M. Fujita, Bull. Chem. Soc. Jpn., 2010, 83, 609-618.

518 M. Yoshizawa, M. Tamura and M. Fujita, Science, 2006, 312, 251-254.

519 T. Murase, Y. Nishijima and M. Fujita, J. Am. Chem. Soc., 2012, 134, 162-164.

520 M. D. Pluth, R. G. Bergman and K. N. Raymond, Science, 2007, 316, 85-88.

521 C. J. Hastings, M. P. Backlund, R. G. Bergman and K. N. Raymond, Angew. Chem., Int. Ed., 2011, 50,10570-10573.

522 W. M. Hart-Cooper, K. N. Clary, F. D. Toste, R. G. Bergman and K. N. Raymond, J. Am. Chem. Soc., 2012, 134, 1787317876.

523 Z. J. Wang, K. N. Clary, R. G. Bergman, K. N. Raymond and F. D. Toste, Nat. Chem., 2013, 5, 100-103.

524 N. Sakai, N. Sordé and S. Matile, J. Am. Chem. Soc., 2003, 125, 7776-7777.
525 S. Litvinchuk, G. Bollot, J. Mareda, A. Som, D. Ronan, M. Raza Shah, P. Perrottet, N. Sakai and S. Matile, J. Am. Chem. Soc., 2004, 126, 10067-10075.

526 M. O. Guler and S. I. Stupp, J. Am. Chem. Soc., 2007, 129, 12082-12083.

527 D. Zaramella, P. Scrimin and L. J. Prins, J. Am. Chem. Soc., 2012, 134, 8396-8399.

528 J. Yang, M. B. Dewal, S. J. Profeta, M. D. Smith, Y. Li and L. S. Shimizu, J. Am. Chem. Soc., 2008, 130, 612-621.

529 S. Dawn, M. B. Dewal, D. Sobransingh, M. C. Paderes, A. C. Wibowo, M. D. Smith, J. A. Krause, P. J. Pellechia and L. S. Shimizu, J. Am. Chem. Soc., 2011, 133, 7025-7032. 530 C. H. Görbitz, Chem.-Eur. J., 2007, 13, 1022-1031.

531 G. Distefano, A. Comotti, S. Bracco, M. Beretta and P. Sozzani, Angew. Chem., Int. Ed., 2012, 51, 9258-9262.

532 B. Escuder, F. Rodríguez-Llansola and J. F. Miravet, New J. Chem., 2010, 34, 1044-1054.

533 F. Rodríguez-Llansola, B. Escuder and J. F. Miravet, J. Am. Chem. Soc., 2009, 131, 11478-11484.

534 Y. R. Liu, L. He, J. Zhang, X. Wang and C. Y. Su, Chem. Mater., 2009, 21, 557-563.

535 L. Ma and W. Lin, Top. Curr. Chem., 2010, 293, 175-205.

536 A. Corma, H. García and F. X. Llabrés i Xamena, Chem. Rev., 2010, 110, 4606-4655.

537 M. Yoon, R. Srirambalaji and K. Kim, Chem. Rev., 2012, 112, 1196-1231.

538 D.-L. Long, R. Tsunashima and L. Cronin, Angew. Chem., Int. Ed., 2010, 49, 1736-1758.

539 J. M. Falkowski, C. Wang, S. Liu and W. Lin, Angew. Chem., Int. Ed., 2011, 50, 8674-8678.

540 C. Zhu, G. Yuan, X. Chen, Z. Yang and Y. Cui, J. Am. Chem. Soc., 2012, 134, 8058-8061.

541 P. Wu, C. He, J. Wang, X. Peng, X. Li, Y. An and C. Duan, J. Am. Chem. Soc., 2012, 134, 14991-14999.

542 M. Banerjee, S. Das, M. Yoon, H. J. Choi, M. H. Hyun, S. M. Park, G. Seo and K. Kim, J. Am. Chem. Soc., 2009, 132, 7524-7525.

543 L. Ma, J. M. Falkowski, C. Abney and W. Lin, Nat. Chem., 2010, 2, 838-846.

544 A. Dhakshinamoorthy and H. Garcia, Chem. Soc. Rev., 2012, 41, 5262-5284.

545 G. Q. Kong, S. Ou, C. Zou and C. D. Wu, J. Am. Chem. Soc., 2012, 134, 19851-19857.

546 D. Feng, Z. Y. Gu, J. R. Li, H. L. Jiang, Z. Wei and H. C. Zhou, Angew. Chem., Int.Ed., 2012, 51, 10307-10310.

547 S. Förster and T. Plantenberg, Angew. Chem., Int. Ed., 2002, 41, 688-714.

548 D. M. Vriezema, P. M. L. Garcia, N. S. Oltra, N. S. Hatzakis, S. M. Kuiper, R. J. M. Nolte, A. E. Rowan and J. C. M. van Hest, Angew. Chem., Int. Ed., 2007, 46, 7378-7382.

549 Z. Wang, M. C. M. van Oers, F. P. J. T. Rutjes and J. C. M. van Hest, Angew. Chem., Int. Ed., 2012, 51, 10746-10750.

550 E. Moulin and N. Giuseppone, Reactions in Dynamic SelfAssemblies, in Supramolecular Chemistry: From Molecules to Nanomaterials, ed. P. Gale and J. Steed, Wiley-VCH Verlag GmbH \& Co. KGaA, Weinheim, 2012, vol. 4, pp. 1543-1574. 
551 S. R. K. Minkler, B. H. Lipshutz and N. Krause, Angew. Chem., Int. Ed., 2011, 50, 7820-7823.

552 B. H. Lipshutz, S. Huang, W. W. Y. Leong, G. Zhong and N. A. Isley, J. Am. Chem. Soc., 2012, 134, 19985-19988.

553 J. Li, Y. Tang, Q. Wang, X. Li, L. Cun, X. Zhang, J. Zhu, L. Li and J. Deng, J. Am. Chem. Soc., 2012, 134, 1852218525.

554 R. McHale, J. P. Patterson, P. B. Zetterlund and R. K. O'Reilly, Nat. Chem., 2012, 4, 491-497.

555 M. M. Müller, M. A. Windsor, W. C. Pomerantz, S. H. Gellman and D. Hilvert, Angew. Chem., Int. Ed., 2009, 48, 922-925.
556 T. Terashima, T. Mes, T. F. A. De Greef, M. A. J. Gillissen, P. Besenius, A. R. A. Palmans and E. W. Meijer, J. Am. Chem. Soc., 2011, 133, 4742-4745.

557 E. Huerta, P. J. M. Stals, E. W. Meijer and A. R. A. Palmans, Angew. Chem., Int. Ed., 2013, 52, 2906-2910.

558 S. Cantekin, T. F. A. de Greef and A. R. A. Palmans, Chem. Soc. Rev., 2012, 41, 6125-6137.

559 O. I. Wilner, Y. Weizmann, R. Gill, O. Lioubashevski, R. Freeman and I. Willner, Nat. Nanotechnol., 2009, 4, 249-254.

560 S. Frühbeißer and F. Grohn, J. Am. Chem. Soc., 2012, 134, 14267-14270. 